\title{
Use of the Nosyl Group as a Functional Protecting Group in Applications of a Michael/Smiles Tandem Process
}

\author{
Siomenan Coulibali, Timothé Godou and Sylvain Canesi* \\ Laboratoire de Méthodologie et Synthèse de Produit Naturels. \\ Université du Québec à Montréal, C.P.8888, Succ. Centre-Ville, Montréal. H3C 3P8, \\ Québec, Canada.
}

\section{Supporting Information}

\section{Table of Contents}

1. General information

$-\mathrm{S} 1-$

2. Experimental procedures and descriptions

$-\mathrm{S} 2-\mathrm{S} 12$

3. Copies of ${ }^{1} \mathrm{H}$ and ${ }^{13} \mathrm{C}$ NMR spectra for all compounds

\section{General information and materials}

Unless otherwise indicated, ${ }^{1} \mathrm{H}$ and ${ }^{13} \mathrm{C}$ NMR spectra were recorded at 300 and $75 \mathrm{MHz}$, respectively, in $\mathrm{CDCl}_{3}$ solutions. Chemical shifts are reported in ppm on the $\delta$ scale. Multiplicities are described as s (singlet), d (doublet), dd, ddd, etc. (doublet of doublets, doublet of doublets of doublets, etc.), t (triplet), q (quartet), quin (quintuplet), m (multiplet), and further qualified as app (apparent), br (broad). Coupling constants, J, are reported in Hz. IR spectra (cm-1) were recorded from thin films. HRMS were measured in the electrospray (ESI) mode on a LC-MSD TOF mass analyzer. 


\section{General Procedure for the Formation of dienone 8 or dienimine 21.}

To a stirred solution of phenols or sulphonamides $(0.5 \mathrm{mmol})$ in methanol $(5 \mathrm{~mL})$ at $20^{\circ} \mathrm{C}$ was added $\mathrm{PhI}(\mathrm{OAc}) 2$ ("DIB" $0.6 \mathrm{mmol}, 1.2$ equiv) dissolved in methanol $(2 \mathrm{~mL})$, over $10 \mathrm{~s}$. The reaction was stirred for $10 \mathrm{~min}$, concentrated then purified by chromatography (hexane:ethyl acetate as required or ethyl acetate- $\mathrm{MeOH}(95 / 5)$ for compound $\mathbf{8 i}$.

General Procedure for the Formation of enone 12. To a solution of dienone $8(0.2 \mathrm{mmol}$, 1.0 equiv) in Acetonitrile $(2.0 \mathrm{~mL})$ was added Cesium carbonate ( $0.6 \mathrm{mmol}, 3.0$ equiv), and the solution was stirred at $65{ }^{\circ} \mathrm{C}$. The reaction was followed by TLC. After completion, the reaction mixture was quenched with water and extracted with ethyl acetate $(3 \times 50 \mathrm{~mL})$. The organic layer was separated, dried with $\mathrm{Na}_{2} \mathrm{SO}_{4}$, and concentrated under reduced pressure. The residue was then purified by silica gel chromatography (on a short pad) with a mixture of ethyl acetate/hexane (or ethyle acetate-MeOH (3/1) for compound 12i) to give the corresponding enone $\mathbf{1 2}$ as a yellow oil.

General Procedure for the Formation of ketones 15, 17 et 20. To a solution of cyclohexenone 13 (0.5 mmol, 1.0 equiv) in Acetonitrile $(3.0 \mathrm{~mL})$ was added methyl 2-(2nitrophenylsulfonyl) acetate 16 (1. $02 \mathrm{mmol}, 2$ equiv) and cesium carbonate (1.5 mmol, 3.0 equiv). The solution was stirred at $80^{\circ} \mathrm{C}$ over night. After completion, the mixture was directly filtrated on a small pad of silica gel (washed with ethyl acetate) and then concentrated under reduced pressure. The crude product was purified by chromatography. (n-hexane/ethyl acetate) to afford the corresponding ketone 17. The same procedure with methyl 2-(4nitrophenylsulfonyl) acetate $\mathbf{1 4}$ leads to ketone $\mathbf{1 5}$ in a similar yield.

\section{General procedure for the formation of compound 25}

To a stirred solution of compound $23(50 \mathrm{mg}, 0.3 \mathrm{mmol})$ in dichloromethane $(2 \mathrm{~mL})$, was added a few drops of TFA. The solution was stirred at room temperature until the reaction was completed as indicated by TLC. The reaction mixture was concentrated under reduced pressure and purified by flash chromatography over silica.

Compound 30: To a solution of $\mathbf{2 6}(77.6 \mathrm{mg}, 0.2 \mathrm{mmol}, 1.0$ equiv) in acetonitrile $(2.0 \mathrm{~mL})$ was added dimethylmalonate $(79.2 \mathrm{mg}, 0.6 \mathrm{mmol}, 3.0$ equiv) and cesium carbonate $(98 \mathrm{mg}$, 
$0.6 \mathrm{mmol}, 3.0$ equiv), the solution was stirred at $82^{\circ} \mathrm{C}$ overnight. After completion, the reaction mixture was quenched with $\mathrm{NH}_{4} \mathrm{Cl}$ and extracted with ethyl acetate $(3 \times 50 \mathrm{~mL})$. The organic layer was separated, dried with $\mathrm{Na}_{2} \mathrm{SO}_{4}$, and concentrated under reduced pressure. The residue was then purified by silica gel chromatography with a mixture of ethyl acetate/hexane (22:78) to give a diasteromeric mixture of compound $\mathbf{3 0}(51 \mathrm{mg}, 63 \%)$ that was directly used fort he formation of indole $\mathbf{3 3}$ by reduction on hydrogene (see general procedure below).

General Procedure for the Formation of indole 30, 31 and 33. To a solution of ketones 12, 17 or 32 (0.075 mmol, 1 equiv) in $\mathrm{MeOH}(2 \mathrm{ml})$ was added $\mathrm{Pd}-\mathrm{C}(0.0075 \mathrm{mmol}, 0.1$ equiv). The mixture was stirred at room temperature and the reaction was followed by TLC. After completion the mixture was filtered through Celite and concentrated under vacuum. The residue was purified by silica gel chromatography with a mixture of ethyl acetate /hexane to afford the corresponding indole.

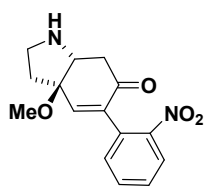

1,2,3,3a,7,7a-hexahydro-3a-methoxy-5-(2-nitrophenyl)indol-6-one (12a): (52 mg, 90\% yield). ${ }^{1} \mathrm{H}$ NMR (300 MHz, $\left.\mathrm{CDCl}_{3}\right) \delta 8.08$ (d, $\left.J=8.1 \mathrm{~Hz}, 1 \mathrm{H}\right), 7.64$ (t, $J=8.1 \mathrm{~Hz}$, 1H), 7.52 (t, $J=8.1 \mathrm{~Hz}, 1 \mathrm{H}), 7.29(\mathrm{~d}, J=8.1 \mathrm{~Hz}, 1 \mathrm{H}), 6.83(\mathrm{~s}, 1 \mathrm{H}), 3.85$ (dd, $J=7.8,5.8 \mathrm{~Hz}$, $1 \mathrm{H}), 3.40(\mathrm{~s}, 3 \mathrm{H}), 3.22(\mathrm{~m}, 2 \mathrm{H}), 2.87(\mathrm{dd}, J=16.2,5.6 \mathrm{~Hz}, 1 \mathrm{H}), 2.66(\mathrm{dd}, 16.2,8.0 \mathrm{~Hz}, 1 \mathrm{H})$, $2.20(\mathrm{~m}, 2 \mathrm{H}) ;{ }^{13} \mathrm{C}$ NMR $\left(75 \mathrm{MHz}, \mathrm{CDCl}_{3}\right) \delta 195.1,148.1,144.7,140.4,133.6,131.6,131.2$, 129.4, 124.5, 83.0, 60.1, 52.2, 44.6, 42.5, 38.3; HRMS (ESI): Calc. for $\mathrm{C}_{15} \mathrm{H}_{17} \mathrm{~N}_{2} \mathrm{O}_{4}(\mathrm{M}+\mathrm{H})^{+}$: 289.1183; found: 289.1188 .

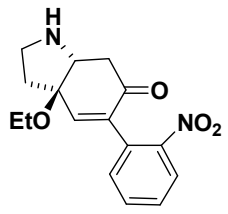

(3aR,7aR)-3a-ethoxy-1,2,3,3a,7,7a-hexahydro-5-(2-nitrophenyl)indol-6-one (12b): (51 mg, 86\% yield). ${ }^{1} \mathrm{H}$ NMR $\left(300 \mathrm{MHz}, \mathrm{CDCl}_{3}\right) \delta 8.07(\mathrm{~d}, J=8.1 \mathrm{~Hz}, 1 \mathrm{H}), 7.63(\mathrm{t}, J$ $=8.1 \mathrm{~Hz}, 1 \mathrm{H}), 7.52(\mathrm{t}, J=8.1 \mathrm{~Hz}, 1 \mathrm{H}), 7.29(\mathrm{~d}, J=8.1 \mathrm{~Hz}, 1 \mathrm{H}), 6.83(\mathrm{~s}, 1 \mathrm{H}), 3.85(\mathrm{dd}, J=$ 7.8, $5.8 \mathrm{~Hz}, 1 \mathrm{H}), 3.61(\mathrm{~m}, 2 \mathrm{H}), 3.22(\mathrm{~m}, 2 \mathrm{H}), 2.87$ (dd, $J=16.2,5.6 \mathrm{~Hz}, 1 \mathrm{H}), 2.67$ (dd, 16.2, $8.0 \mathrm{~Hz}, 1 \mathrm{H}), 2.21(\mathrm{~m}, 2 \mathrm{H}), 1.24(\mathrm{t}, 8.0 \mathrm{~Hz}) ;{ }^{13} \mathrm{C} \mathrm{NMR}\left(75 \mathrm{MHz}, \mathrm{CDCl}_{3}\right) \delta 195.2,148.1,145.4$, 
133.5, 131.6, 131.2, 129.3, 124.4, 82.6, 60.6, 60.0, 44.5, 42.3, 38.6, 16.0; HRMS (ESI): Calc. for $\mathrm{C}_{16} \mathrm{H}_{19} \mathrm{~N}_{2} \mathrm{O}_{4}(\mathrm{M}+\mathrm{H})^{+}:$303.1339; found: 303.1337 .



1,2,3,3a,7,7a-hexahydro-3a-methoxy-5-(4-nitrophenyl)indol-6-one (12c): (47 mg, 82\% yield). ${ }^{1} \mathrm{H}$ NMR (300 MHz, $\left.\mathrm{CDCl}_{3}\right) \delta 8.22(\mathrm{~d}, J=8.7 \mathrm{~Hz}, 2 \mathrm{H}), 7.55(\mathrm{~d}, J=8.7$ Hz, 2H), $7.28(\mathrm{~s}, 1 \mathrm{H}), 3.97-3.78(\mathrm{~m}, 1 \mathrm{H}), 3.38(\mathrm{~s}, 3 \mathrm{H}), 3.34-3.11(\mathrm{~m}, 2 \mathrm{H}), 2.92$ (dd, $J=$ 16.2, $5.5 \mathrm{~Hz}, 1 \mathrm{H}), 2.68(\mathrm{dd}, J=16.2,8.4 \mathrm{~Hz}, 1 \mathrm{H}), 2.46-2.07(\mathrm{~m}, 2 \mathrm{H}) .{ }^{13} \mathrm{C}$ NMR $(75 \mathrm{MHz}$, $\left.\mathrm{CDCl}_{3}\right) \delta 195.5,148.1,147.3,141.3,138.7,129.2,123.1,82.9,59.9,51.8,44.4,42.9,37.7$, 29.4. HRMS (ESI): Calc. for $\mathrm{C}_{15} \mathrm{H}_{17} \mathrm{~N}_{2} \mathrm{O}_{4}(\mathrm{M}+\mathrm{H})^{+}$: 289,1183; found: 289,1179 .

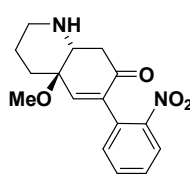

\section{2,3,4,4a,8,8a-hexahydro-4a-methoxy-6-(2-nitrophenyl)quinolin-7(1H)-one}

(12d): (56 mg, 94\% yield). ${ }^{1} \mathrm{H}$ NMR (300 MHz, $\left.\mathrm{CDCl}_{3}\right) \delta 8.08$ (dd, $\left.J=8.1,1.2 \mathrm{~Hz}, 1 \mathrm{H}\right), 7.64$ $(\mathrm{td}, J=8.1,1.2 \mathrm{~Hz}, 1 \mathrm{H}), 7.52(\mathrm{td}, J=8.1,1.2 \mathrm{~Hz}, 1 \mathrm{H}), 7.30(\mathrm{~d}, J=8.1,1.2 \mathrm{~Hz}, 1 \mathrm{H}), 6.74(\mathrm{~s}$, $1 \mathrm{H}), 3.54(\mathrm{dd}, J=8.5,4.5 \mathrm{~Hz}, 1 \mathrm{H}), 3.40(\mathrm{~s}, 3 \mathrm{H}), 3.02(\mathrm{dd}, J=16.7,4.4 \mathrm{~Hz}, 2 \mathrm{H}), 2.95$ (m, 1H), 2. 83 (m, 1H), 2.77 (dd, $J=16.7,8.5 \mathrm{~Hz}, 1 \mathrm{H}), 2.30$ (br, 1H), 2.09 (m, 1H), 1.83 (m, 2H), $1.51(\mathrm{~m}, 1 \mathrm{H}) ;{ }^{13} \mathrm{C} \mathrm{NMR}\left(75 \mathrm{MHz}, \mathrm{CDCl}_{3}\right) \delta 195.2,148.3,147.0,141.1,133.5,131.6$, 131.2, 129.3, 124.4, 72.7, 50.4, 41.9, 40.5, 31.2, 21.9; HRMS (ESI): Calc. for $\mathrm{C}_{16} \mathrm{H}_{19} \mathrm{~N}_{2} \mathrm{O}_{4}$ $(\mathrm{M}+\mathrm{H})^{+}:$303.1339; found: 303.1344 .

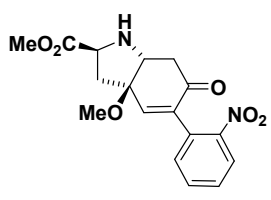

\section{Methyl 2,3,3a,6,7,7a-hexahydro-3a-methoxy-5-(2-nitrophenyl)-6-oxo-}

1H-indole-2-carboxylate (12e): (43 mg, 62\% yield). ${ }^{1} \mathrm{H} \mathrm{NMR}\left(300 \mathrm{MHz}, \mathrm{CDCl}_{3}\right) \delta 8.10$ (dd, $J=8.1,1.2 \mathrm{~Hz}, 1 \mathrm{H}), 7.65(\mathrm{td}, J=8.1,1.2 \mathrm{~Hz}, 1 \mathrm{H}), 7.53(\mathrm{td}, J=8.1,1.2 \mathrm{~Hz}, 1 \mathrm{H}), 7.29(\mathrm{~d}, J=$ 8.1, 1.2 Hz, 1H), $6.74(\mathrm{~s}, 1 \mathrm{H}), 4.14(\mathrm{t}, J=8.1 \mathrm{~Hz}, 1 \mathrm{H}), 3.96(\mathrm{dd}, J=8.0,5.9 \mathrm{~Hz}, 1 \mathrm{H}), 3.74(\mathrm{~s}$, 3H), 3.42 (s, 3H), 2.97 (dd, $J=16.4,5.6 \mathrm{~Hz}, 1 \mathrm{H}), 2.74$ (dd, $J=16.4,8.1 \mathrm{~Hz}, 1 \mathrm{H}), 2.56$ (dd, 13.7, 8.4 Hz, 1H), $2.44(\mathrm{dd}, 13.7,7.9 \mathrm{~Hz}, 1 \mathrm{H}), 2.10(\mathrm{br}, 1 \mathrm{H}) ;{ }^{13} \mathrm{C} \mathrm{NMR}\left(75 \mathrm{MHz}, \mathrm{CDCl}_{3}\right) \delta$ $194.6,174.2$, 148.1, 143.2, 141.2, 133.6, 131.6, 131.2, 129.5, 124.5, 82.5, 59.9, 58.4, 52.4, 
52.2, 43.8, 40.9; HRMS (ESI): Calc. for $\mathrm{C}_{17} \mathrm{H}_{19} \mathrm{~N}_{2} \mathrm{O}_{6}(\mathrm{M}+\mathrm{H})^{+}$: 347,1238; found: 347, 1245 .

$[\alpha]_{\mathrm{D}}\left(25^{\circ} \mathrm{C}, \mathrm{c}=1.5 \mathrm{mg} \cdot \mathrm{mL}-1, \mathrm{CH}_{2} \mathrm{Cl}_{2}\right):-49^{\circ}$.

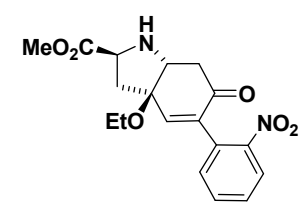

(2S,3aR,7aR)-methyl

3a-ethoxy-2,3,3a,6,7,7a-hexahydro-5-(2-

nitrophenyl)-6-oxo-1H-indole-2-carboxylate (12f): (45 mg, 63\% yield) $\mathrm{RMN}{ }^{1} \mathrm{H}\left(\mathrm{CDCl}_{3}\right.$, $300 \mathrm{MHz}) \delta(\mathrm{ppm}): 1.25(\mathrm{t}, 3 \mathrm{H}, J=6.9 \mathrm{~Hz}) ; 2.45(\mathrm{dd}, 1 \mathrm{H}, J=8.4,13.6 \mathrm{~Hz}), 2.58(\mathrm{dd}, 1 \mathrm{H}, J$ $=8.4,13.6 \mathrm{~Hz}), 2.75(\mathrm{dd}, 1 \mathrm{H}, J=7.7,16.4 \mathrm{~Hz}), 2.98(\mathrm{dd}, 1 \mathrm{H}, J=5.6,16.4 \mathrm{~Hz}), 3.65(\mathrm{~m}, 2 \mathrm{H})$, $3.76(\mathrm{~s}, 3 \mathrm{H}), 3.95(\mathrm{dd}, 1 \mathrm{H}, J=5.7,7.1 \mathrm{~Hz}), 4.18(\mathrm{t}, 1 \mathrm{H}, J=8.0 \mathrm{~Hz}), 6.76(\mathrm{~s}, 1 \mathrm{H}), 7.29$ (dd, $1 \mathrm{H}, J=8.1,1.3 \mathrm{~Hz}), 7.55(\mathrm{td}, 1 \mathrm{H}, J=8.1,1.3 \mathrm{~Hz}), 7.67$ (td, $1 \mathrm{H}, J=8.1,1.3 \mathrm{~Hz}), 8.10(\mathrm{~d}$, $1 \mathrm{H}, J=8.1 \mathrm{~Hz}) . \mathrm{RMN}{ }^{13} \mathrm{C}\left(\mathrm{CDCl}_{3}, 300 \mathrm{MHz}\right) \delta(\mathrm{ppm}): 16.1,41.3,43.6,52.5,58.5,60.2$, 60.6, 82.0, 124.6, 129.5, 131.2, 131.7, 133.7, 140.7, 143.9, 148.2, 174.1, 194.6; HRMS (ESI): Calc. for $\mathrm{C}_{18} \mathrm{H}_{21} \mathrm{~N}_{2} \mathrm{O}_{6}(\mathrm{M}+\mathrm{H})^{+}: 361,1394$; found: 361,1375 . [ $\left.\alpha\right]_{\mathrm{D}}\left(25^{\circ} \mathrm{C}, \mathrm{c}=2.7 \mathrm{mg} \cdot \mathrm{mL}-1\right.$, $\left.\mathrm{CH}_{2} \mathrm{Cl}_{2}\right):-51.9^{\circ}$.



2,3,4,4a,8,8a-hexahydro-4a-methoxy-6-(4-nitrophenyl)quinolin-7(1H)-

one (12g): (44 mg, 73\% yield) . ${ }^{1} \mathrm{H}$ NMR (300 MHz, $\left.\mathrm{CDCl}_{3}\right) \delta 8.21$ (d, J=8.9 Hz, 1H), 7.53 $(\mathrm{d}, J=8.9 \mathrm{~Hz}, 1 \mathrm{H}), 6.88(\mathrm{~s}, 1 \mathrm{H}), 3.44(\mathrm{dd}, J=7.6,4.0 \mathrm{~Hz}, 1 \mathrm{H}), 3.39$ (s, 1H), 3.06 (dd, $J=$ 16.6, 4.0 Hz, 1H), $2.98-2.73(\mathrm{~m}, 1 \mathrm{H}), 2.68(\mathrm{dd}, J=16.7,7.7 \mathrm{~Hz}, 1 \mathrm{H}), 2.16-2.01(\mathrm{~m}, 1 \mathrm{H})$, $1.92-1.70(\mathrm{~m}, 2 \mathrm{H}), 1.55-1.42(\mathrm{~m}, 1 \mathrm{H})$.

${ }^{13} \mathrm{C}$ NMR $\left(75 \mathrm{MHz}, \mathrm{CDCl}_{3}\right) \delta 195.9,150.1,147.3,141.7,139.5,129.5,123.0,72.5,56.1$, 50.1, 42.4, 41.2, 31.4, 21.9. HRMS (ESI): Calc. for $\mathrm{C}_{16} \mathrm{H}_{19} \mathrm{~N}_{2} \mathrm{O}_{4}(\mathrm{M}+\mathrm{H})^{+}$: 303.1339; found: 303.1345 .

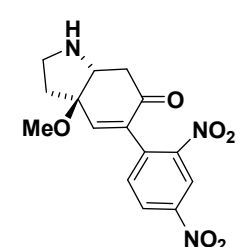

$\mathrm{NO}_{2}$ (3aR,7aR)-1,2,3,3a,7,7a-hexahydro-3a-methoxy-5-(2,4-dinitrophenyl)indol6-one (12h): (43 mg, 64\% yield). ${ }^{1} \mathrm{H}$ NMR (300 MHz, $\left.\mathrm{CDCl}_{3}\right) \delta 8.90(\mathrm{~d}, J=2.2 \mathrm{~Hz}, 1 \mathrm{H})$, 8.48 (dd, $J=8.3,2.4 \mathrm{~Hz}, 1 \mathrm{H}), 7.54$ (d, $J=8.3 \mathrm{~Hz}, 1 \mathrm{H}), 6.95$ (s, 1H), 3.91 (dd, $J=6.1,5.9 \mathrm{~Hz}$, 1H), $3.41(\mathrm{~s}, 3 \mathrm{H}), 3.23(\mathrm{~m}, 2 \mathrm{H}), 2.91(\mathrm{dd}, J=16.2,5.6 \mathrm{~Hz}, 1 \mathrm{H}), 2.67$ (dd, 16.2, 8.0 Hz, 1H), 
2.34-2.16 (m, 2H); ${ }^{13} \mathrm{C}$ NMR $\left(75 \mathrm{MHz}, \mathrm{CDCl}_{3}\right) \delta 194.2,148.4,147.8,146.6,138.7,137.0$, 132.9, 127.6, 119.9, 82.7, 60.0, 52.3, 44.5, 42.2, 38.0; HRMS (ESI): Calc. for $\mathrm{C}_{15} \mathrm{H}_{16} \mathrm{~N}_{3} \mathrm{O}_{6}$ $(\mathrm{M}+\mathrm{H})^{+}:$334.1034; found: 334.1042 .

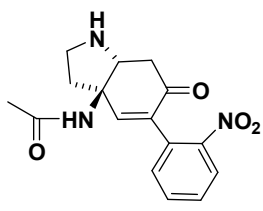

N-((3aR,7aR)-2,3,3a,6,7,7a-hexahydro-5-(2-nitrophenyl)-6-oxo-1H-indol3a-yl)acetamide (12i): (47.5 mg, 76\% yield). $\mathrm{RMN}{ }^{1} \mathrm{H}\left(\mathrm{CDCl}_{3}, 300 \mathrm{MHz}\right) \delta(\mathrm{ppm}): 2.05$ (s, $3 \mathrm{H}) ; 2.42(\mathrm{t}, 2 \mathrm{H}, \mathrm{J}=7.6 \mathrm{~Hz}), 2.71(\mathrm{dd}, 1 \mathrm{H}, \mathrm{J}=6.75,16.4 \mathrm{~Hz}), 2.95(\mathrm{dd}, 1 \mathrm{H}, \mathrm{J}=5.10,16.1 \mathrm{~Hz})$, 3.25 (m, 2H), 3.87 (t, 1H, J=6.3 Hz), 6.27 (br, 1H), 7.04(s, 1H), 7.34 (d, 1H, J=8.1 Hz), 7.50 $(\mathrm{t}, 1 \mathrm{H}, \mathrm{J}=8.1 \mathrm{~Hz}), 7.62(\mathrm{t}, 1 \mathrm{H}, \mathrm{J}=8.1 \mathrm{~Hz}), 8.00(\mathrm{~d}, 1 \mathrm{H}, \mathrm{J}=8.1 \mathrm{~Hz}) ; \mathrm{RMN}{ }^{13} \mathrm{C}\left(\mathrm{CDCl}_{3}, 300\right.$ $\mathrm{MHz}) \delta(\mathrm{ppm}): 23.8,38.2,41.3,44.2,61.1,62.6,124.3,129.2,131.3,132.0,133.7,137.40$, 144.9, 148.2, 170.4, 194.8. HRMS (ESI): Calc. for $\mathrm{C}_{16} \mathrm{H}_{18} \mathrm{~N}_{3} \mathrm{O}_{4}(\mathrm{M}+\mathrm{H})^{+}:$316.1292; found: 316.1303 .

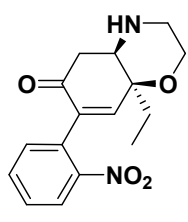

8a-ethyl-3,4,4a,5-tetrahydro-5-(2-nitrophenyl)-2H-benzo $[b][1,4]$ oxazin6(8aH)-one (12j): (45 mg, 75\% yield). $\mathrm{RMN}{ }^{1} \mathrm{H}\left(\mathrm{CDCl}_{3}, 300 \mathrm{MHz}\right) \delta(\mathrm{ppm}): 1.11(\mathrm{t}, 3 \mathrm{H}, \mathrm{J}=$ $7.5 \mathrm{~Hz}) ; 1.89(\mathrm{~m}, 1 \mathrm{H}), 1.98(\mathrm{~m}, 1 \mathrm{H}), 2.56(\mathrm{dd}, 1 \mathrm{H}, \mathrm{J}=2.4,17.4 \mathrm{~Hz}), 3.02(\mathrm{~m}, 3 \mathrm{H}), 3.46$ (m, 1H), 3.82 (d, 2H, J=5.9 Hz), 6.77 (d, J=2.4 Hz, 1H), 7.41 (d, 1H, J=8.1 Hz), $7.54(\mathrm{~m}, 1 \mathrm{H})$, 7.67(t, $1 \mathrm{H}, \mathrm{J}=8.1 \mathrm{~Hz}), 8.09(\mathrm{~d}, 1 \mathrm{H}, \mathrm{J}=8.1 \mathrm{~Hz}) . \mathrm{RMN}{ }^{13} \mathrm{C}\left(\mathrm{CDCl}_{3}, 300 \mathrm{MHz}\right) \delta(\mathrm{ppm}): 7.6$, $31.9,42.5,45.1,57.0,63.9,74.8,124.4,129.2,131.3,131.8,133.6,140.3,148.4,149.2$, 193.5. HRMS (ESI): Calc. for $\mathrm{C}_{16} \mathrm{H}_{19} \mathrm{~N}_{2} \mathrm{O}_{4}(\mathrm{M}+\mathrm{H})^{+}$: 303.1339 ; found: 303.1326 .

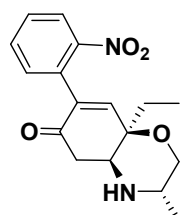

8a-ethyl-3,4,4a,5-tetrahydro-3-methyl-7-(2-nitrophenyl)-2H-benzo[b][1,4]oxazin-6(8aH)-one (12k): (46 mg, 73\% yield). $\mathrm{RMN}{ }^{1} \mathrm{H}\left(\mathrm{CDCl}_{3}, 300 \mathrm{MHz}\right) \delta(\mathrm{ppm}): 1.01$ (d, $3 \mathrm{H}, \mathrm{J}=6.4 \mathrm{~Hz}), 1.12$ (t, 3H, J= $7.4 \mathrm{~Hz}), 1.87(\mathrm{~m}, 1 \mathrm{H}), 1.88(\mathrm{~m}, 1 \mathrm{H}), 2.52$ (dd, 1H, J=2.4, 17.4 Hz), 2.93 (dd, 1H, J=4.0, 17.4 Hz), $3.03(\mathrm{~m}, 1 \mathrm{H}), 3.32$ (t, 1H, J=10.6 Hz), 3.48 (q, 1H, J=2.6 Hz), 3.75 (dd, 1H, J=3.0, 11.2 Hz), 6.76 (d, 1H, J=2.6 Hz), 7.33 (dd, 1H, J= 7.2, 1.3 Hz), 7.54 
$(\mathrm{td}, 1 \mathrm{H}, \mathrm{J}=7.2,1.3 \mathrm{~Hz}), 7.67(\mathrm{td}, 1 \mathrm{H}, \mathrm{J}=7.2,1.3 \mathrm{~Hz}), 8.09(\mathrm{dd}, 1 \mathrm{H}, \mathrm{J}=7.2,1.3 \mathrm{~Hz})$; RMN ${ }^{13} \mathrm{C}$ $\left(\mathrm{CDCl}_{3}, 300 \mathrm{MHz}\right) \delta(\mathrm{ppm}):$ 7.7, 17.2, 31.7, 42.9, 49.7, 57.2, 70.2, 74.2, 124.4, 129.2, 131.4, 131.7, 133.5, 140.1, 148.5, 149.9, 194.0; HRMS (ESI): Calc. for $\mathrm{C}_{17} \mathrm{H}_{21} \mathrm{~N}_{2} \mathrm{O}_{4}(\mathrm{M}+\mathrm{H})^{+}$: 317.1496; found: 317.1485 . $[\alpha]_{\mathrm{D}}\left(25^{\circ} \mathrm{C}, \mathrm{c}=1.9 \mathrm{mg} \cdot \mathrm{mL}-1, \mathrm{CH}_{2} \mathrm{Cl}_{2}\right)$ : $+17.9^{\circ}$.

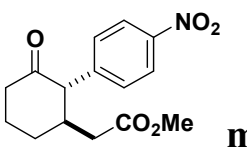

methyl 2-(4-nitrophenyl)-3-oxocyclohexyl)acetate (15): (77 $\mathrm{mg}, 53 \%$ yield). ${ }^{1} \mathrm{H}\left(\mathrm{CDCl}_{3}, 300 \mathrm{MHz}\right) \delta(\mathrm{ppm}): 8.21(\mathrm{~d}, J=8.1 \mathrm{~Hz}, 1 \mathrm{H}) ; 7.27(\mathrm{~d}, J=8.1 \mathrm{~Hz}, 1 \mathrm{H}), 3.66(\mathrm{~d}, J$ $=12.2 \mathrm{~Hz}, 1 \mathrm{H}), 3.58(\mathrm{~s}, 3 \mathrm{H}), 2.53(\mathrm{~m}, 3 \mathrm{H}), 2.13(\mathrm{~m}, 3 \mathrm{H}), 1.78(\mathrm{~m}, 2 \mathrm{H}) . \mathrm{RMN}{ }^{13} \mathrm{C}\left(\mathrm{CDCl}_{3}\right.$, $300 \mathrm{MHz}) \delta(\mathrm{ppm}): 207.5,171.8,147.1,144.1,130.4,123.5,62.0,51.5,41.9,41.5,38.7,31.0$, 25.3; HRMS (ESI): Calc. for $\mathrm{C}_{15} \mathrm{H}_{18} \mathrm{NO}_{5}(\mathrm{M}+\mathrm{H})^{+}: 292.1179$; found: 292.1184 .

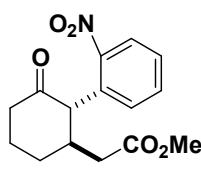

methyl 2-(2-(2-nitrophenyl)-3-oxocyclohexyl)acetate (17): (72 $\mathrm{mg}, 50 \%$ yield). ${ }^{1} \mathrm{H}\left(\mathrm{CDCl}_{3}, 300 \mathrm{MHz}\right) \delta(\mathrm{ppm}): 7.97(\mathrm{~d}, J=8.1 \mathrm{~Hz}, 1 \mathrm{H}) ; 7.61(\mathrm{t}, J=8.1 \mathrm{~Hz}, 1 \mathrm{H}), 7.44$ (t, $J=8.1 \mathrm{~Hz}, 1 \mathrm{H}), 7.32(\mathrm{~d}, J=8.1 \mathrm{~Hz}, 1 \mathrm{H}), 4.05(\mathrm{~d}, J=11.4 \mathrm{~Hz}, 1 \mathrm{H}), 2.71(\mathrm{~m}, 1 \mathrm{H}), 2.61(\mathrm{~d}$, $J=14.9 \mathrm{~Hz}, 1 \mathrm{H}), 2.47(\mathrm{dt}, J=13.6,5.6 \mathrm{~Hz}, 1 \mathrm{H}), 2.22(\mathrm{~d}, J=6.0 \mathrm{~Hz}, 1 \mathrm{H}), 2.16(\mathrm{~d}, J=11.7$ $\mathrm{Hz}, 1 \mathrm{H}), 1.91$ (q, $J=11.3 \mathrm{~Hz}, 1 \mathrm{H}), 1.65$ (q, $J=11.3 \mathrm{~Hz}, 1 \mathrm{H}) . \mathrm{RMN}^{13} \mathrm{C}\left(\mathrm{CDCl}_{3}, 300 \mathrm{MHz}\right)$ $\delta(\mathrm{ppm}): 206.6,172.0,149.9,132.8,131.9,128.1,125.0,58.2,51.5,41.1,40.5,39.0,31.1$, 24.4; HRMS (ESI): Calc. for $\mathrm{C}_{15} \mathrm{H}_{18} \mathrm{NO}_{5}(\mathrm{M}+\mathrm{H})^{+}: 292.1179$; found: 292.1184 .



4-benzyl-3-(2-(2-(4-nitrophenyl)-3-oxocyclohexyl)acetyl)oxazolidin-2-one (20): (on $0.1 \mathrm{mmol}, 18 \mathrm{mg}, 42 \%$ yield) ${ }^{1} \mathrm{H}\left(\mathrm{CDCl}_{3}, 300 \mathrm{MHz}\right) \delta(\mathrm{ppm}): 8.22(\mathrm{~d}, J=8.1 \mathrm{~Hz}$, 2H); $7.33(2, J=8.1 \mathrm{~Hz}, 2 \mathrm{H}), 7.27(\mathrm{c}, 3 \mathrm{H}), 7.12(\mathrm{~d}, J=8.1 \mathrm{~Hz}, 2 \mathrm{H}), 4.58(\mathrm{~m}, 1 \mathrm{H}), 4.13$ (m, 2H), $3.68(\mathrm{~d}, J=11.7 \mathrm{~Hz}, 1 \mathrm{H}), 2.16(\mathrm{dd}, J=13.6,2.6 \mathrm{~Hz}, 1 \mathrm{H}), 2.94-2.42$ (m, 6H), 2.19 (m, $2 \mathrm{H}), 1.89$ (q, $J=11.3 \mathrm{~Hz}, 1 \mathrm{H}), 1.71$ (q, $J=11.3 \mathrm{~Hz}, 1 \mathrm{H}) . \mathrm{RMN}{ }^{13} \mathrm{C}\left(\mathrm{CDCl}_{3}, 300 \mathrm{MHz}\right)$ $\delta(\mathrm{ppm}): 207.5,171.0,153.2,147.2,144.1,134.9,130.6,129.1,128.9,127.4,123.5,66.2$, 62.3, 55.1, 41.6, 41.0, 39.9, 37.8, 31.3, 25.4; HRMS (ESI): Calc. for $\mathrm{C}_{24} \mathrm{H}_{25} \mathrm{~N}_{2} \mathrm{O}_{6}(\mathrm{M}+\mathrm{H})^{+}$: 437.1707; found: 437.1719. $[\alpha]_{\mathrm{D}}\left(25^{\circ} \mathrm{C}, \mathrm{c}=1.5 \mathrm{mg} \cdot \mathrm{mL}-1, \mathrm{CH}_{2} \mathrm{Cl}_{2}\right):+16.7^{\circ}$. 


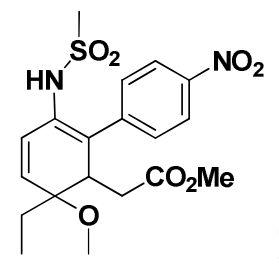

\section{Methyl 2-(3-ethyl-6-(methylsulfonamido)-4'-nitro-2,3-dihydro-[1,1'-}

biphenyl]-2-yl)acetate (23a): Pale yellow oil: $0.038 \mathrm{mmol}, 16.2 \mathrm{mg}, 57 \%$ yield; ${ }^{1} \mathrm{H}$ NMR $\left(300 \mathrm{MHz}, \mathrm{CDCl}_{3}\right) \delta 8.30(\mathrm{~d}, J=8.7 \mathrm{~Hz}, 2 \mathrm{H}), 7.44(\mathrm{~d}, J=8.7 \mathrm{~Hz}, 2 \mathrm{H}), 6.33(\mathrm{~d}, J=10.2 \mathrm{~Hz}$, $1 \mathrm{H}), 6.04-5.96(\mathrm{~m}, 2 \mathrm{H}), 3.36(\mathrm{~s}, 3 \mathrm{H}), 3.27(\mathrm{~s}, 3 \mathrm{H}$ and $1 \mathrm{H}), 2.86(\mathrm{~s}, 3 \mathrm{H}), 2.80(\mathrm{dd}, J=15.5$, $5.1 \mathrm{~Hz}, 1 \mathrm{H}), 2.29(\mathrm{dd}, J=15.4,8.0 \mathrm{~Hz}, 1 \mathrm{H}), 2.01-1.83(\mathrm{~m}, 2 \mathrm{H}), 1.01(\mathrm{t}, J=7.4 \mathrm{~Hz}, 3 \mathrm{H}) .{ }^{13} \mathrm{C}$ NMR (75 MHz, $\left.\mathrm{CDCl}_{3}\right) \delta 172.9,147.3,143.6,135.1,129.7,128.1,126.2,124.3,121.4,78.2$, 51.4, 49.6, 43.4, 41.8, 31.9, 25.7, 7.7. HRMS (ESI): Calc. for $\mathrm{C}_{18} \mathrm{H}_{21} \mathrm{~N}_{2} \mathrm{O}_{6} \mathrm{~S}(\mathrm{M}+\mathrm{H}-\mathrm{MeOH})^{+}$: 425.13771; found: 425.1380 .

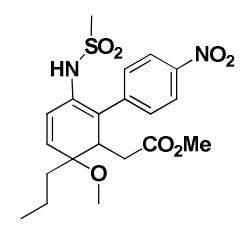

Methyl 2-(6-(methylsulfonamido)-4'-nitro-3-propyl-2,3-dihydro-[1,1'-

biphenyl]-2-yl)acetate (23b): Pale yellow oil: $0.031 \mathrm{mmol}, 14 \mathrm{mg}, 52 \%$ yield; ${ }^{1} \mathrm{H}$ NMR (300 $\left.\mathrm{MHz} \mathrm{CDCl}_{3}\right) \delta 8.30(\mathrm{~d}, J=8.6 \mathrm{~Hz}, 2 \mathrm{H}), 7.44(\mathrm{~d}, J=8.6 \mathrm{~Hz}, 2 \mathrm{H}), 6.32(\mathrm{~d}, J=10.2 \mathrm{~Hz}, 1 \mathrm{H})$, $5.98(\mathrm{~d}, J=10.9 \mathrm{~Hz}, 2 \mathrm{H}), 3.35(\mathrm{~s}, 3 \mathrm{H}), 3.25(\mathrm{~s}, 3 \mathrm{H}$ and $1 \mathrm{H}), 2.87(\mathrm{~s}, 3 \mathrm{H}), 2.79(\mathrm{dd}, J=15.4$, $4.9 \mathrm{~Hz}, 1 \mathrm{H}), 2.29$ (dd, $J=15.4,8.1 \mathrm{~Hz}, 1 \mathrm{H}), 1.90-1.80(\mathrm{~m}, 2 \mathrm{H}), 1.58-1.35$ (m, 2H), 0.99 (t, $J$ $=7.3 \mathrm{~Hz}, 3 \mathrm{H}) .{ }^{13} \mathrm{C} \mathrm{NMR}\left(75 \mathrm{MHz}, \mathrm{CDCl}_{3}\right) \delta 172.9,147.3,143.6,135.4,129.7,128.1,126.1$, 124.3, 121.2, 78.1, 51.4, 49.8, 43.7, 41.8, 35.5, 31.8, 16.8, 14.7. HRMS (ESI): Calc. for $\mathrm{C}_{19} \mathrm{H}_{23} \mathrm{~N}_{2} \mathrm{O}_{6} \mathrm{~S}(\mathrm{M}+\mathrm{H}-\mathrm{MeOH})^{+}: 407.1271$; found: 407.1264 .

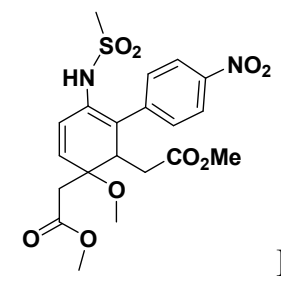

\section{Dimethyl 2,2'-(3-methoxy-6-(methylsulfonamido)-4'-nitro-1,2,3,6-}

tetrahydro-[1,1'-biphenyl]-2,3-diyl)diacetate (23c): Pale yellow oil: $0.026 \mathrm{mmol}, 12.49 \mathrm{mg}$, 49\% yield; ${ }^{1} \mathrm{H}$ NMR $\left(300 \mathrm{MHz}, \mathrm{CDCl}_{3}\right) \delta 8.31(\mathrm{~d}, J=8.8 \mathrm{~Hz}, 2 \mathrm{H}), 7.64(\mathrm{~d}, J=8.8 \mathrm{~Hz}, 2 \mathrm{H})$, $6.38(\mathrm{~d}, J=10.2 \mathrm{~Hz}, 1 \mathrm{H}), 6.22-6.06(\mathrm{~m}, 2 \mathrm{H}), 3.77(\mathrm{~s}, 3 \mathrm{H}), 3.63(\mathrm{dd}, J=11.8,4.3 \mathrm{~Hz}, 1 \mathrm{H})$, $3.40(\mathrm{~s}, 3 \mathrm{H}), 3.34(\mathrm{~s}, 3 \mathrm{H}), 3.05-2.87(\mathrm{~m}, 2 \mathrm{H}), 2.85(\mathrm{~s}, 3 \mathrm{H}), 2.82-2.73(\mathrm{~m}, 1 \mathrm{H}), 2.25(\mathrm{dd}, J=$ 15.1, 7.5 Hz, 1H). ${ }^{13} \mathrm{C} \mathrm{NMR}\left(75 \mathrm{MHz}, \mathrm{CDCl}_{3}\right) \delta 172.5,170.1,147.4,143.2,131.5,130.2$, 
128.2, 127.1, 124.3, 122.5, 77.5, 52.1, 51.4, 50.5, 43.7, 41.8, 37.9, 31.4. . HRMS (ESI): Calc. for $\mathrm{C}_{20} \mathrm{H}_{24} \mathrm{~N}_{2} \mathrm{NaO}_{9} \mathrm{~S}(\mathrm{M}+\mathrm{Na})^{+}$: 491.1095; found: 491.1096 .

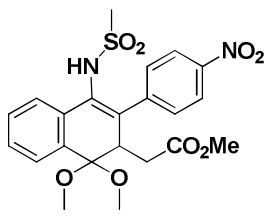

Methyl 2-(1,1-dimethoxy-4-(methylsulfonamido)-3-(4-nitrophenyl)-1,2dihydronaphthalen-2-yl)acetate (23d): Pale yellow oil: $0.030 \mathrm{mmol}, 14.65 \mathrm{mg}, 79 \%$ yield; ${ }^{1} \mathrm{H}$ NMR $\left(300 \mathrm{MHz}, \mathrm{CDCl}_{3}\right) \delta 8.32(\mathrm{~d}, J=8.7 \mathrm{~Hz}, 2 \mathrm{H}), 7.74(\mathrm{dd}, J=6.0,1.7 \mathrm{~Hz}, 2 \mathrm{H})$, $7.59(\mathrm{~d}, J=8.7 \mathrm{~Hz}, 2 \mathrm{H}), 7.46-7.41(\mathrm{~m}, 2 \mathrm{H}), 6.44(\mathrm{~s}, 1 \mathrm{H}), 3.57(\mathrm{dd}, J=9.8,4.1 \mathrm{~Hz}, 1 \mathrm{H}), 3.47$ (s, 3H), 3.28 (s, 3H), 2.99 (s, 3H), 2.52 (dd, $J=15.1,3.9 \mathrm{~Hz}, 1 \mathrm{H}), 2.47$ (s, 3H), $2.10-1.98$ $(\mathrm{m}, 1 \mathrm{H}) .{ }^{13} \mathrm{C} \mathrm{NMR}\left(75 \mathrm{MHz}, \mathrm{CDCl}_{3}\right) \delta 171.8,147.2,145.2,136.4,132.8,131.5,129.7,128.9$, 128.8, 128.7, 99.7, 51.5, 50.2, 48.4, 44.0, 40.2, 33.6. HRMS (ESI): Calc. for $\mathrm{C}_{21} \mathrm{H}_{20} \mathrm{~N}_{2} \mathrm{NaO}_{7} \mathrm{~S}(\mathrm{M}+\mathrm{Na}-\mathrm{MeOH})^{+}$: 467.0876; found: 467.0883 .



Methyl 2-(4'-(methylsulfonamido)-3'-(4-nitrophenyl)-2'Hspiro[[1,3]dioxolane-2,1'-naphthalen]-2-yl)acetate (23e): Pale yellow oil: 0.044 mmol, 21 mg, 62\% yield; ${ }^{1} \mathrm{H}$ NMR (300 MHz, $\left.\mathrm{CDCl}_{3}\right) \delta 8.32(\mathrm{~d}, J=8.7 \mathrm{~Hz}, 2 \mathrm{H}), 7.73-7.67(\mathrm{~m}, 1 \mathrm{H})$, $7.59(\mathrm{dd}, J=8.8,1.9 \mathrm{~Hz}, 3 \mathrm{H}), 7.51-7.39(\mathrm{~m}, 2 \mathrm{H}), 6.28(\mathrm{~s}, 1 \mathrm{H}), 4.34-4.26(\mathrm{~m}, 1 \mathrm{H}), 4.22-$ $4.10(\mathrm{~m}, 2 \mathrm{H}), 4.07-3.98(\mathrm{~m}, 1 \mathrm{H}), 3.50-3.47(\mathrm{~m}, 1 \mathrm{H}), 3.46(\mathrm{~s}, 3 \mathrm{H}$ and $1 \mathrm{H}), 2.63-2.57(\mathrm{~m}$, $1 \mathrm{H}), 2.54(\mathrm{~s}, 3 \mathrm{H}), 2.19(\mathrm{dd}, J=15.5,5.9 \mathrm{~Hz}, 1 \mathrm{H}) .{ }^{13} \mathrm{C} \mathrm{NMR}\left(75 \mathrm{MHz}, \mathrm{CDCl}_{3}\right) \delta 171.9,147.3$, $144.9,136.1,134.3,131.7,129.9,129.5,129.3,129.1,125.4,124.8,124.1,107.7,66.4,64.6$, 51.7, 46.3, 40.7, 33.2. HRMS (ESI): Calc. for $\mathrm{C}_{22} \mathrm{H}_{22} \mathrm{~N}_{2} \mathrm{NaO}_{8} \mathrm{~S}(\mathrm{M}+\mathrm{Na})^{+}: 497.0989$ found: 497.0993.

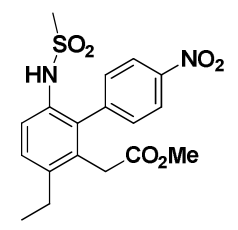

\section{Methyl 2-(-3-ethyl-6-(methylsulfonamido)-4'-nitro-[1,1'-biphenyl]-2-}

yl)acetate (25a): Pale yellow oil: $0.027 \mathrm{mmol}, 10.7 \mathrm{mg}, 83 \%$ yield; ${ }^{1} \mathrm{H}$ NMR (300 MHz, $\left.\mathrm{CDCl}_{3}\right) \delta 8.38(\mathrm{~d}, J=8.7 \mathrm{~Hz}, 2 \mathrm{H}), 7.58(\mathrm{~d}, J=8.5 \mathrm{~Hz}, 1 \mathrm{H}), 7.46(\mathrm{~d}, J=8.7 \mathrm{~Hz}, 2 \mathrm{H}), 7.36(\mathrm{~d}$, $J=8.5 \mathrm{~Hz}, 1 \mathrm{H}), 5.72(\mathrm{~s}, 1 \mathrm{H}), 3.62(\mathrm{~s}, 3 \mathrm{H}), 3.38(\mathrm{~s}, 2 \mathrm{H}), 2.94(\mathrm{~s}, 3 \mathrm{H}), 2.65(\mathrm{q}, J=7.5 \mathrm{~Hz}, 2 \mathrm{H})$, 
$1.26(\mathrm{t}, J=7.5 \mathrm{~Hz}, 4 \mathrm{H}) .{ }^{13} \mathrm{C} \mathrm{NMR}\left(75 \mathrm{MHz}, \mathrm{CDCl}_{3}\right) \delta 171.3,148.1,140.5,132.5,132.1$, 131.5, 131.1, 124.6, 120.1, 52.2, 40.3, 35.7, 25.8, 14.6. HRMS (ESI): Calc. for $\mathrm{C}_{36} \mathrm{H}_{40} \mathrm{~N}_{4} \mathrm{NaO}_{12} \mathrm{~S}_{2}(2 \mathrm{M}+\mathrm{Na})^{+}: 807.1976$ found: 807.1975 .

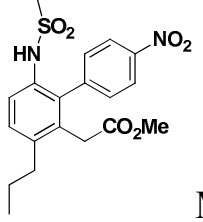

Methyl 2-(-6-(methylsulfonamido)-4'-nitro-3-propyl-[1,1'-biphenyl]-2yl)acetate (25b): Pale yellow oil: $0.027 \mathrm{mmol}, 11 \mathrm{mg}, 85 \%$ yield; ${ }^{1} \mathrm{H}$ NMR (300 MHz, $\left.\mathrm{CDCl}_{3}\right) \delta 8.38(\mathrm{~d}, J=8.6 \mathrm{~Hz}, 2 \mathrm{H}), 7.56(\mathrm{~d}, J=8.5 \mathrm{~Hz}, 1 \mathrm{H}), 7.46(\mathrm{~d}, J=8.6 \mathrm{~Hz}, 2 \mathrm{H}), 7.33(\mathrm{~d}$, $J=8.5 \mathrm{~Hz}, 1 \mathrm{H}), 5.71(\mathrm{~s}, 1 \mathrm{H}), 3.61(\mathrm{~s}, 3 \mathrm{H}), 3.38(\mathrm{~s}, 2 \mathrm{H}), 2.94(\mathrm{~s}, 3 \mathrm{H}), 2.63-2.54(\mathrm{~m}, 2 \mathrm{H})$, $1.63(\mathrm{dq}, J=14.9,7.4 \mathrm{~Hz}, 2 \mathrm{H}), 1.02(\mathrm{t}, J=7.3 \mathrm{~Hz}, 3 \mathrm{H}) .{ }^{13} \mathrm{C} \mathrm{NMR}\left(75 \mathrm{MHz}, \mathrm{CDCl}_{3}\right) \delta 171.3$, 148.1, 143.4, 139.1, 132.4, 132.1, 131.6, 131.1, 130.6, 124.5, 119.8, 52.1, 40.3, 35.8, 35.1, 23.7, 14.1. HRMS (ESI): Calc. for $\mathrm{C}_{19} \mathrm{H}_{22} \mathrm{~N}_{2} \mathrm{NaO}_{6} \mathrm{~S}(\mathrm{M}+\mathrm{Na})^{+}$: 429.1091 found: 429.1091.

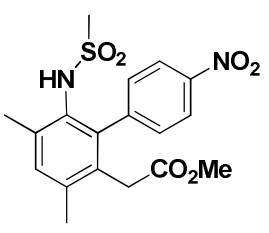

Methyl 2-(3,5-dimethyl-6-(methylsulfonamido)-4'-nitro-[1,1'-biphenyl]-

2-yl)acetate (25c): Pale yellow oil: $0.042 \mathrm{mmol}, 16.65 \mathrm{mg}, 60 \%$ yield; ${ }^{1} \mathrm{H}$ NMR $(300 \mathrm{MHz}$, $\left.\mathrm{CDCl}_{3}\right) \delta 8.40(\mathrm{~d}, J=8.7 \mathrm{~Hz}, 2 \mathrm{H}), 7.54(\mathrm{~d}, J=8.7 \mathrm{~Hz}, 2 \mathrm{H}), 7.24(\mathrm{~s}, 1 \mathrm{H}), 5.69(\mathrm{~s}, 1 \mathrm{H}), 3.61(\mathrm{~s}$, $3 \mathrm{H}), 3.36(\mathrm{~s}, 2 \mathrm{H}), 2.47(\mathrm{~s}, 3 \mathrm{H}), 2.46(\mathrm{~s}, 3 \mathrm{H}), 2.32(\mathrm{~s}, 3 \mathrm{H}) .{ }^{13} \mathrm{C} \mathrm{NMR}\left(75 \mathrm{MHz}, \mathrm{CDCl}_{3}\right) \delta$ 171.2, 147.4, 145.7, 139.9, 137.8, 136.7, 133.3, 131.5, 130.3, 129.9, 52.1, 42.3, 36.3, 19.9, 19.3. HRMS (ESI): Calc. for $\mathrm{C}_{36} \mathrm{H}_{40} \mathrm{~N}_{4} \mathrm{NaO}_{12} \mathrm{~S}_{2}(2 \mathrm{M}+\mathrm{Na})^{+}: 807.1976$ found: 807.1967.

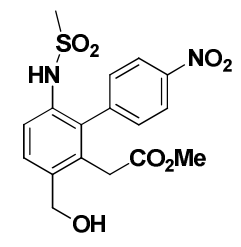

\section{Methyl 2-(3-(hydroxymethyl)-6-(methylsulfonamido)-4'-nitro-[1,1'-}

biphenyl]-2-yl)acetate (25d): Pale yellow oil: $0.040 \mathrm{mmol}, 15.9 \mathrm{mg}$, 55\% yield; ${ }^{1} \mathrm{H}$ NMR $\left(300 \mathrm{MHz}, \mathrm{CDCl}_{3}\right) \delta 8.36(\mathrm{~d}, J=8.7 \mathrm{~Hz}, 2 \mathrm{H}), 7.56(\mathrm{~d}, J=8.9 \mathrm{~Hz}, 1 \mathrm{H}), 7.46(\mathrm{~d}, J=8.7 \mathrm{~Hz}$, 2H), $7.00(\mathrm{~d}, J=9.0 \mathrm{~Hz}, 1 \mathrm{H}), 5.76(\mathrm{~s}, 1 \mathrm{H}), 3.89(\mathrm{~s}, 3 \mathrm{H}), 3.63(\mathrm{~s}, 3 \mathrm{H}$ and $1 \mathrm{H}), 3.34(\mathrm{~s}, 2 \mathrm{H})$, $2.83(\mathrm{~s}, 3 \mathrm{H}) .{ }^{13} \mathrm{C}$ NMR $\left(75 \mathrm{MHz}, \mathrm{CDCl}_{3}\right) \delta 171.6,156.1,147.9,143.1,135.7,130.9,126.4$, 
124.3, 124.0, 122.7, 111.2, 56.1, 52.1, 40.2, 33.3. HRMS (ESI): Calc. for $\mathrm{C}_{17} \mathrm{H}_{19} \mathrm{~N}_{2} \mathrm{O}_{7} \mathrm{~S}(\mathrm{M}+\mathrm{H})^{+}: 395.0907$ found: 395.0910 .

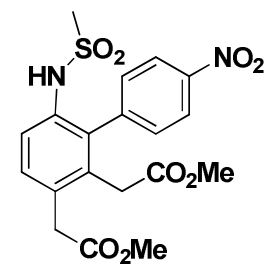

\section{Dimethyl 2,2'-(6-(methylsulfonamido)-4'-nitro-3-[1,1'-biphenyl]-2,3-}

diyl)diacetate (25e): Pale yellow oil: $0.022 \mathrm{mmol}, 9.8 \mathrm{mg}, 88 \%$ yield ${ }^{1} \mathrm{H}$ NMR $(300 \mathrm{MHz}$, $\left.\mathrm{CDCl}_{3}\right) \delta 8.39(\mathrm{~d}, J=8.7 \mathrm{~Hz}, 2 \mathrm{H}), 7.61(\mathrm{~d}, J=8.5 \mathrm{~Hz}, 1 \mathrm{H}), 7.48(\mathrm{~d}, J=8.7 \mathrm{~Hz}, 2 \mathrm{H}), 7.40(\mathrm{~d}$, $J=8.5 \mathrm{~Hz}, 1 \mathrm{H}), 5.78(\mathrm{~s}, 2 \mathrm{H}), 3.72(\mathrm{~s}, 3 \mathrm{H}), 3.69(\mathrm{~s}, 2 \mathrm{H}), 3.61(\mathrm{~s}, 3 \mathrm{H}), 3.43(\mathrm{~s}, 2 \mathrm{H}), 2.97$ (s, $3 \mathrm{H}) .{ }^{13} \mathrm{C} \mathrm{NMR}\left(75 \mathrm{MHz}, \mathrm{CDCl}_{3}\right) \delta 171.2,170.8,148.2,142.7,133.8,132.8,132.3,132.1$, 131.1, 130.8, 124.7, 119.2, 52.3, 52.2, 40.4, 38.7, 36.2. HRMS (ESI): Calc. for $\mathrm{C}_{19} \mathrm{H}_{20} \mathrm{~N}_{2} \mathrm{NaO}_{8} \mathrm{~S}(\mathrm{M}+\mathrm{Na})^{+}: 459.0833$ found: 459.0830 .

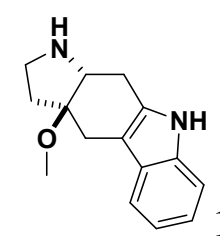

1,2,3,3a,4,9,10,10a-octahydro-3a-methoxypyrrolo[2,3-b]carbazole (30): (15 $\mathrm{mg}, 80 \%$ yield). ${ }^{1} \mathrm{H}\left(\mathrm{CDCl}_{3}, 300 \mathrm{MHz}\right) \delta(\mathrm{ppm}): 9.05$ (br, $\left.1 \mathrm{H}\right) ; 7.43(\mathrm{~d}, J=7.5 \mathrm{~Hz}, 1 \mathrm{H}), 7.34$ (d, $J=7.5 \mathrm{~Hz}, 1 \mathrm{H}), 7.17$ (t, 1H, $J=7.5 \mathrm{~Hz}), 7.10$ (t, 1H, $J=7.5 \mathrm{~Hz}), 3.90$ (br, 1H), 3.38 (br, 2H), 3.33 (s, 3H), 3.15 (d, J=15.8 Hz, 1H), 3.04 (d, 1H, J=15.8 Hz), 2.21 (br, 1H), 2.02 (br, 2H), 1.86 (br, $1 \mathrm{H}) . \mathrm{RMN}{ }^{13} \mathrm{C}\left(\mathrm{CDCl}_{3}, 300 \mathrm{MHz}\right) \delta(\mathrm{ppm}): 137.0,128.1,126.7,121.7,119.2$, 117.4, 111.1, 105.4, 83.6, 59.9, 49.7, 41.4, 30.1, 29.6, 24.7; HRMS (ESI): Calc. for $\mathrm{C}_{15} \mathrm{H}_{19} \mathrm{~N}_{2} \mathrm{O}(\mathrm{M}+\mathrm{H})^{+}:$243.1492; found: 243.1499 .

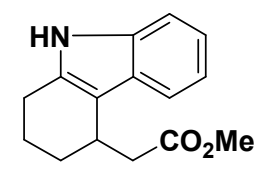

methyl 2-(6,7,8,9-tetrahydro-5H-carbazol-5-yl)acetate (31): (13 mg, 72\% yield). ${ }^{1} \mathrm{H}\left(\mathrm{CDCl}_{3}, 300 \mathrm{MHz}\right) \delta(\mathrm{ppm}): 7.76(\mathrm{br}, 1 \mathrm{H}) ; 7.51(\mathrm{~d}, J=7.4 \mathrm{~Hz}, 1 \mathrm{H}), 7.28(\mathrm{~d}, J=$ $7.4 \mathrm{~Hz}, 1 \mathrm{H}), 7.12$ (t, $J=6.7 \mathrm{~Hz}, 1 \mathrm{H}), 7.07$ (t, $J=6.7 \mathrm{~Hz}, 1 \mathrm{H}), 3.73(\mathrm{~s}, 3 \mathrm{H}), 3.53(\mathrm{~m}, 1 \mathrm{H}), 3.00$ (dd, $J=15.1,3.7 \mathrm{~Hz}, 1 \mathrm{H}), 2.71(\mathrm{~m}, 2 \mathrm{H}), 2.43(\mathrm{dd}, J=15.2,10.7 \mathrm{~Hz}, 1 \mathrm{H}), 2.00-1.72(\mathrm{~m}, 4 \mathrm{H})$. $\mathrm{RMN}{ }^{13} \mathrm{C}\left(\mathrm{CDCl}_{3}, 300 \mathrm{MHz}\right) \delta(\mathrm{ppm}): 173.6,135.7,134.5,126.7,121.0,119.2,118.0,112.1$, 
110.5, 51.5, 39.6, 28.9, 28.4, 23.2, 19.3; HRMS (ESI): Calc. for $\mathrm{C}_{15} \mathrm{H}_{21} \mathrm{~N}_{2} \mathrm{O}_{2}\left(\mathrm{M}+\mathrm{NH}_{4}\right)^{+}$: 261.1598; found: 261.1579 .

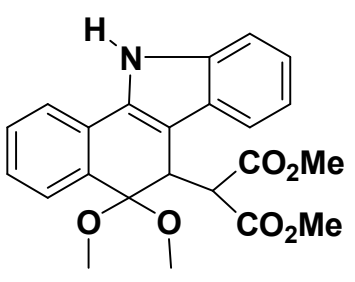

Dimethyl 2-(5,5-dimethoxy-6,11-dihydro-5H-benzo[a]carbazol-6-

yl)malonate (33): Pale yellow oil: $0.051 \mathrm{mmol}, 22 \mathrm{mg}, 82 \%$ yield; ${ }^{1} \mathrm{H}$ NMR (300 MHz, $\left.\mathrm{CDCl}_{3}\right) \delta 8.41(\mathrm{~s}, 1 \mathrm{H}), 7.80(\mathrm{~d}, J=7.3 \mathrm{~Hz}, 1 \mathrm{H}), 7.75-7.69(\mathrm{~m}, 1 \mathrm{H}), 7.36(\mathrm{~s}, 2 \mathrm{H}), 7.28(\mathrm{t}, J=$ $6.7 \mathrm{~Hz}, 2 \mathrm{H}), 7.15-7.08$ (m, 2H), 4.57 (d, $J=7.3 \mathrm{~Hz}, 1 \mathrm{H}), 3.76$ (s, 3H), 3.66 (d, $J=7.3 \mathrm{~Hz}$, $1 \mathrm{H}), 3.45$ (s, 3H), 3.05 (s, 3H), $2.79(\mathrm{~s}, 3 \mathrm{H}) .{ }^{13} \mathrm{C} \mathrm{NMR}\left(75 \mathrm{MHz}, \mathrm{CDCl}_{3}\right) \delta 169.1,168.0$, 136.7, 132.8, 131.9, 129.1, 128.4, 128.3, 127.2, 126.5, 122.5, 120.6, 120.1, 120.1, 111.3, 111.1, 102.3, 52.5, 52.3, 51.9, 50.5, 49.6, 39.0. HRMS (ESI): Calc. for $\mathrm{C}_{22} \mathrm{H}_{19} \mathrm{NO}_{5}(\mathrm{M}+\mathrm{H}-$ $\mathrm{MeOH})^{+}: 378.1336$ found: 378.1335 .

\section{Copies of ${ }^{1} \mathrm{H}$ and ${ }^{13} \mathrm{C}$ NMR spectra}




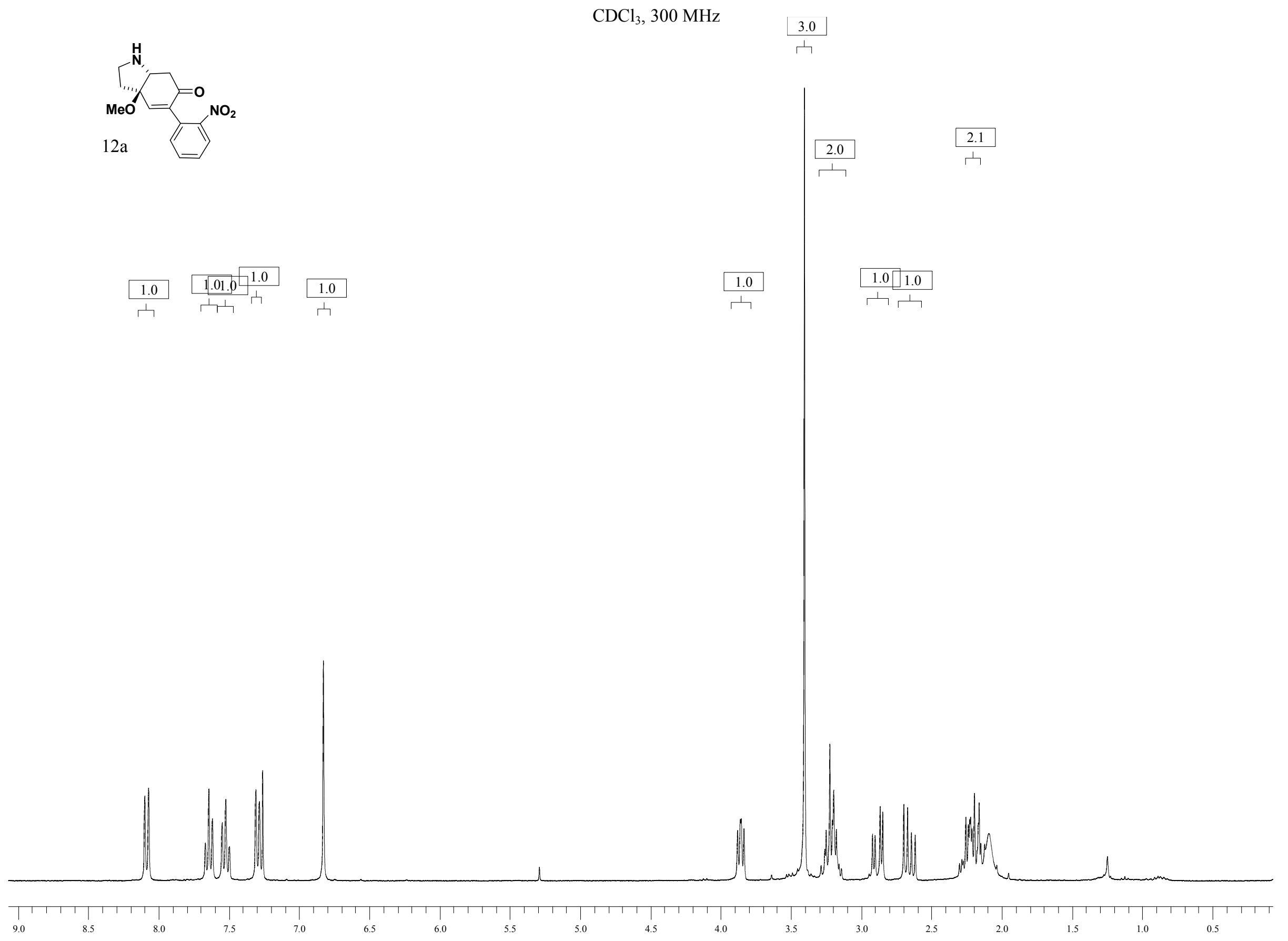




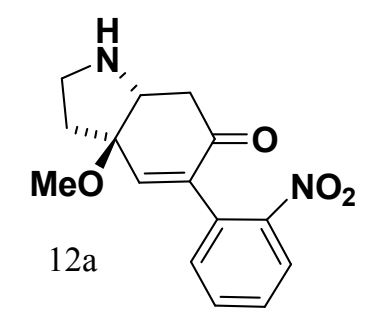

$\mathrm{CDCl}_{3}, 75 \mathrm{MHz}$

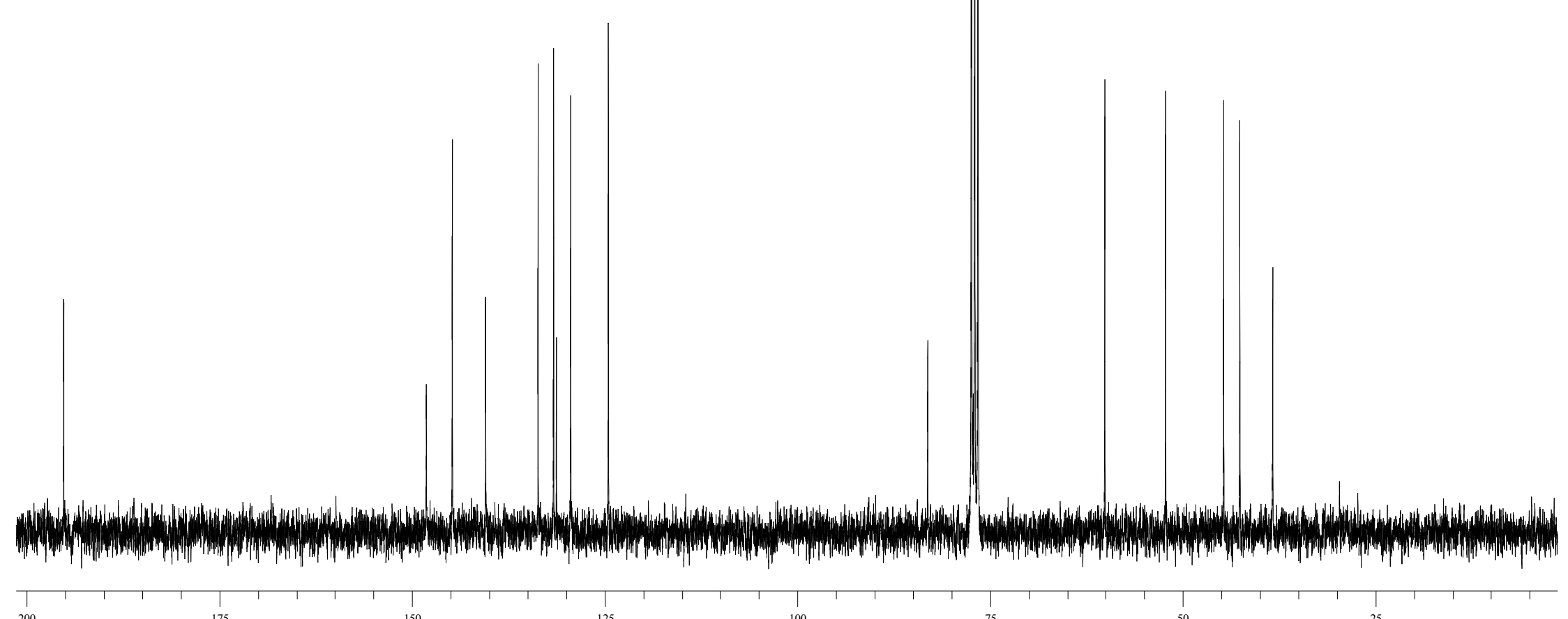




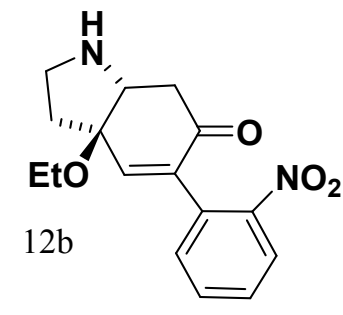

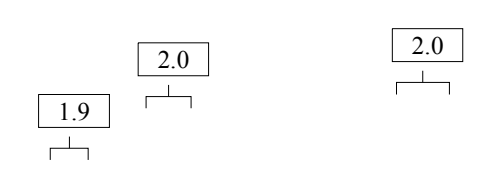

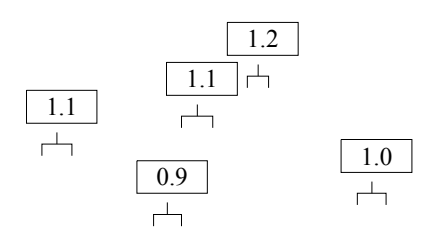



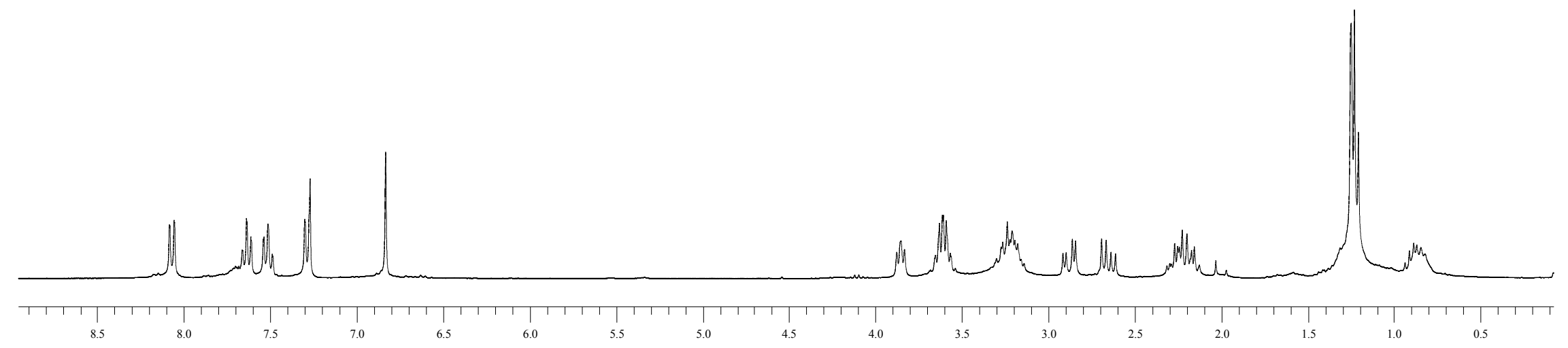




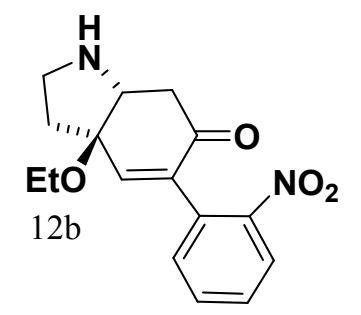

$\mathrm{CDCl}_{3}, 75 \mathrm{MHz}$

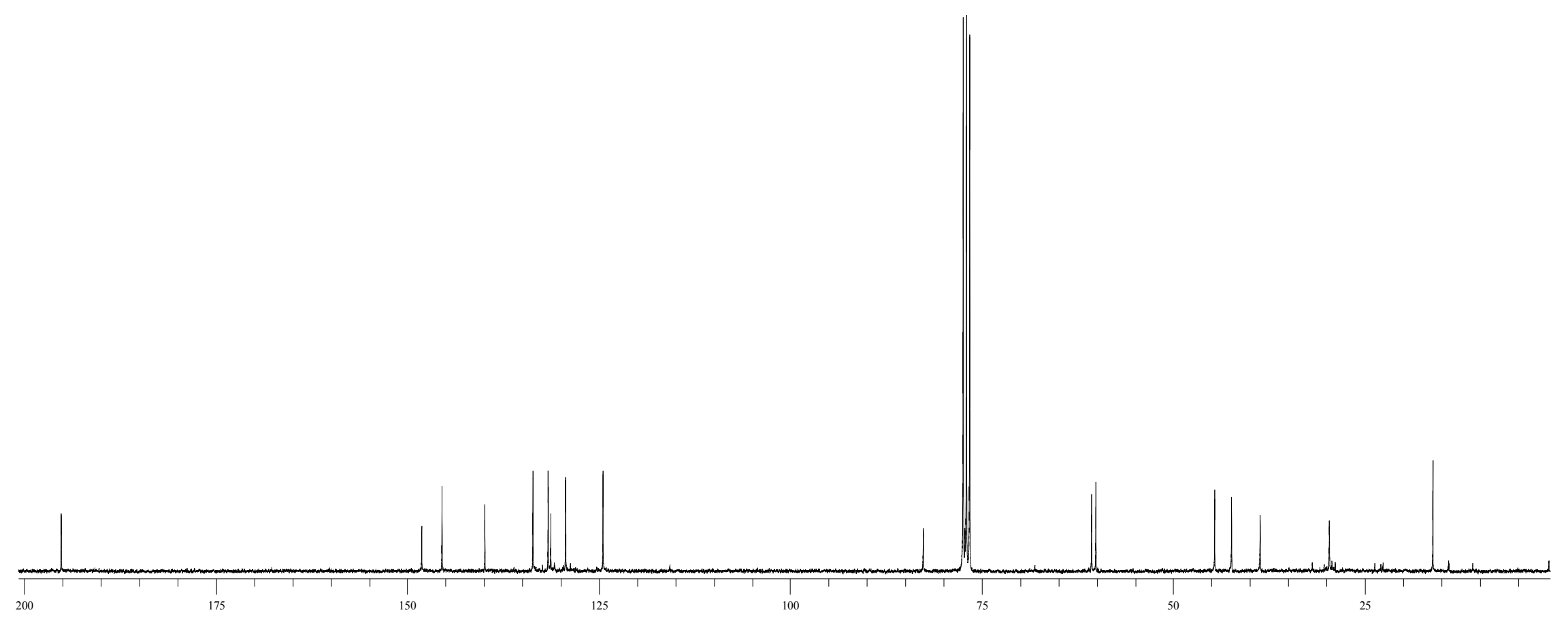




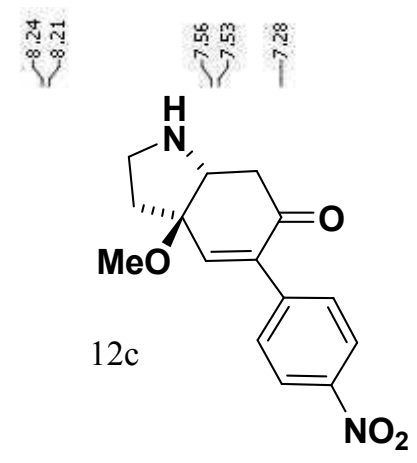

$\mathrm{CDCl}_{3}, 300 \mathrm{MHz}$

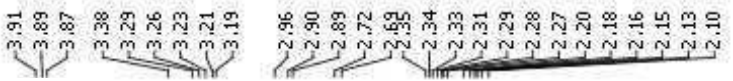

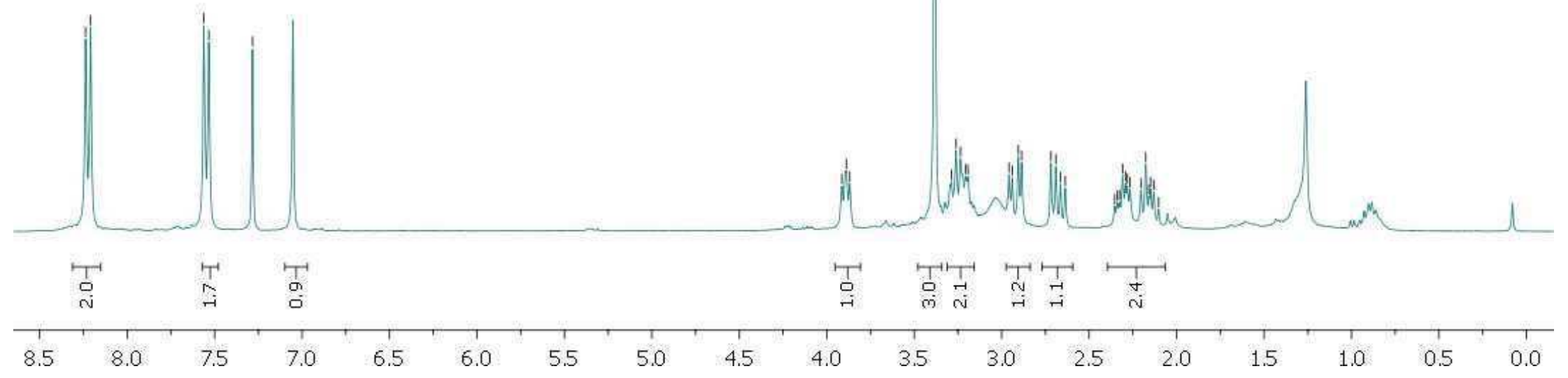




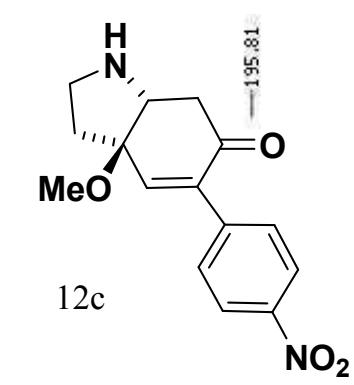

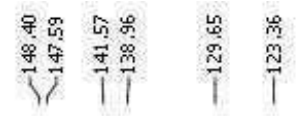

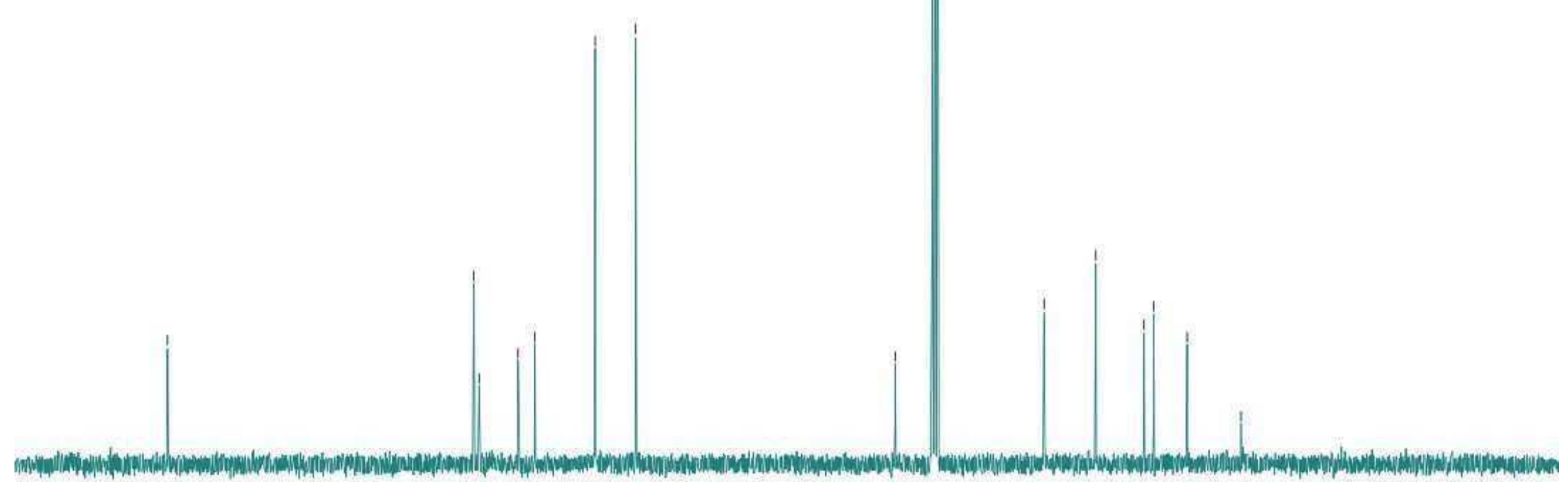

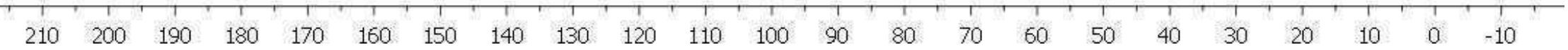




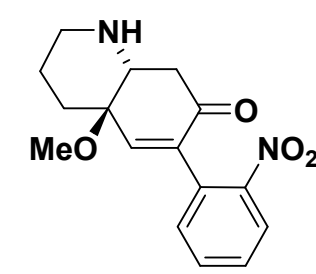

$12 d$

\section{$\mathrm{CDCl}_{3}, 300 \mathrm{MHz}$}

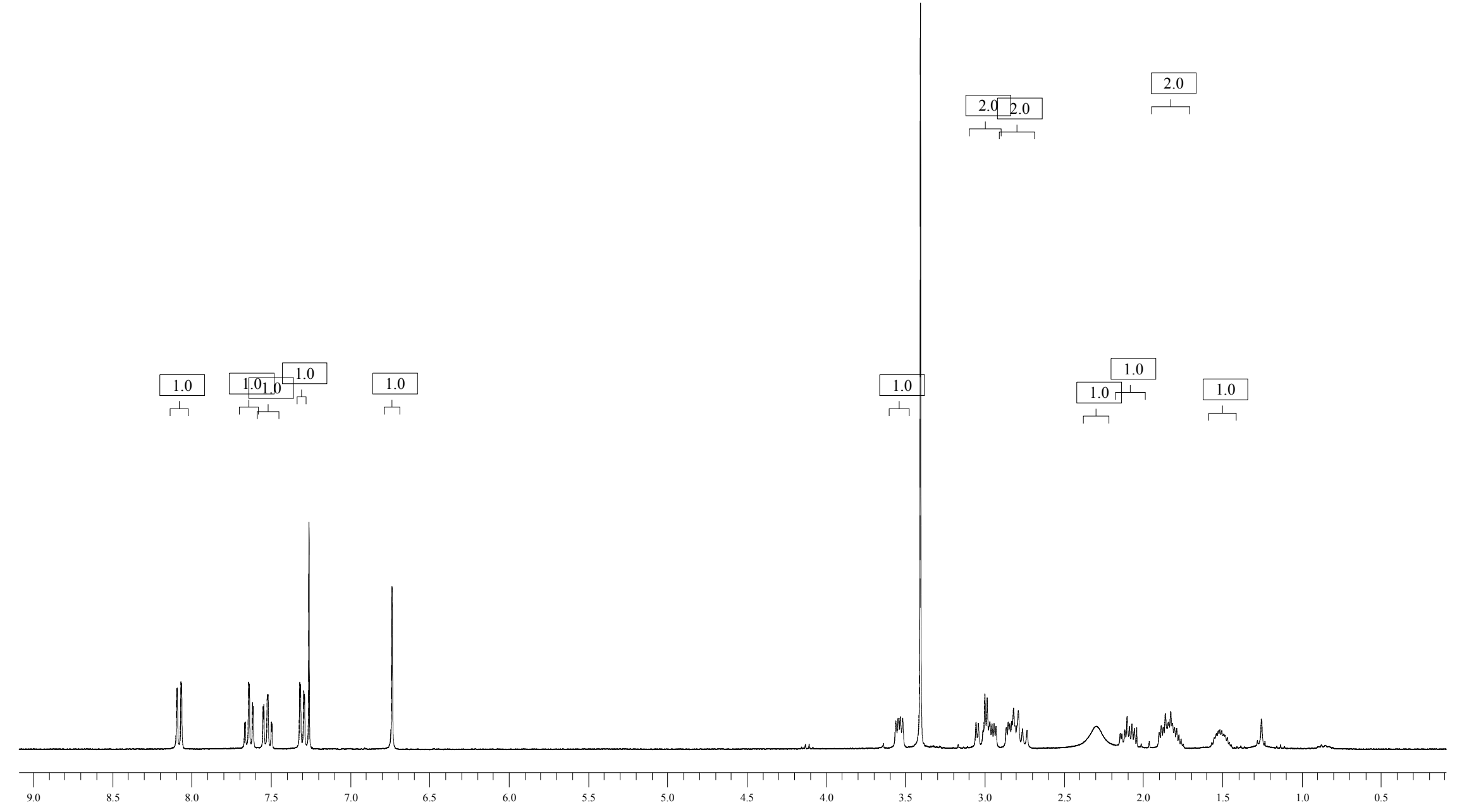


$\mathrm{CDCl}_{3}, 75 \mathrm{MHz}$

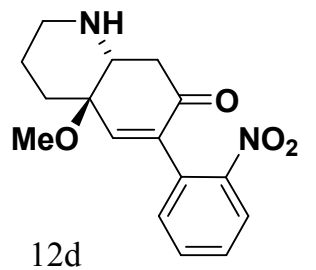

$12 d$

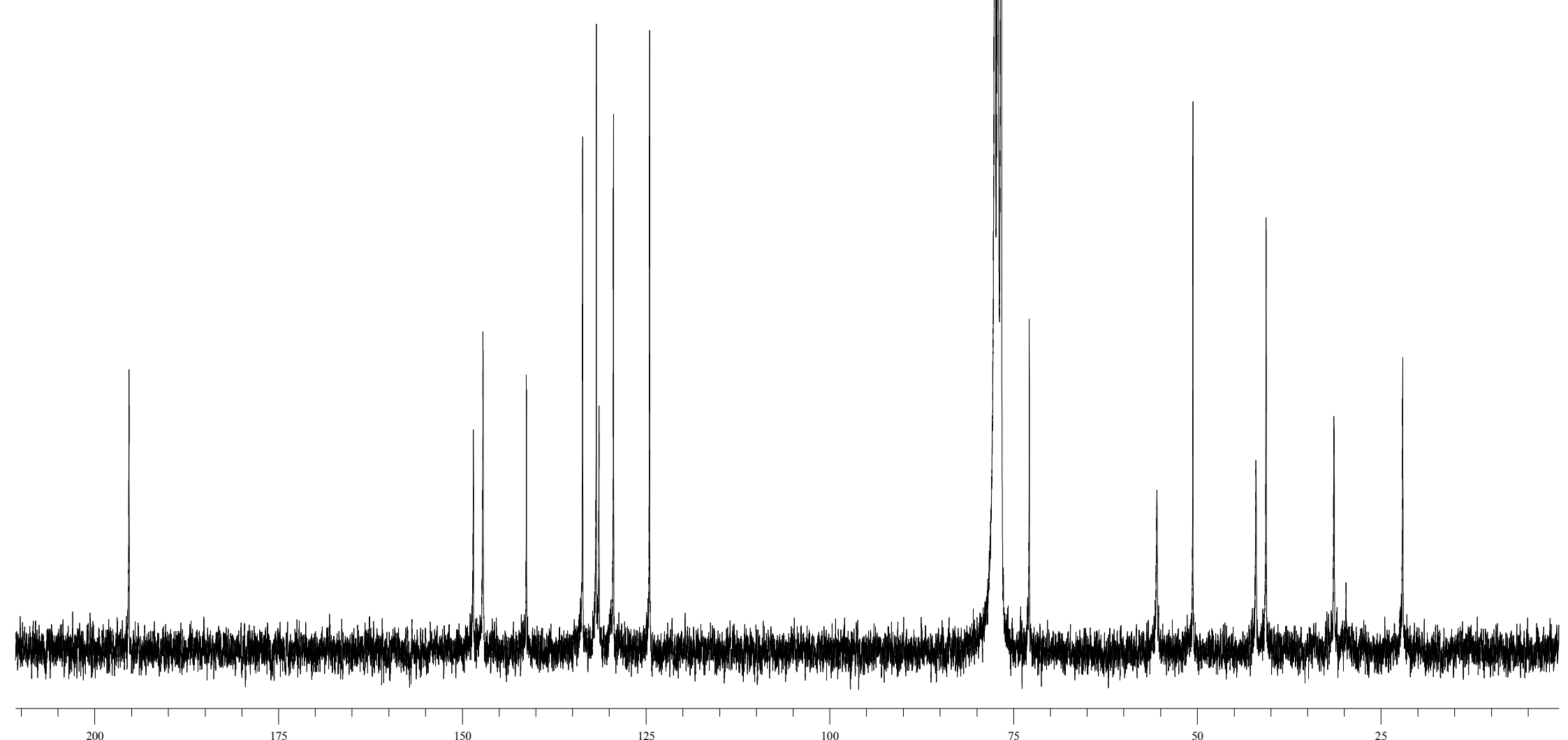




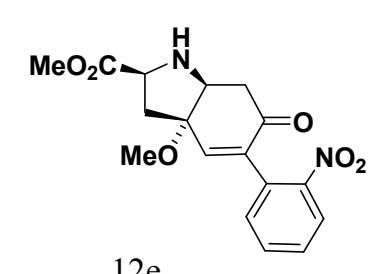

$12 \mathrm{e}$

$$
\begin{array}{|l|l|}
\hline 3.0 & 3.0 \\
\hline & +
\end{array}
$$

$\mathrm{CDCl}_{3}, 300 \mathrm{MHz}$

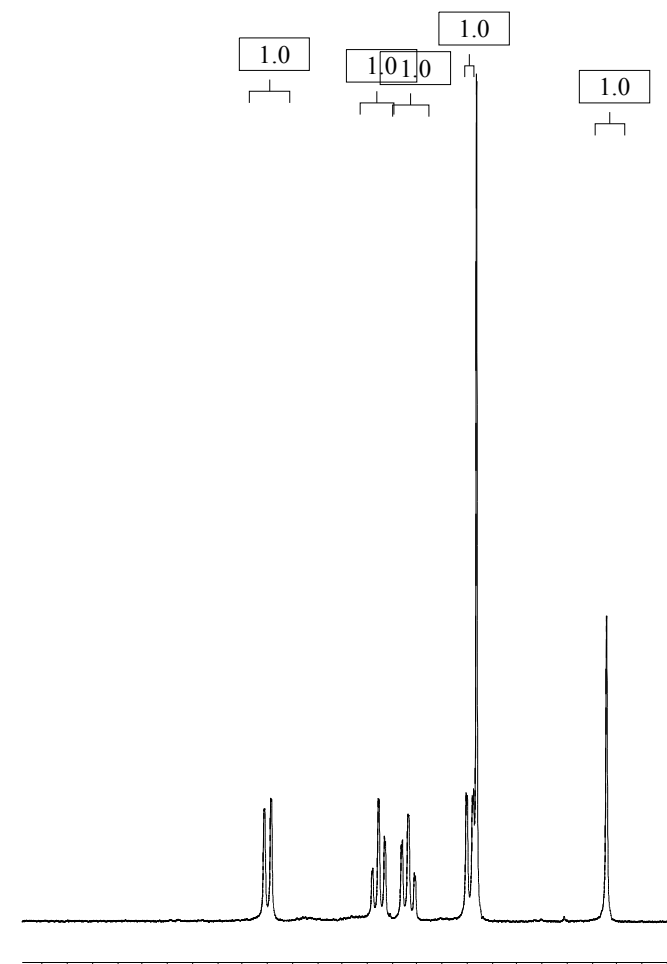

$\prod_{9.0}$ 


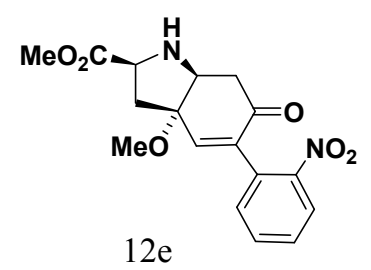

$\mathrm{CDCl}_{3}, 75 \mathrm{MHz}$

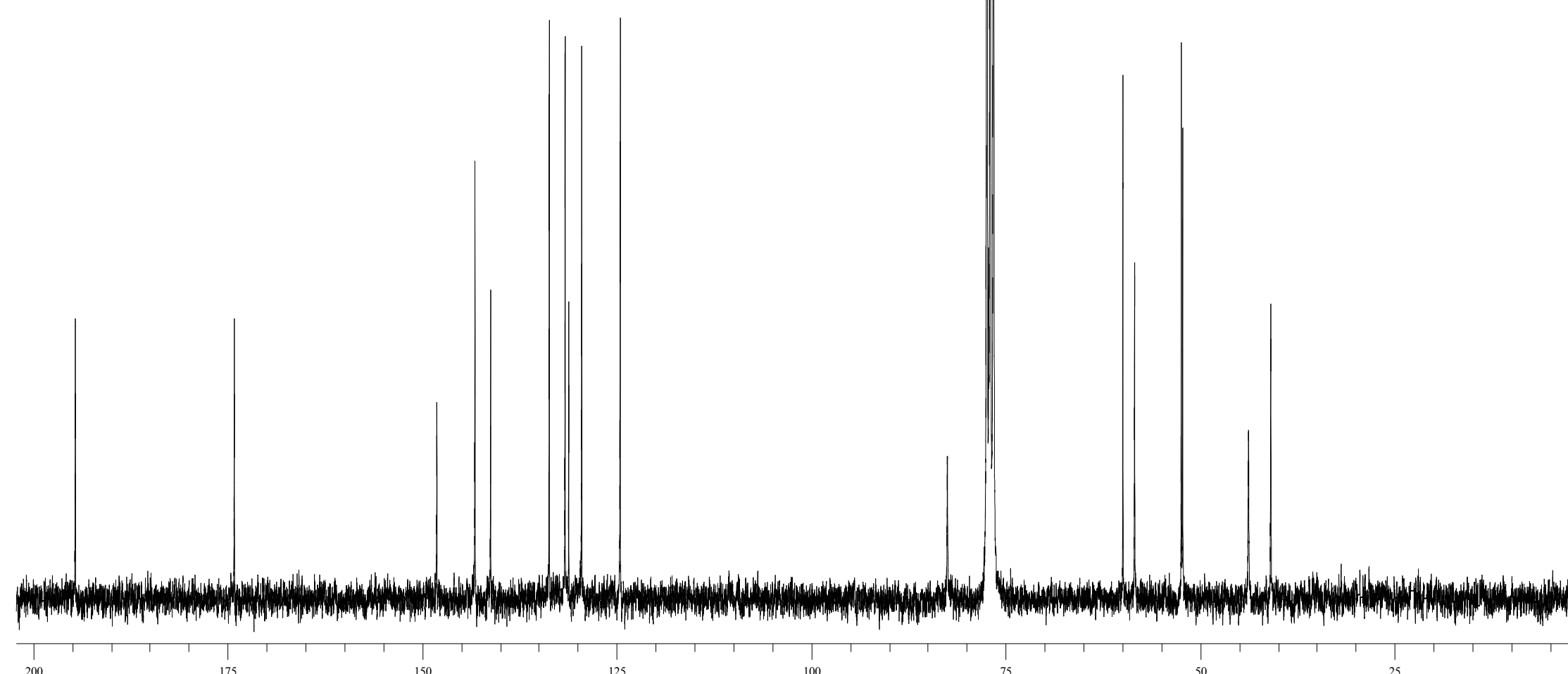


$\mathrm{CDCl}_{3}, 300 \mathrm{MHz}$



$12 \mathrm{f}$
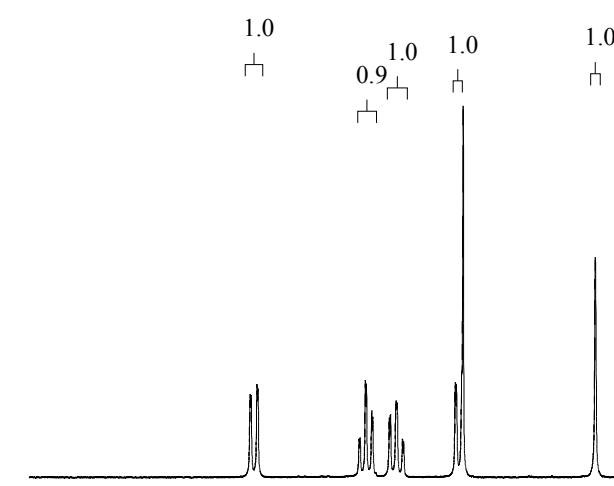

3.1

ウ
3.0

ウ

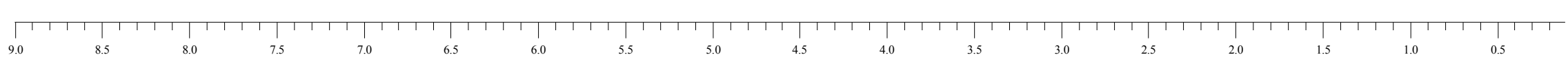

S23 




$12 \mathrm{f}$
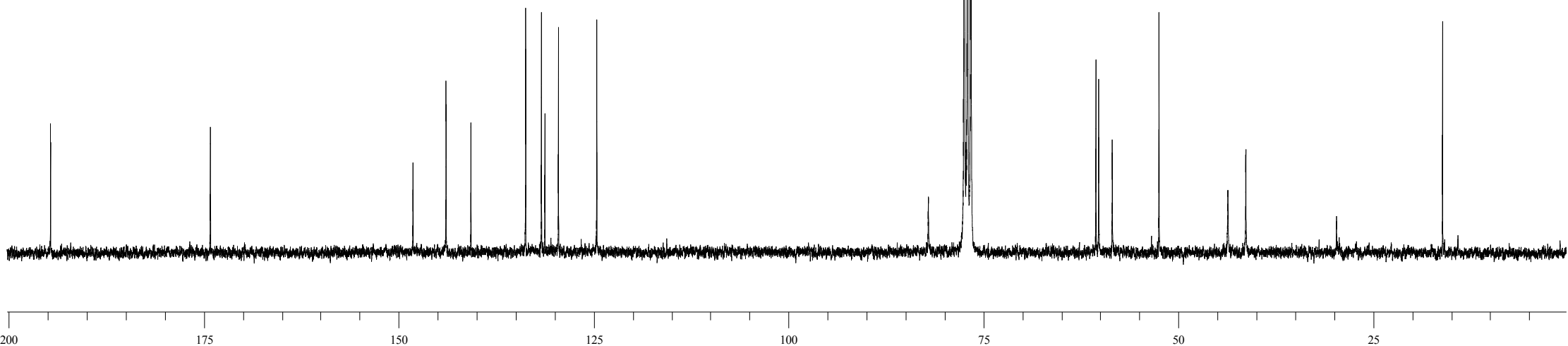


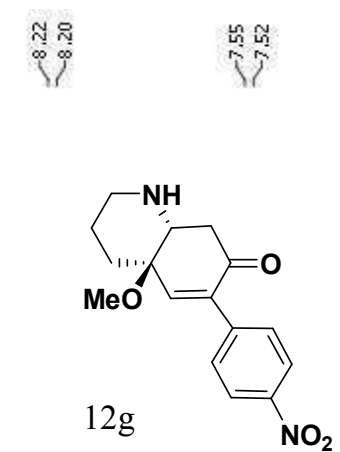

$\mathrm{CDCl}_{3}, 300 \mathrm{MHz}$

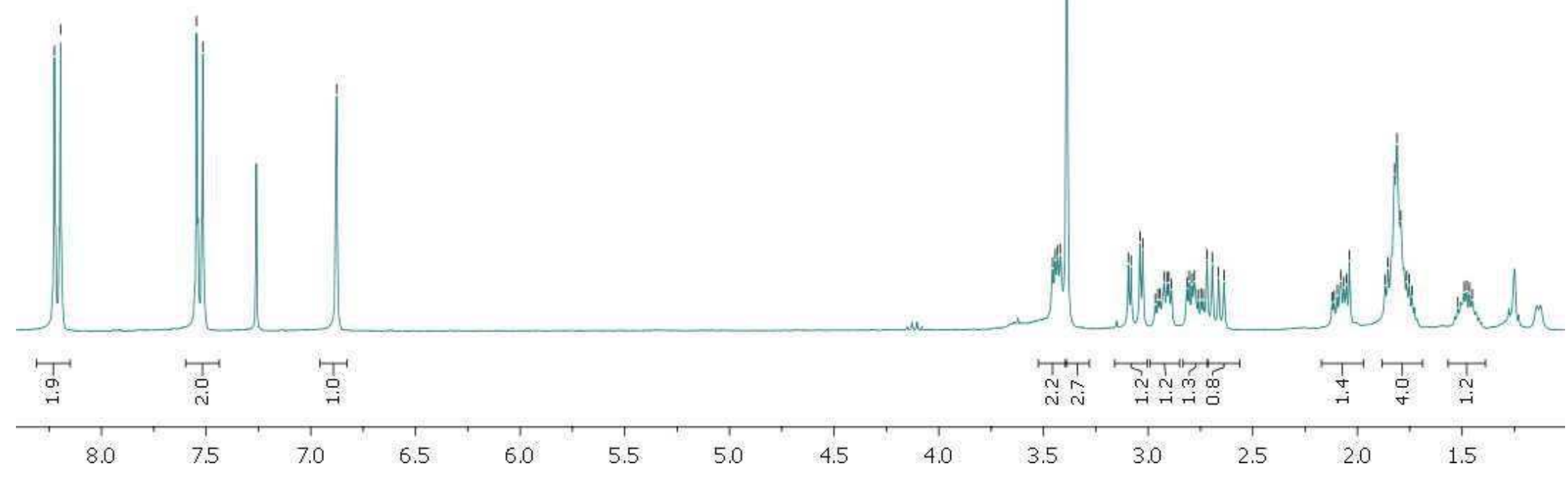






$$
\text { 宁 }
$$
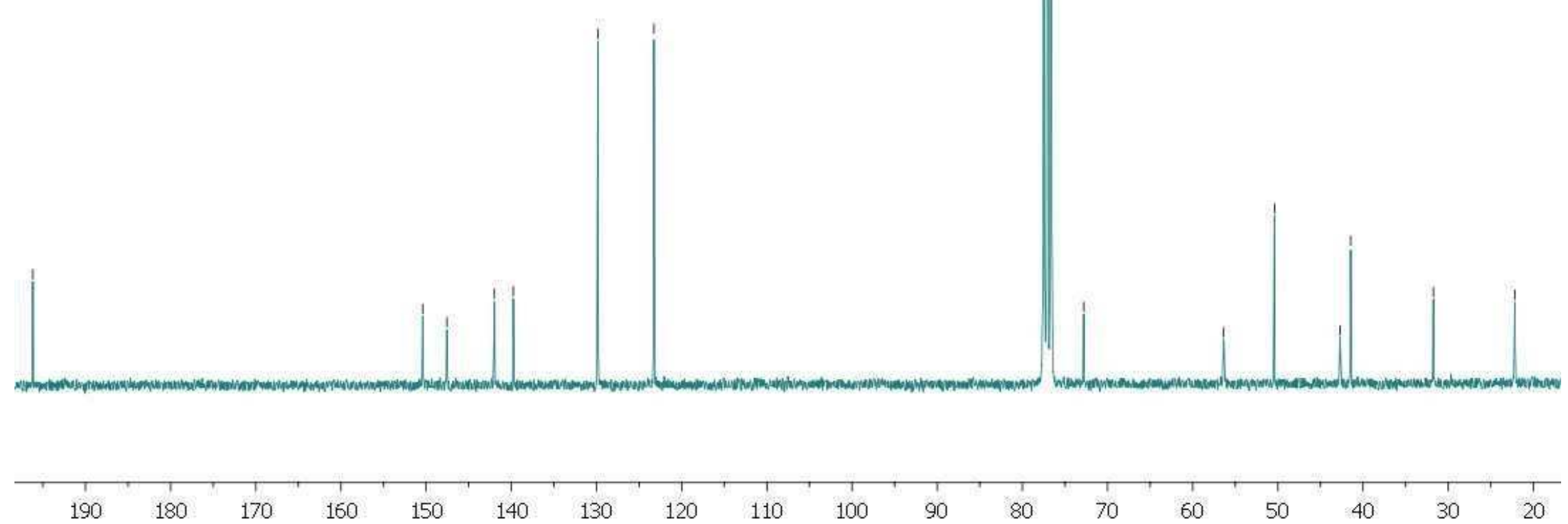


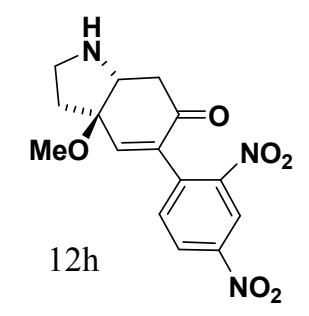

$\mathrm{CDCl}_{3}, 300 \mathrm{MHz}$

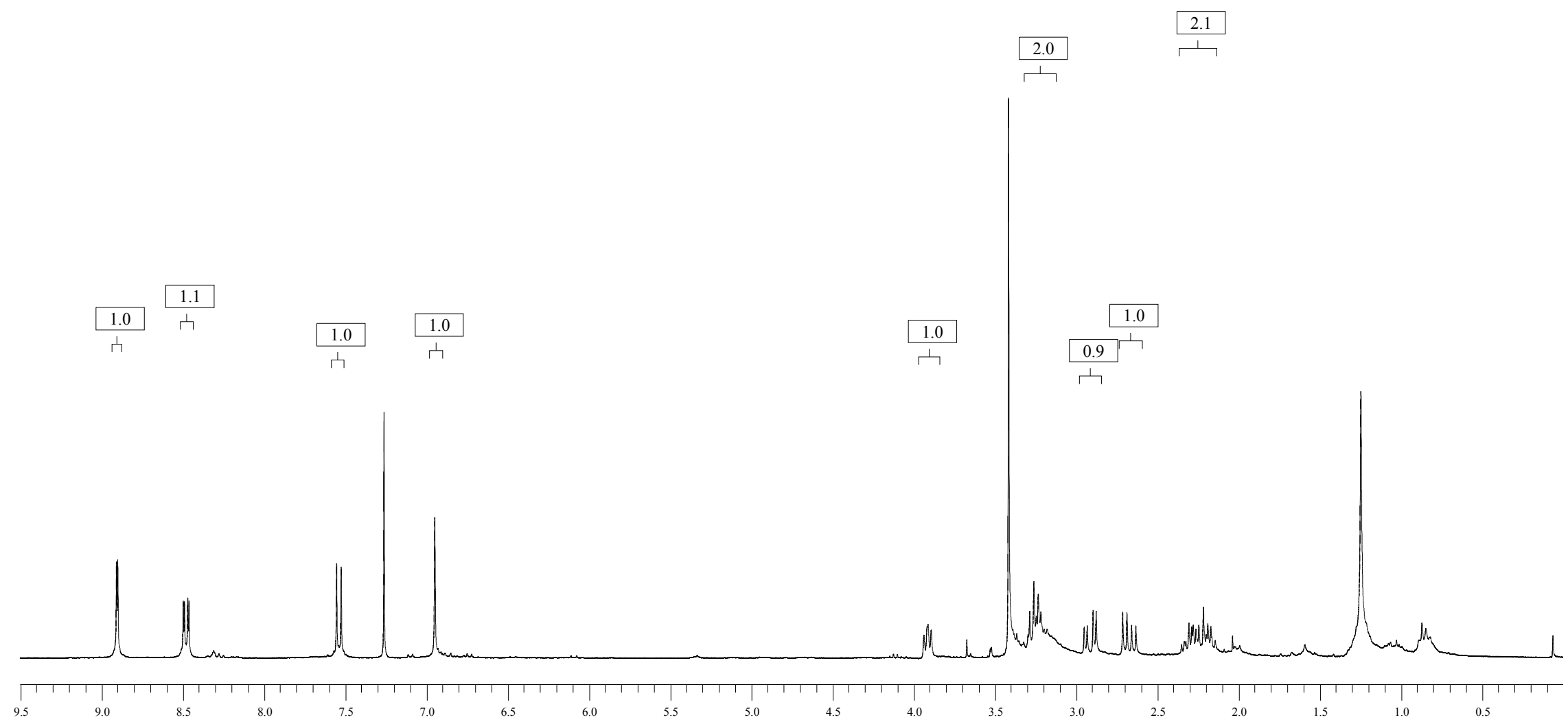


$\mathrm{CDCl}_{3}, 75 \mathrm{MHz}$

$\mathrm{CDCl}_{3}, 300 \mathrm{MHz} \quad \stackrel{3.0}{\dot{1}}$

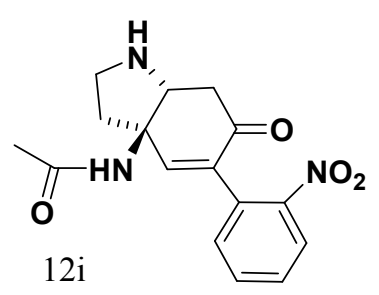

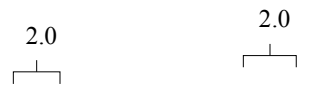

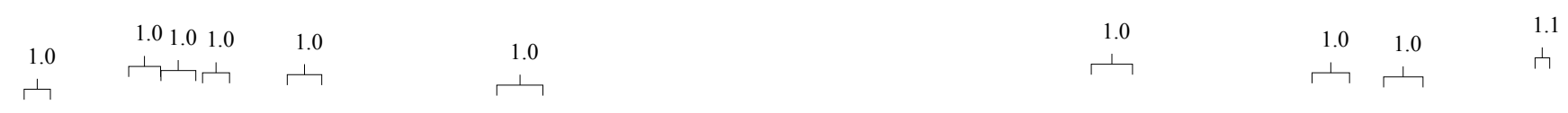

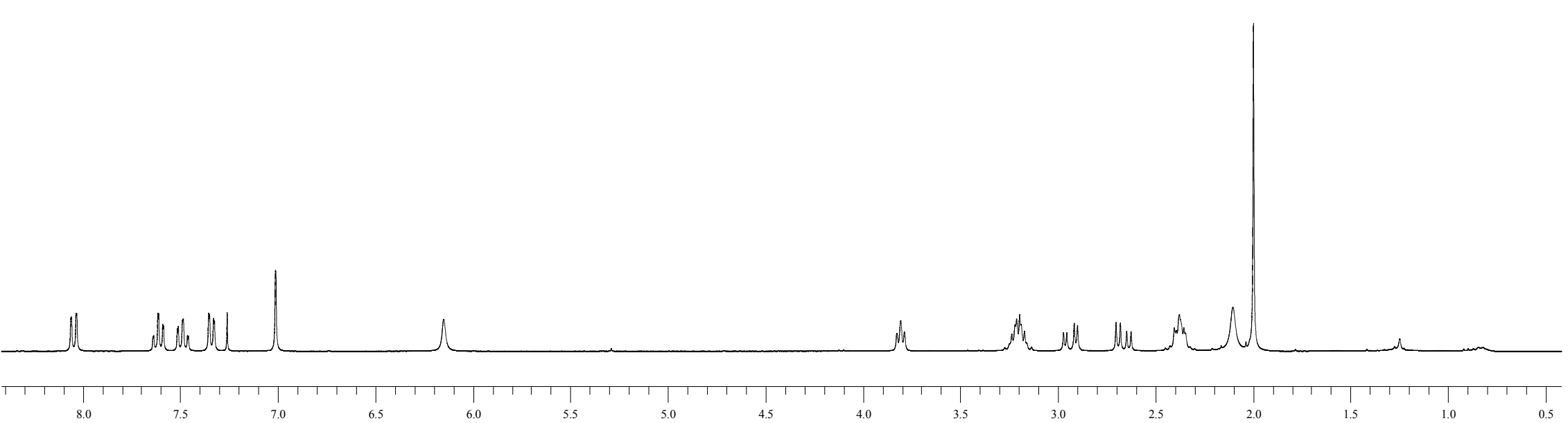




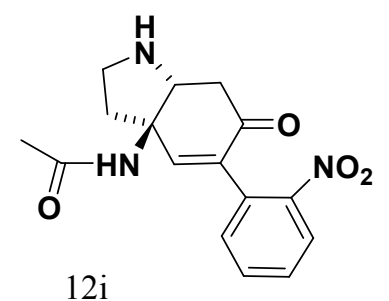

$\mathrm{CDCl}_{3}, 75 \mathrm{MHz}$

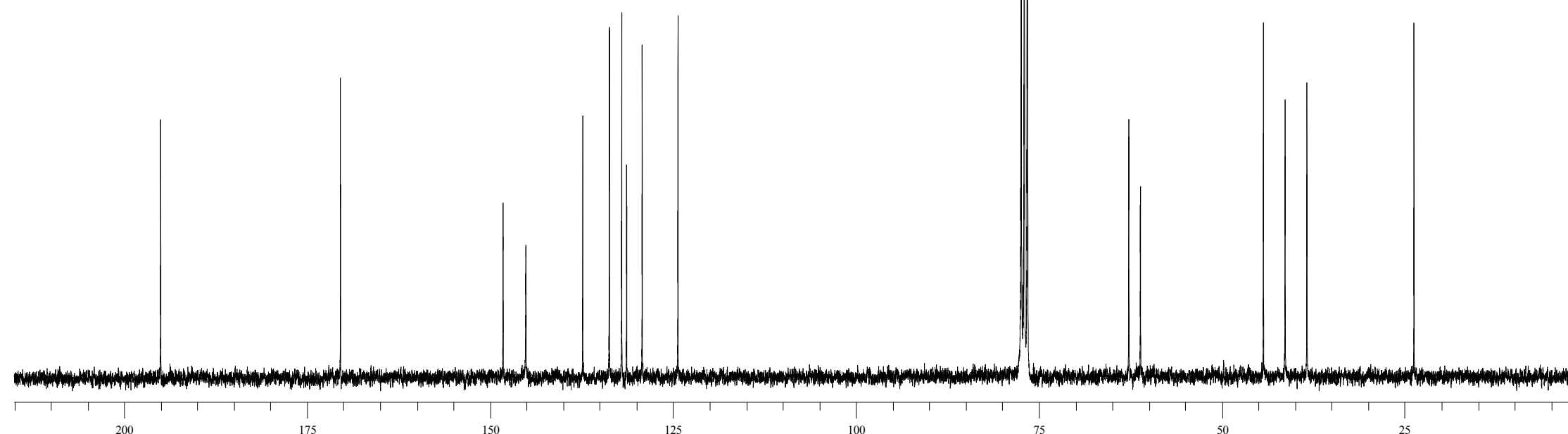




$\mathrm{CDCl}_{3}, 75 \mathrm{MHz}$
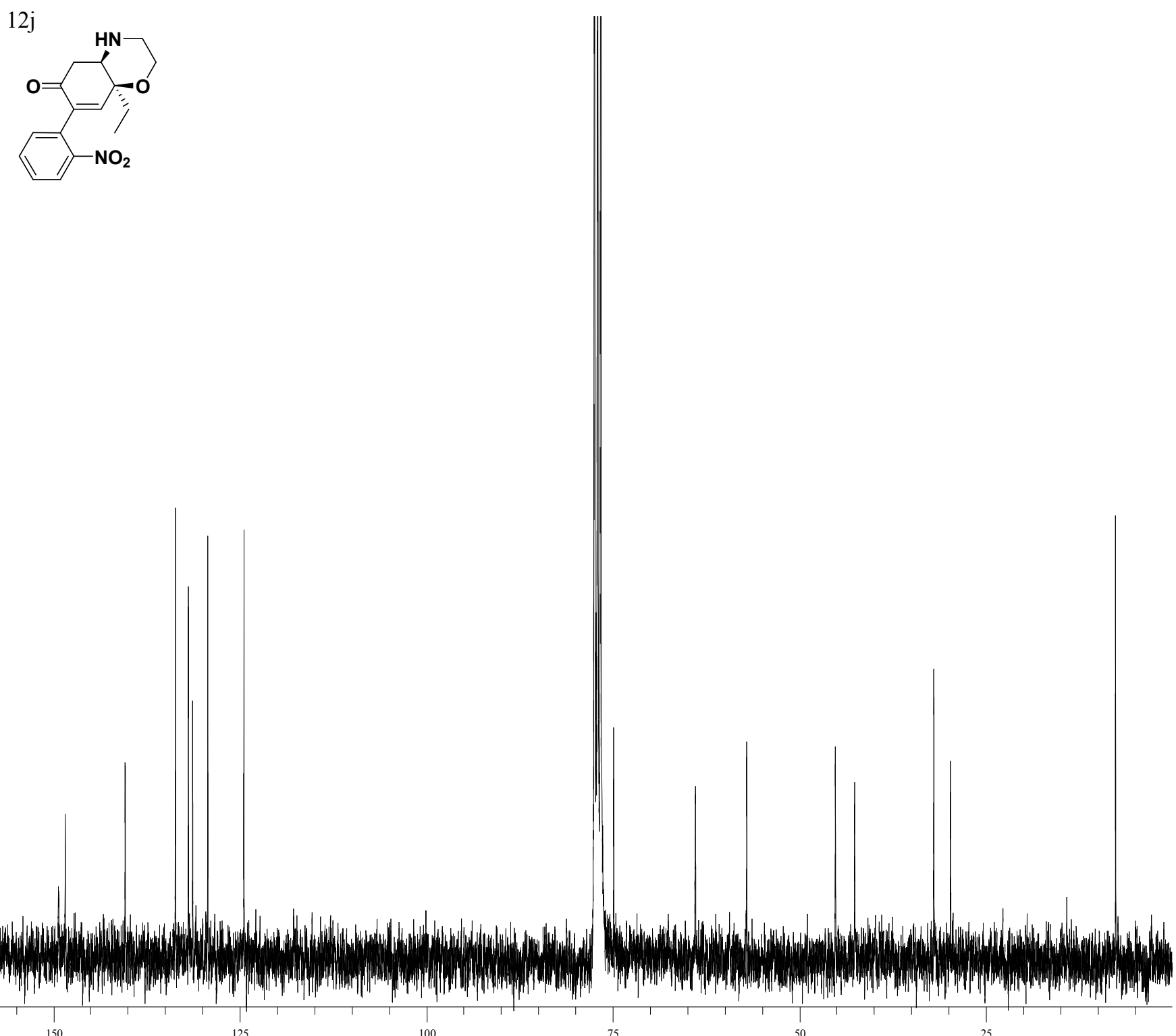




$12 \mathrm{k}$

$\mathrm{CDCl}_{3}, 300 \mathrm{MHz}$

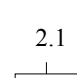

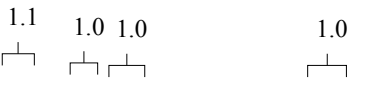

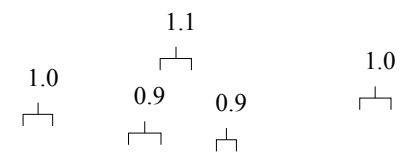

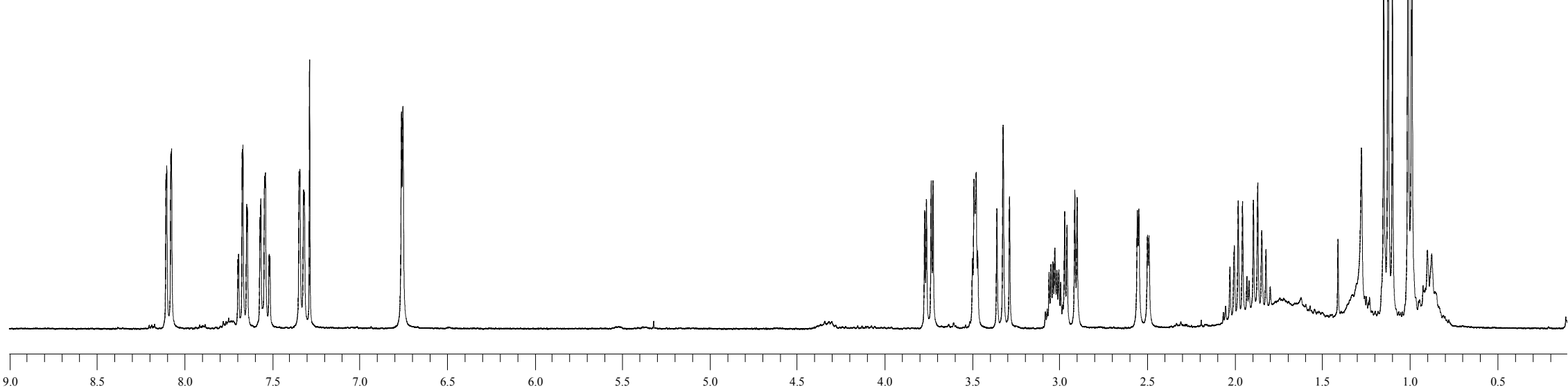




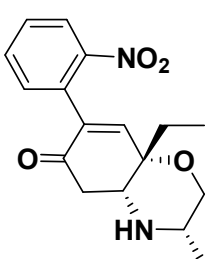

$\mathrm{CDCl}_{3}, 75 \mathrm{MHz}$

$12 \mathrm{k}$ "

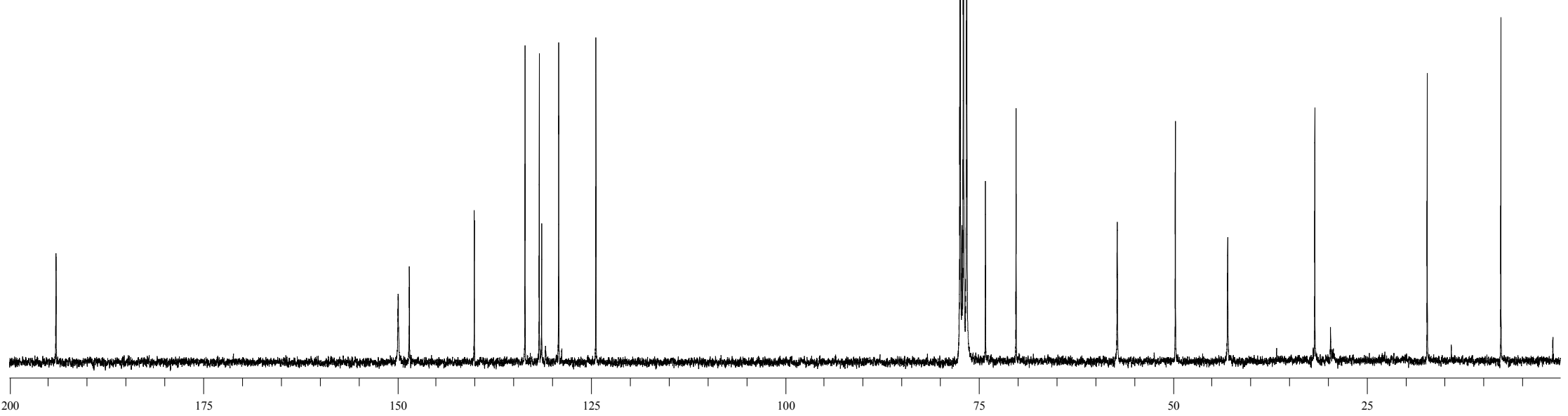




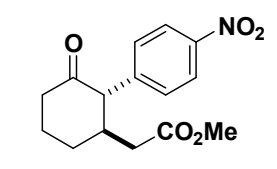

15

$\mathrm{CDCl}_{3}, 300 \mathrm{MHz}$

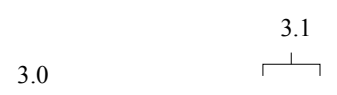



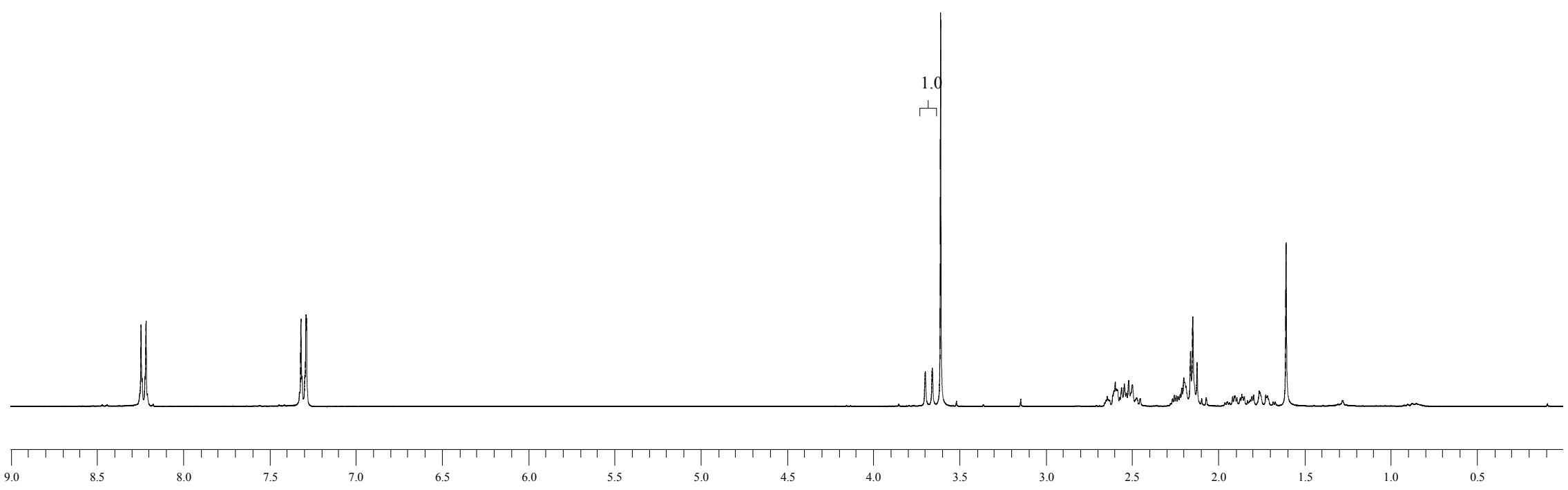




$$
\cdots
$$




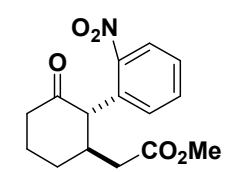

17
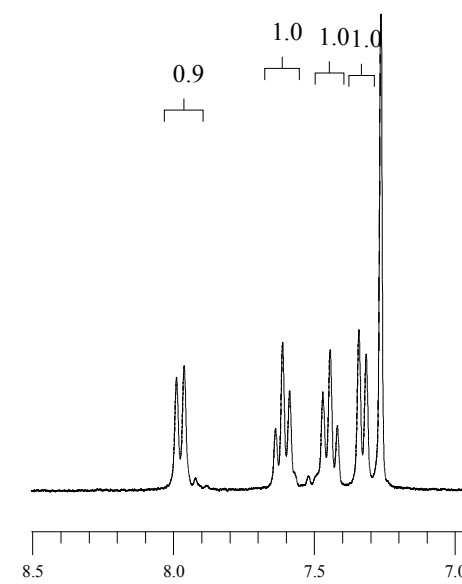

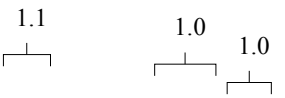

0.9




I I

1 


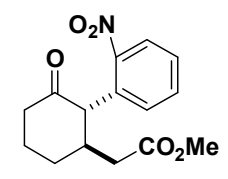

17

(1) 200 

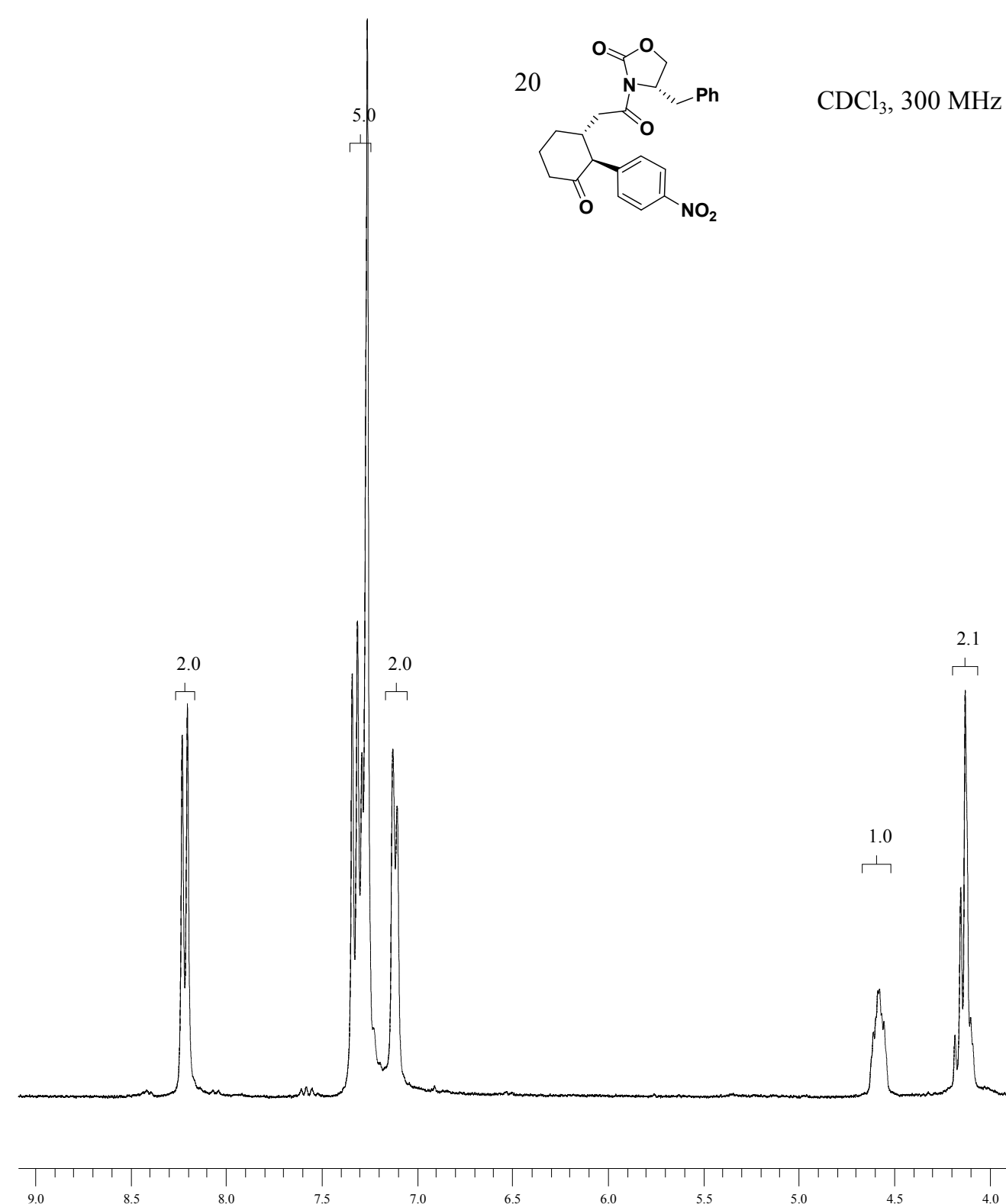

2.1

2.0

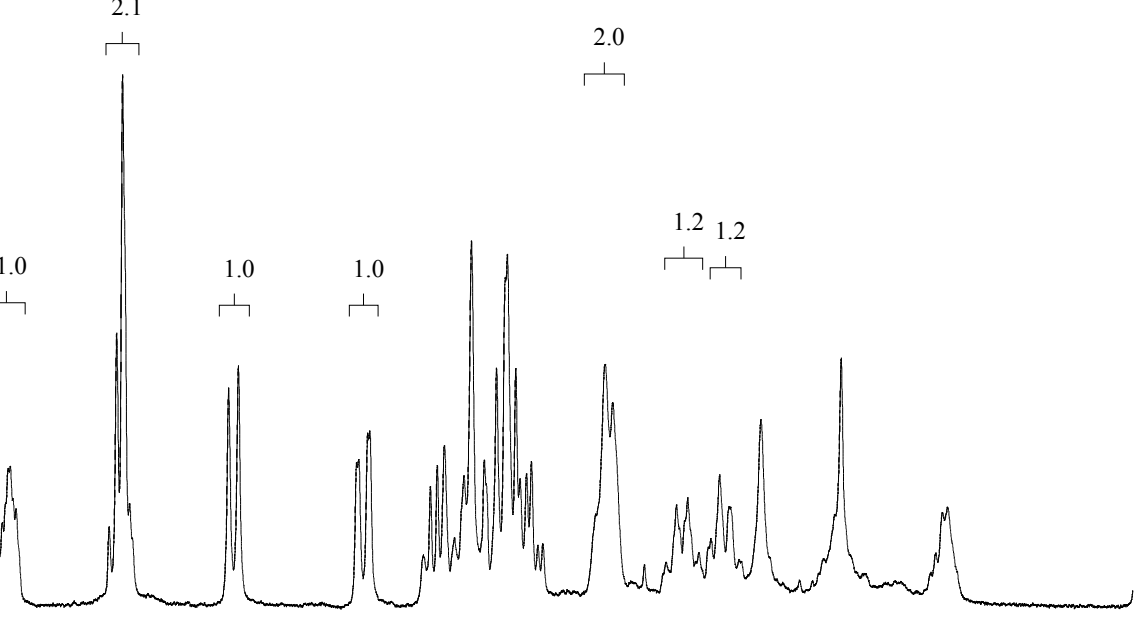





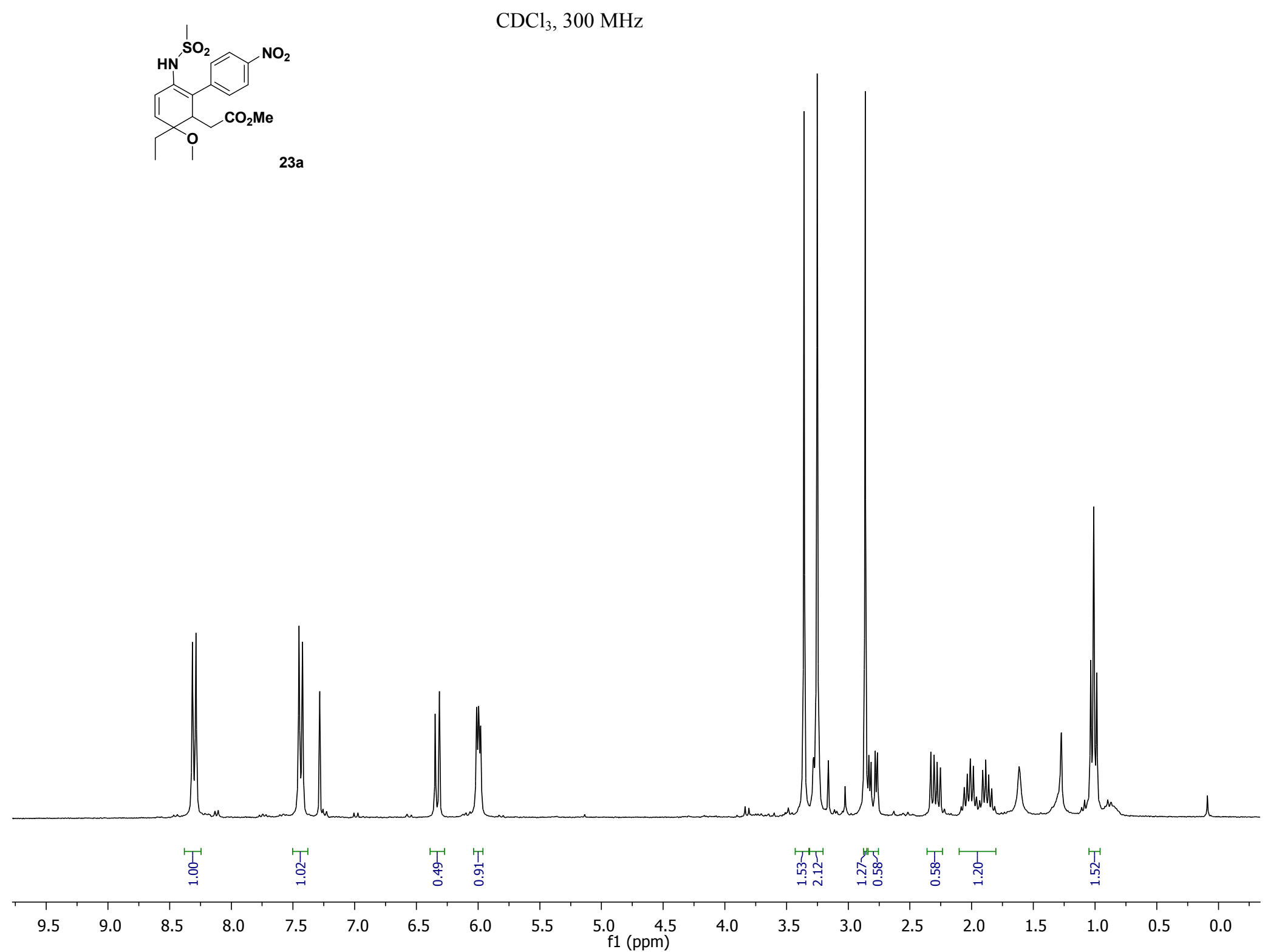


$\mathrm{CDCl}_{3}, 75 \mathrm{MHz}$
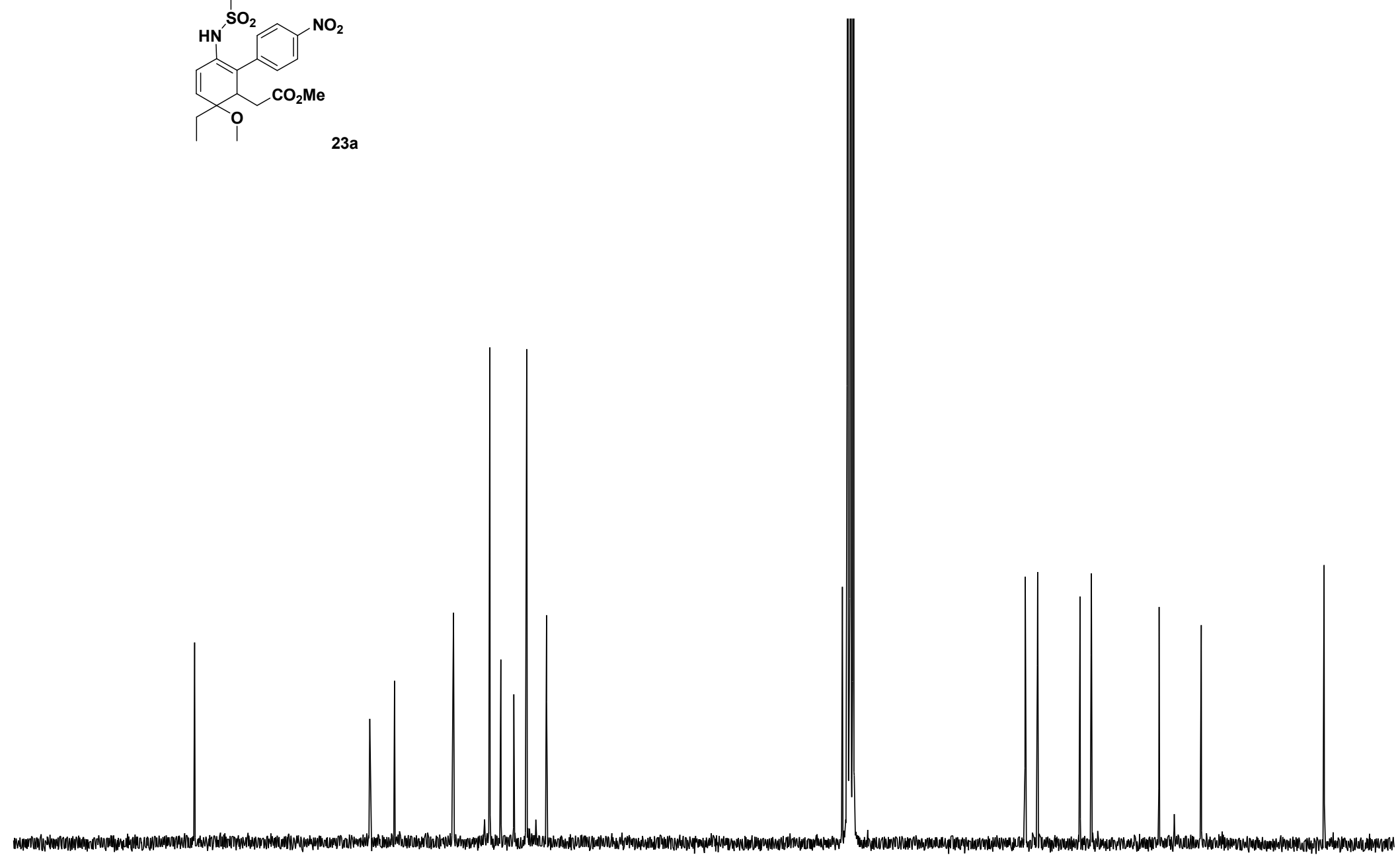

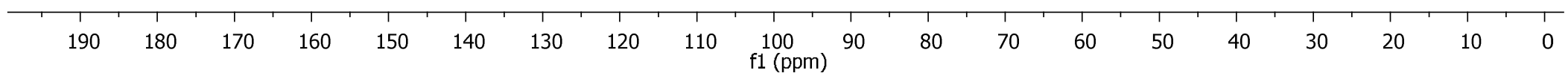




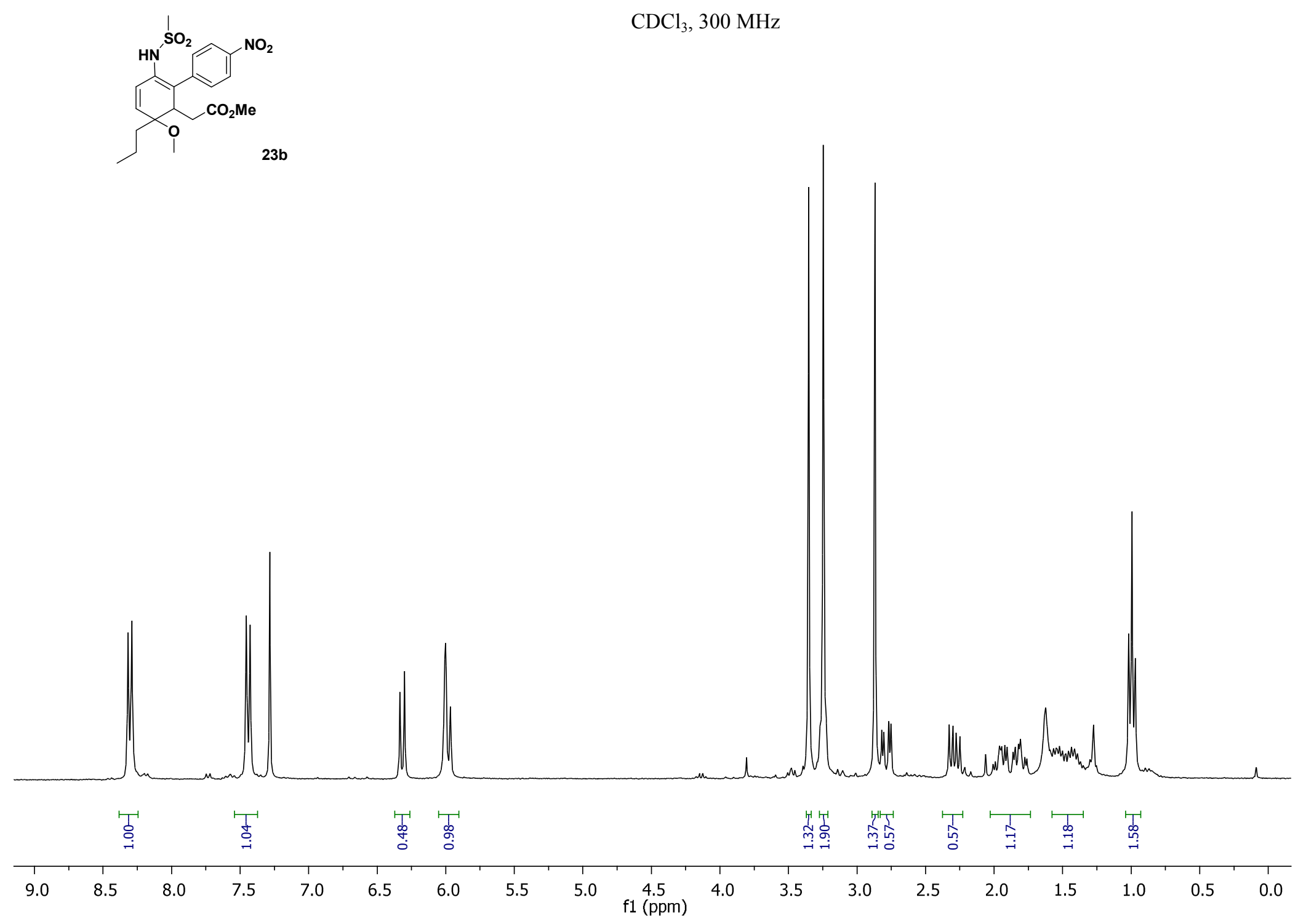




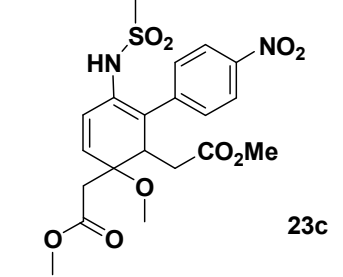

$\mathrm{CDCl}_{3}, 300 \mathrm{MHz}$

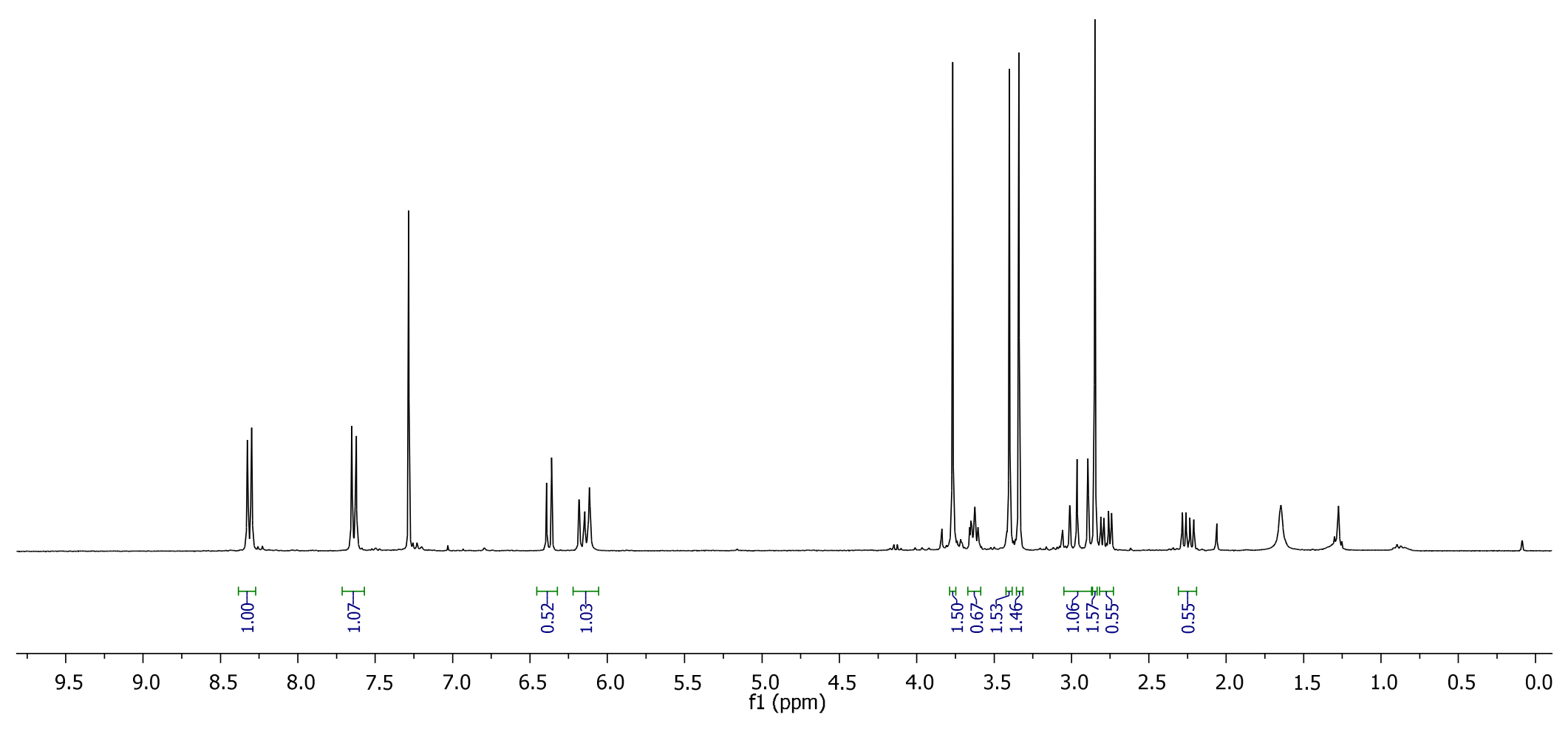


$\mathrm{CDCl}_{3}, 75 \mathrm{MHz}$
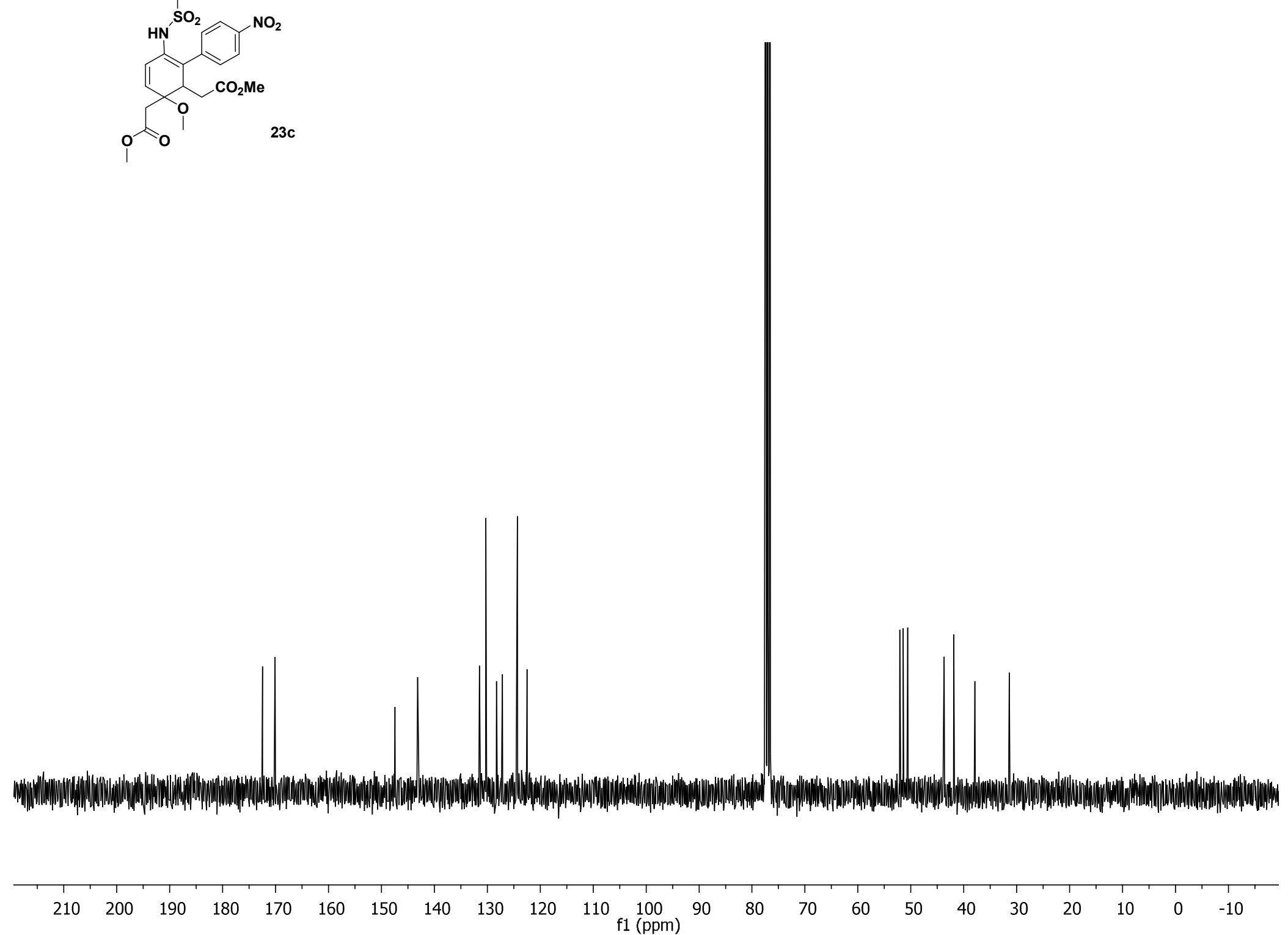

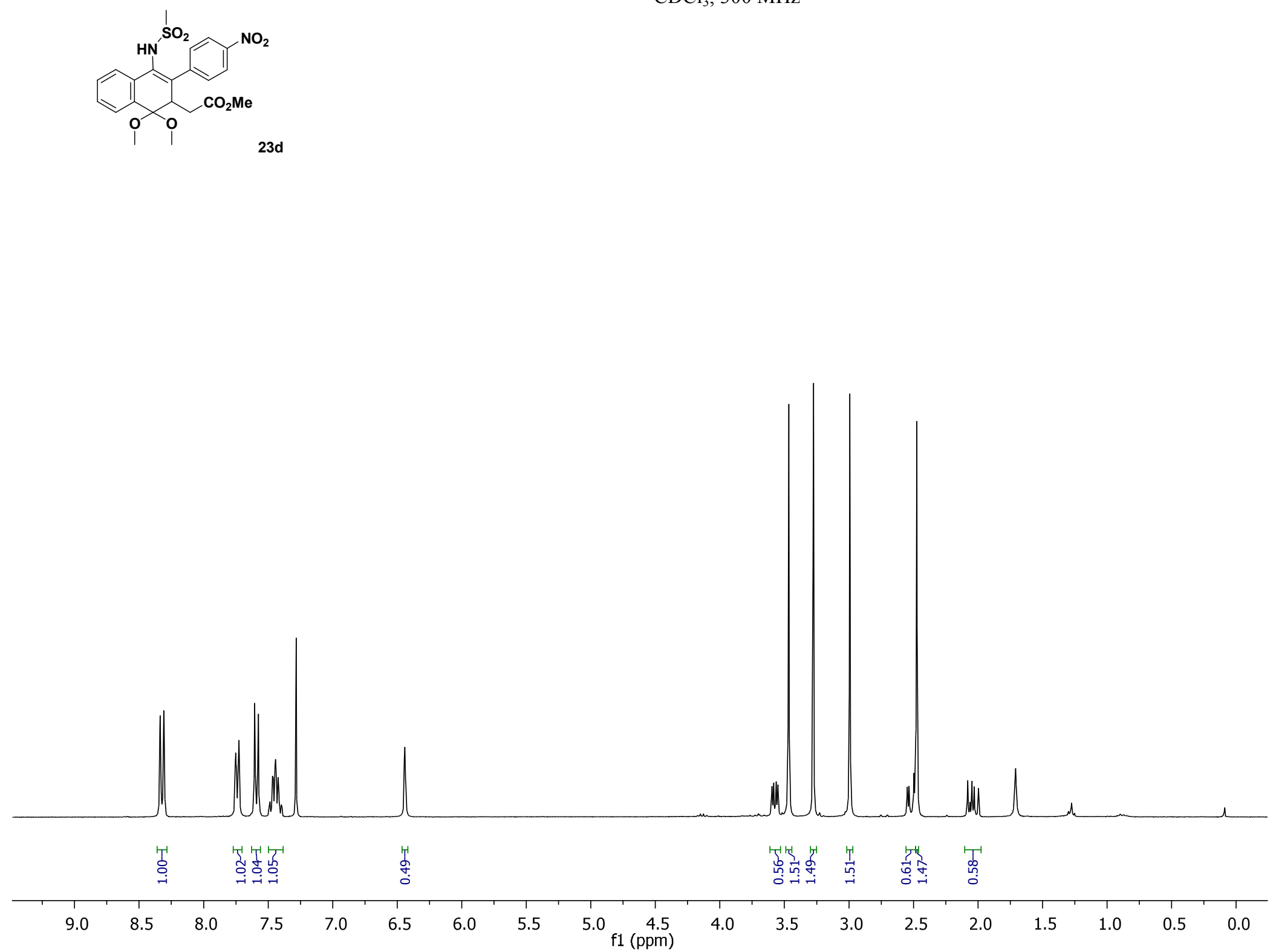
$\mathrm{CDCl}_{3}, 75 \mathrm{MHz}$
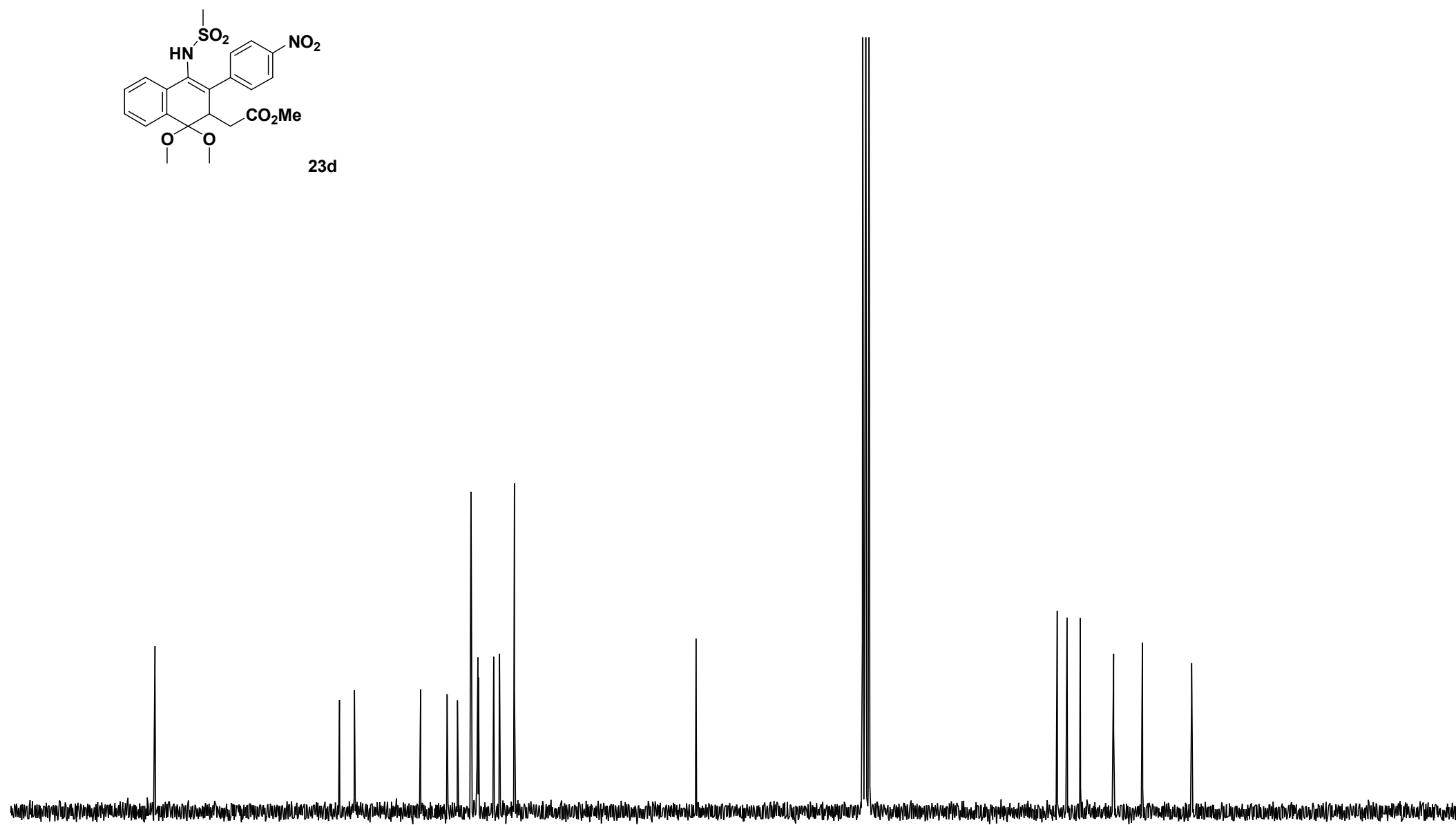









$\mathrm{CDCl}_{3}, 75 \mathrm{MHz}$
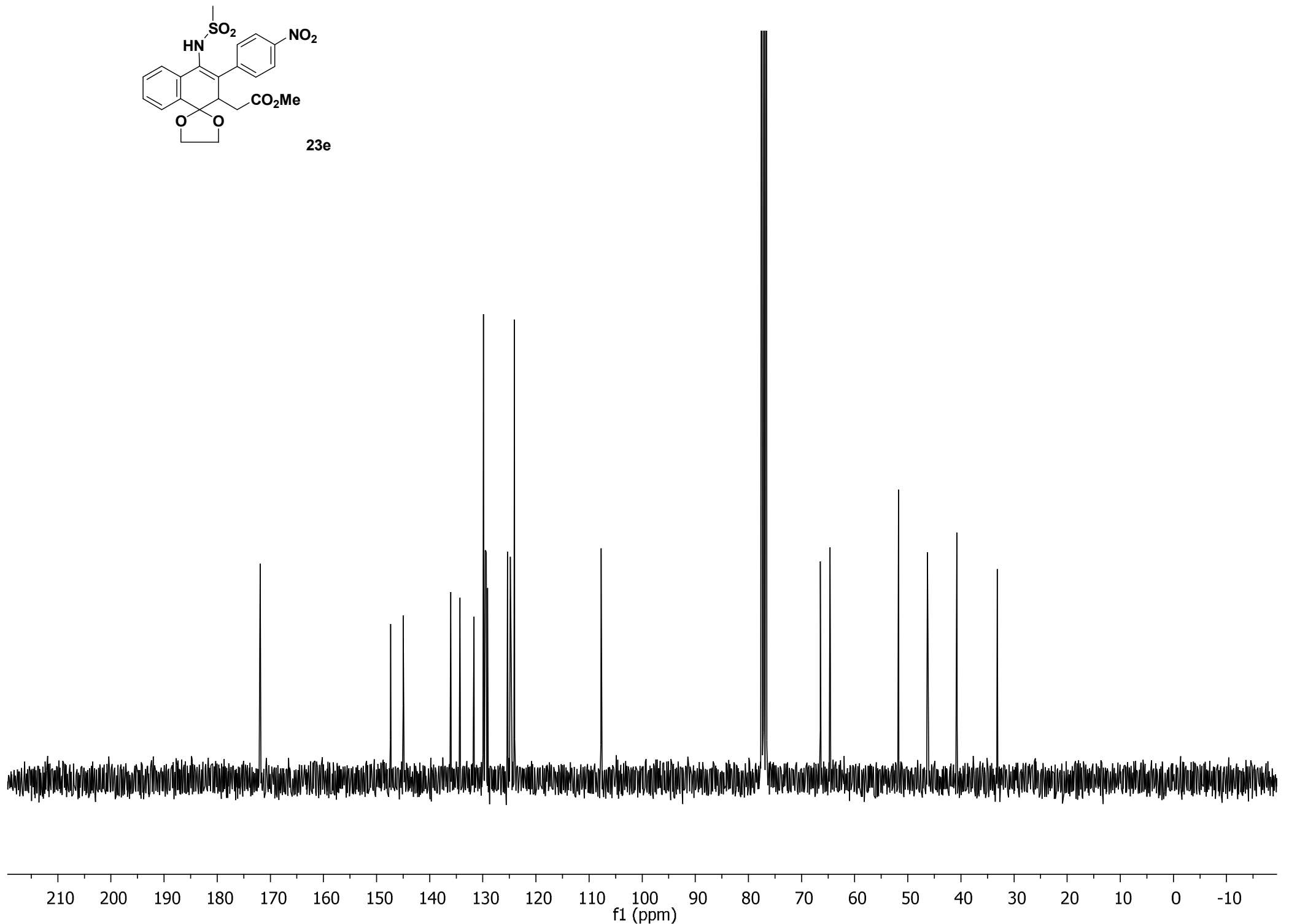

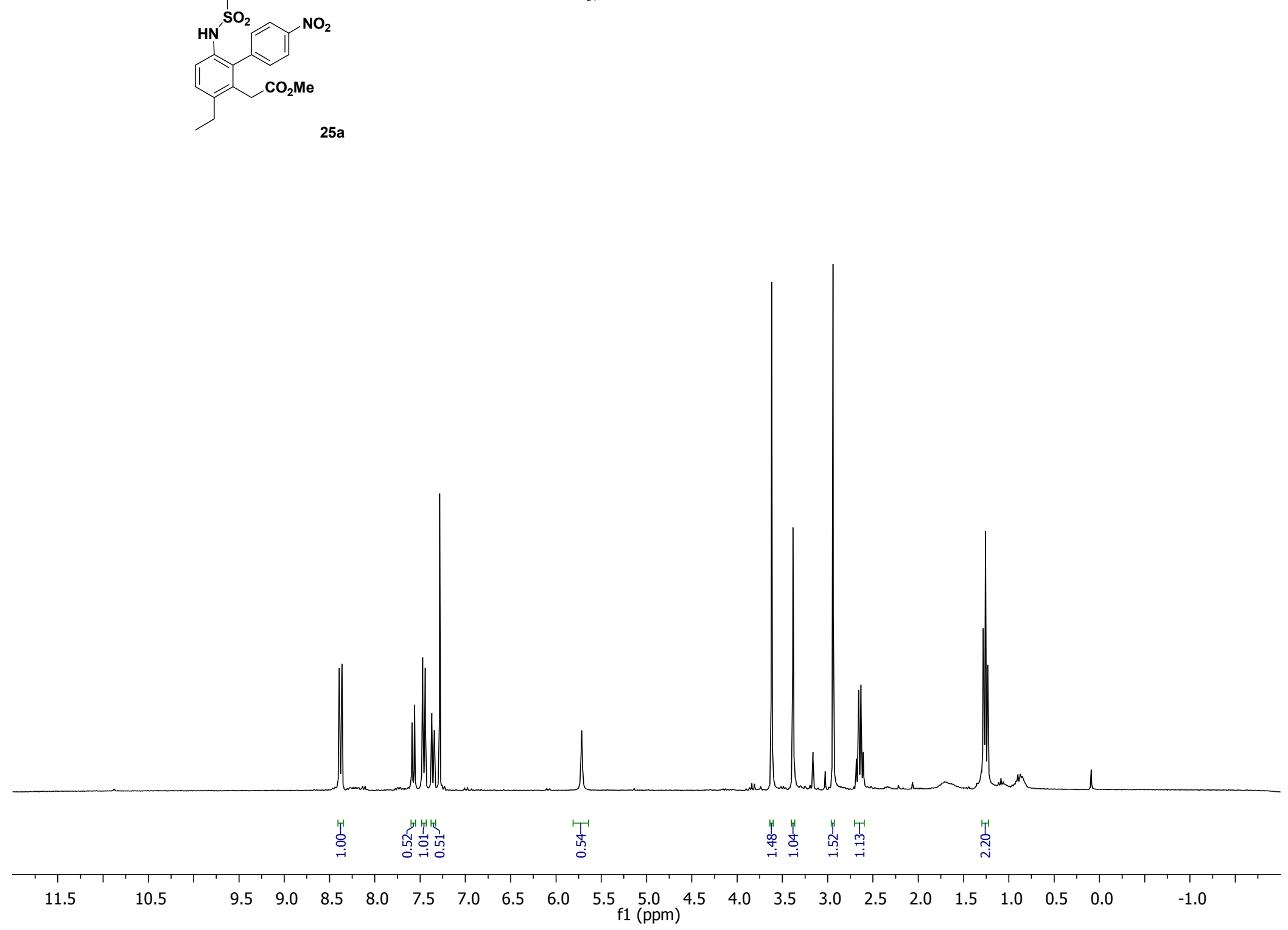
$\mathrm{CDCl}_{3}, 75 \mathrm{MHz}$

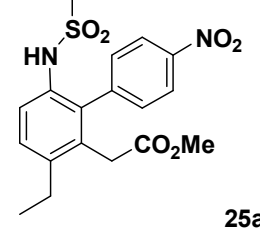

$25 a$

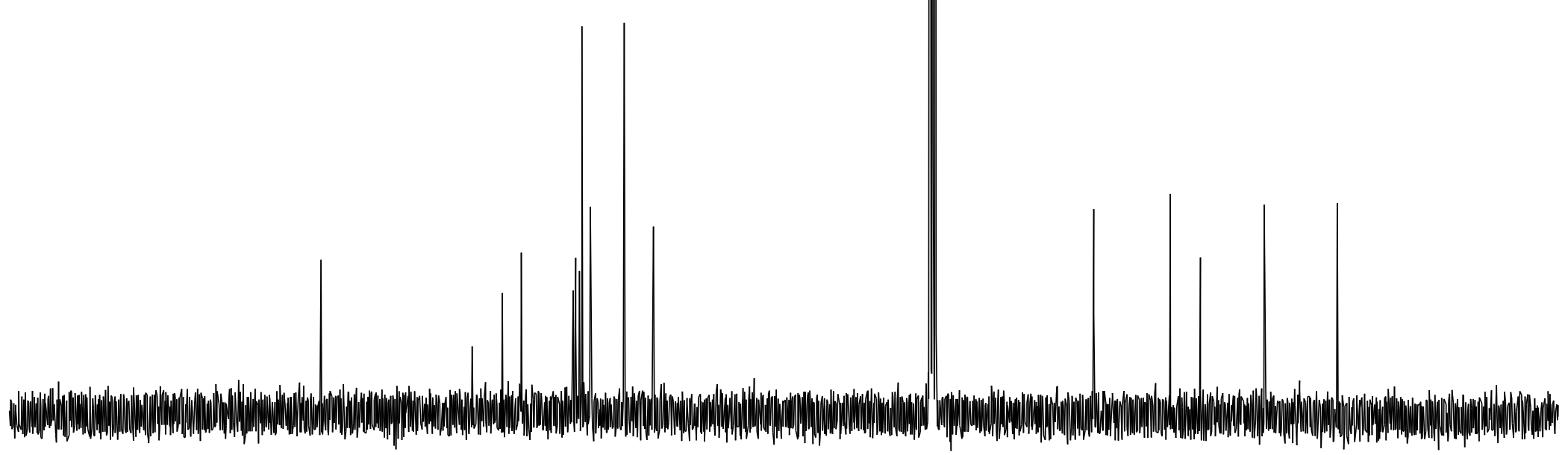

210200 

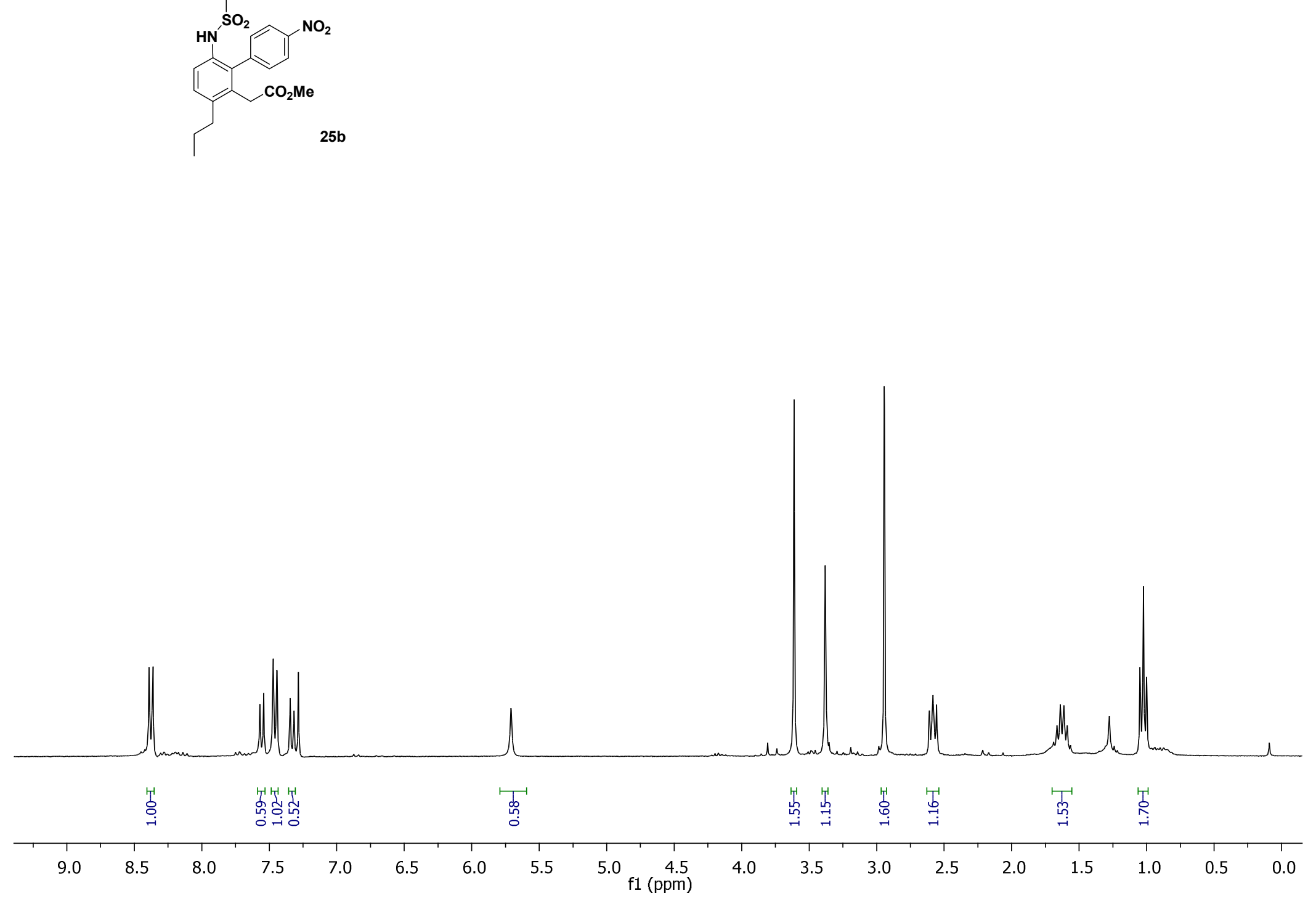


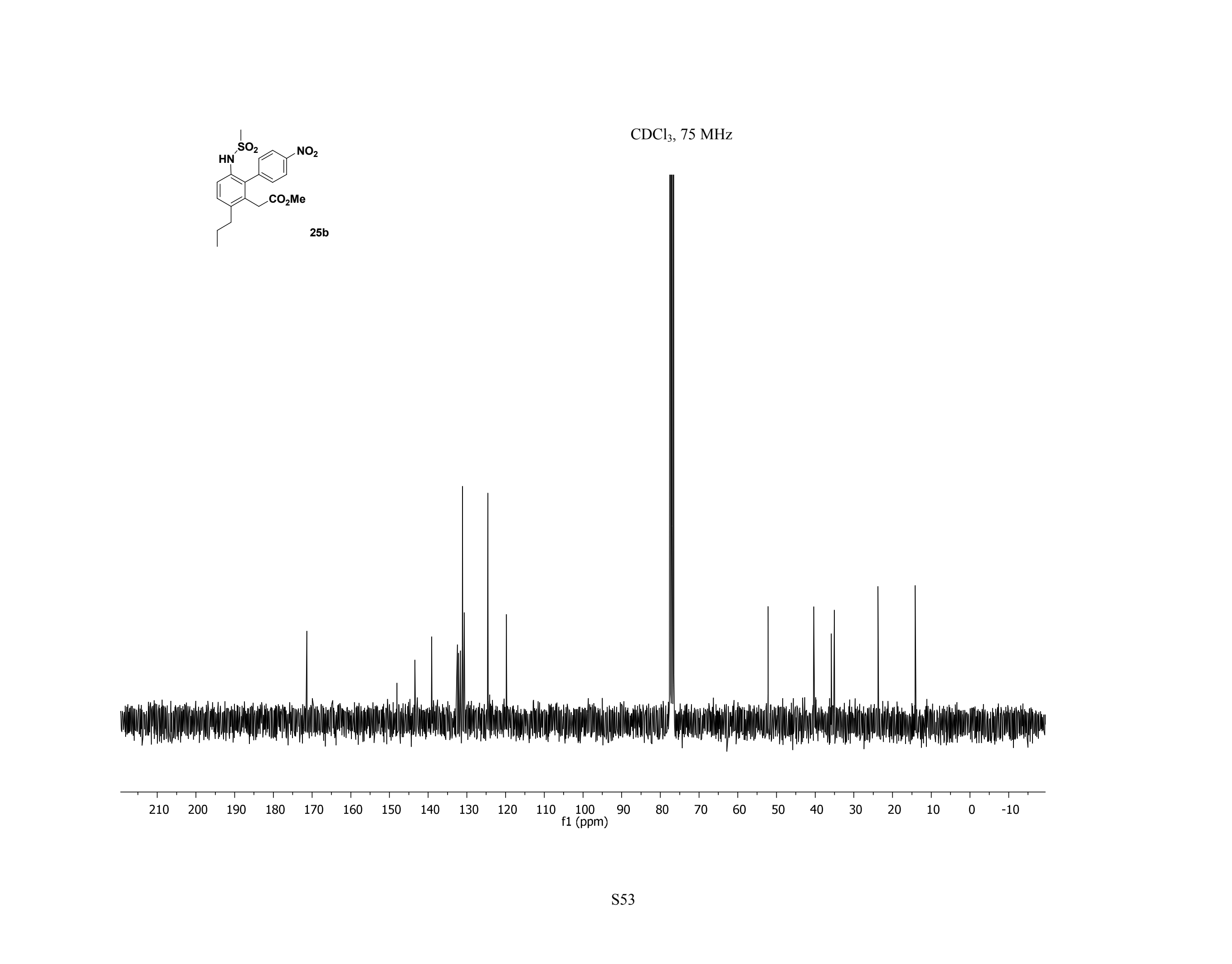






$\mathrm{CDCl}_{3}, 300 \mathrm{MHz}$

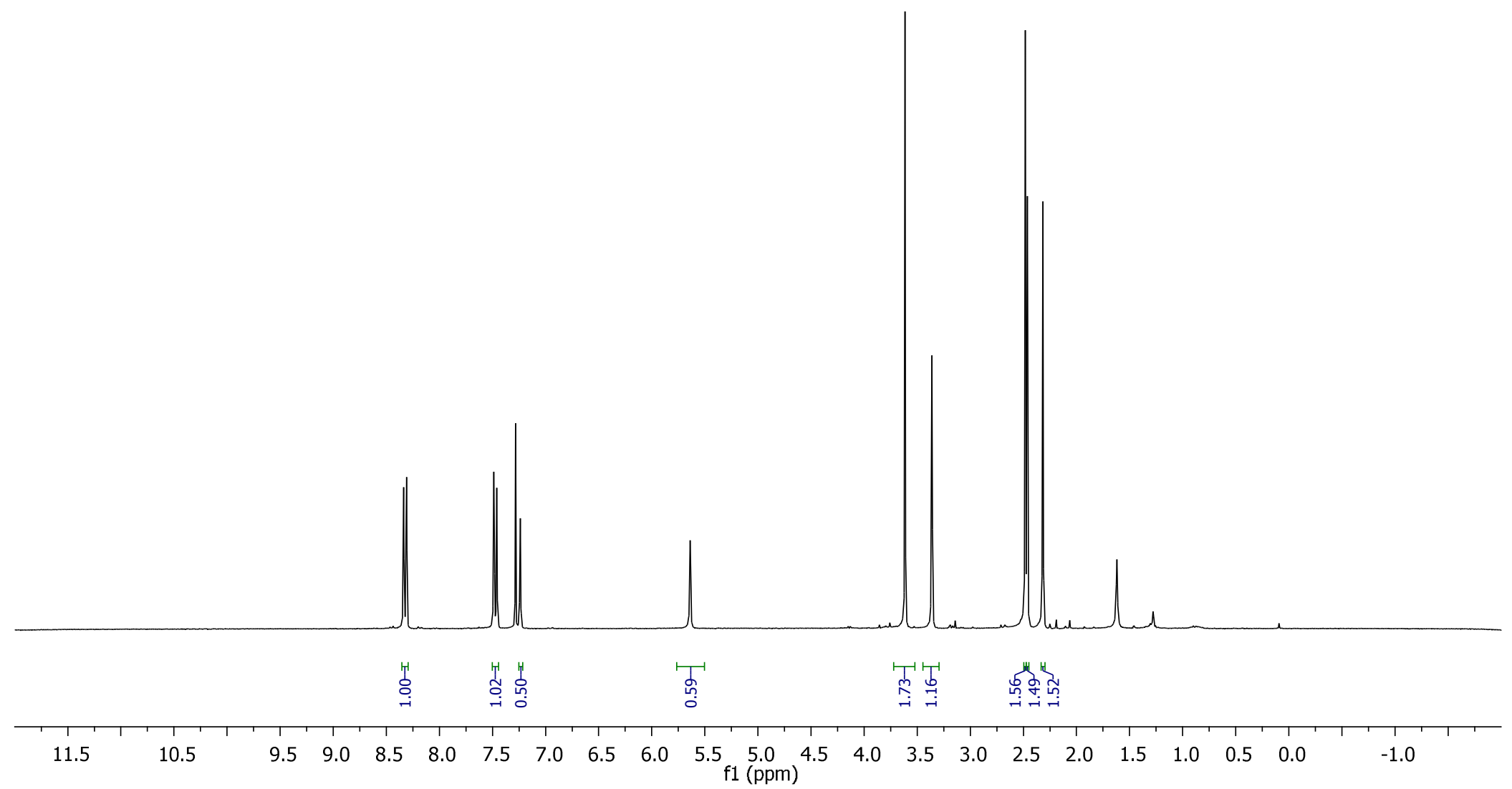




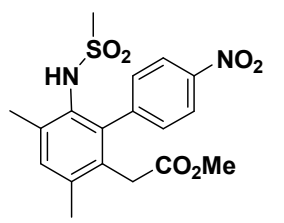

$\mathrm{CDCl}_{3}, 75 \mathrm{MHz}$

25c
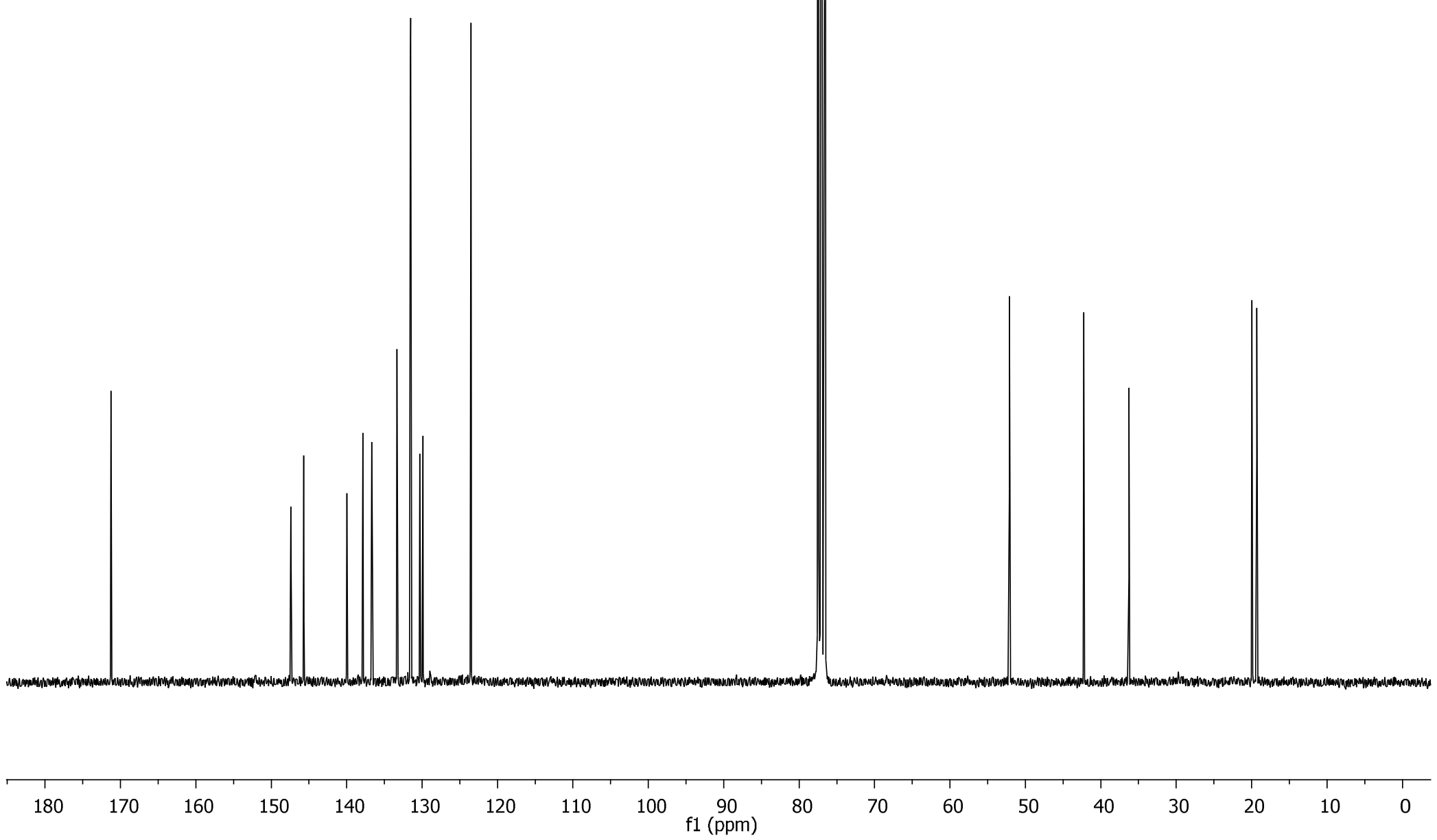

S55 


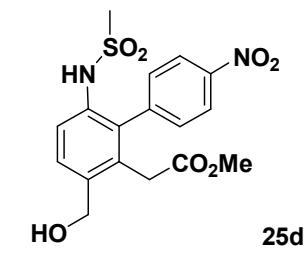

$\mathrm{CDCl}_{3}, 300 \mathrm{MHz}$

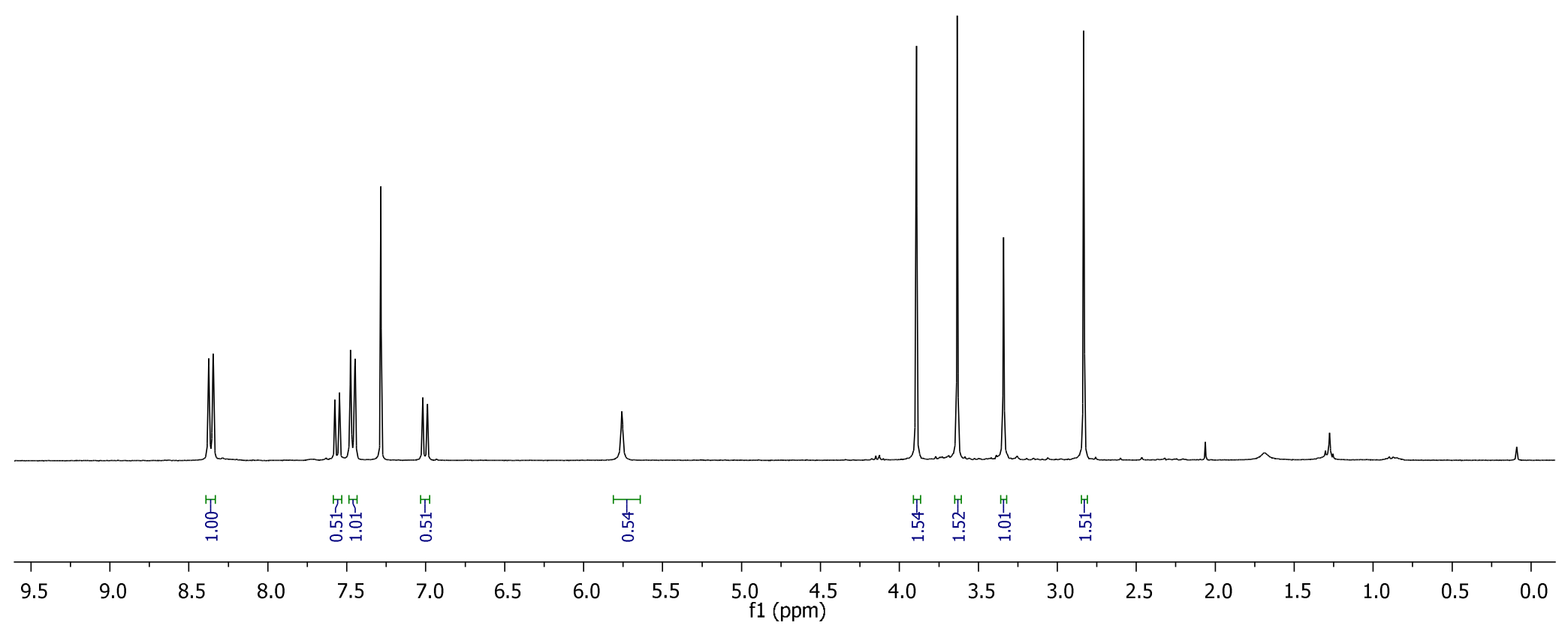




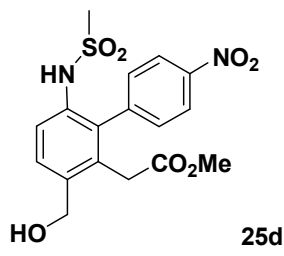

$\mathrm{CDCl}_{3}, 75 \mathrm{MHz}$

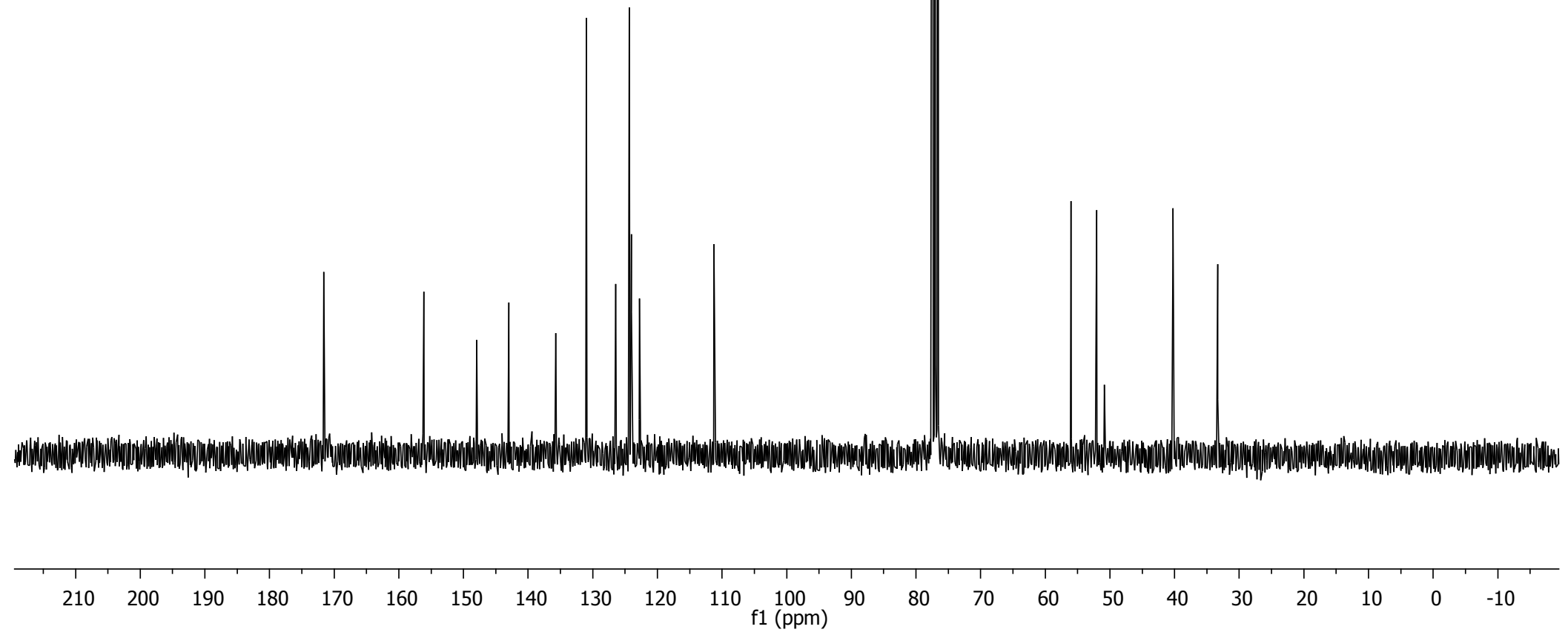




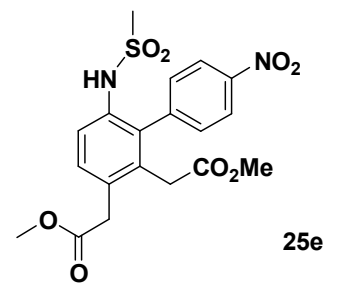

$\mathrm{CDCl}_{3}, 300 \mathrm{MHz}$

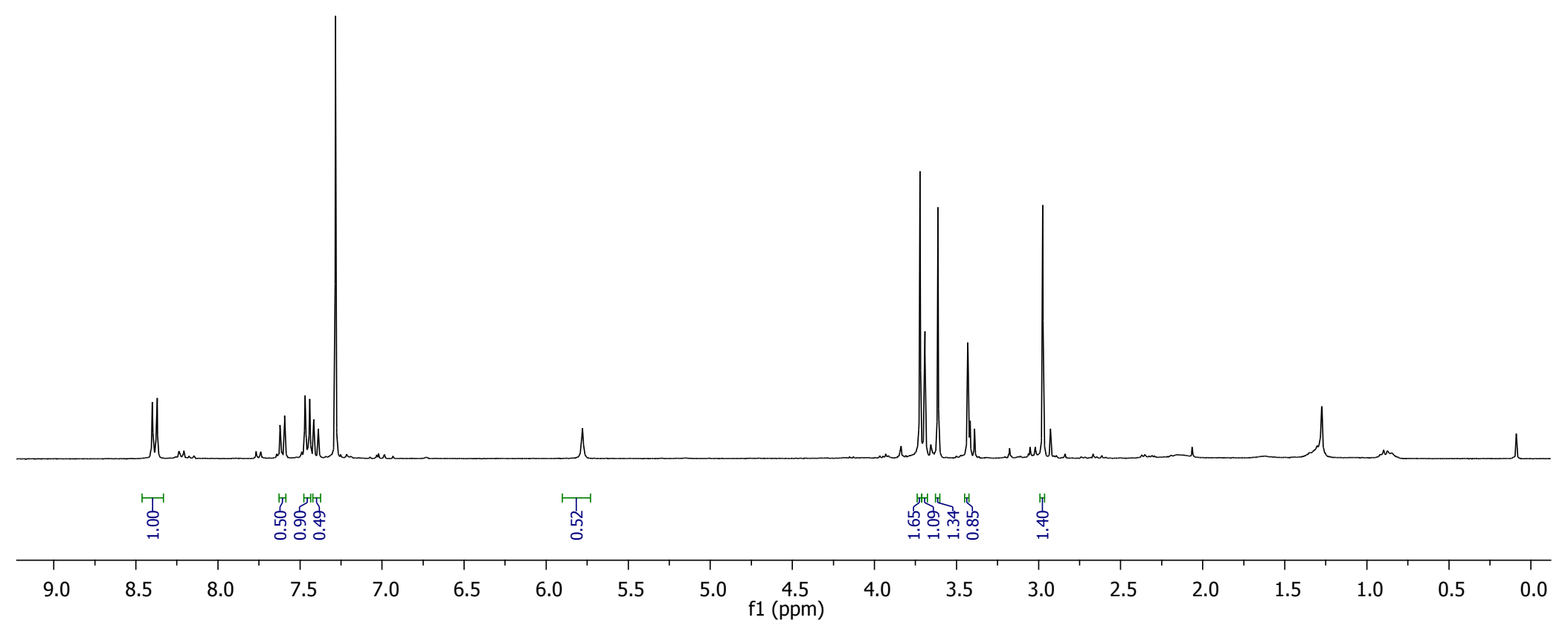


$\mathrm{CDCl}_{3}, 75 \mathrm{MHz}$


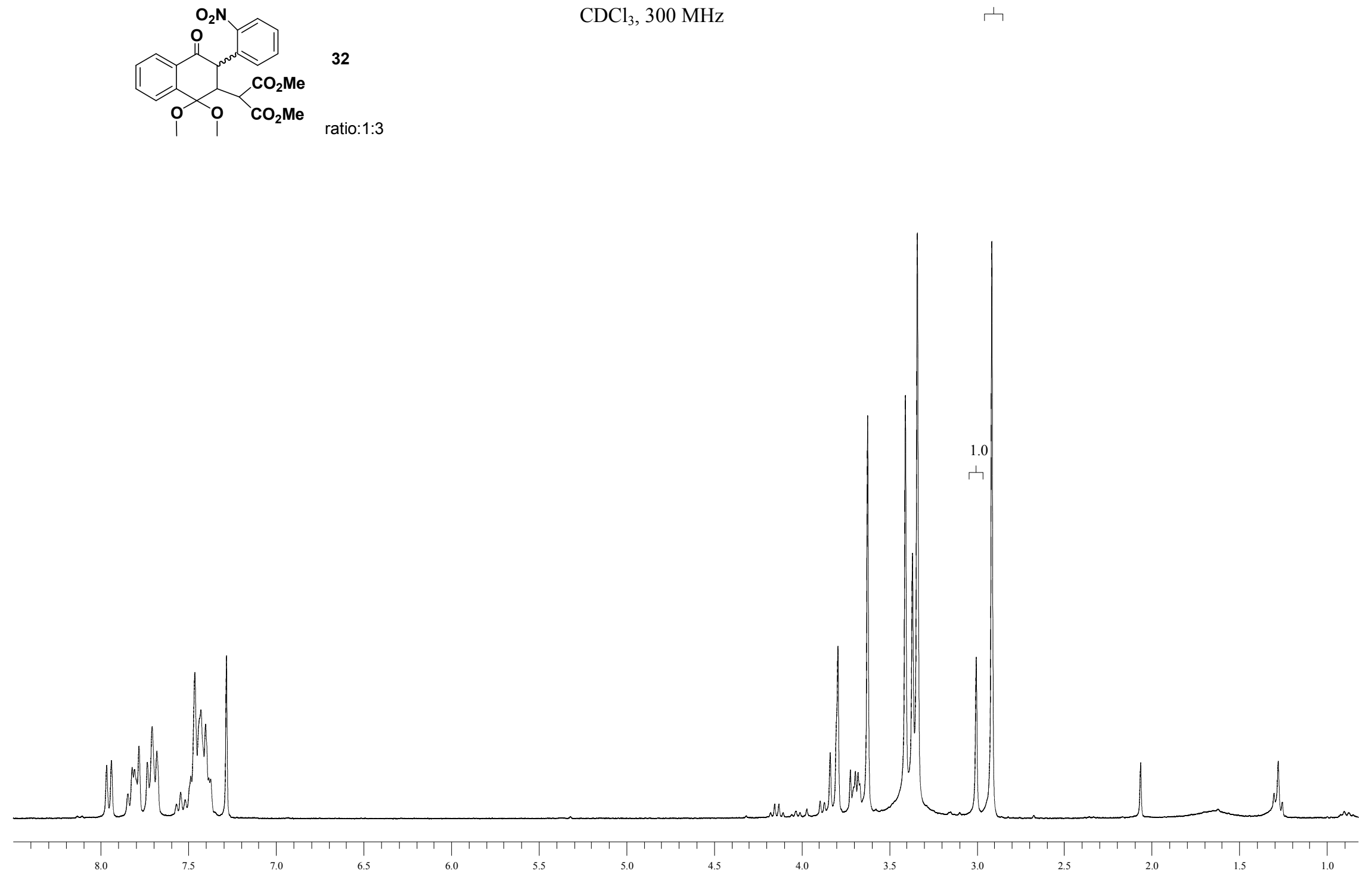


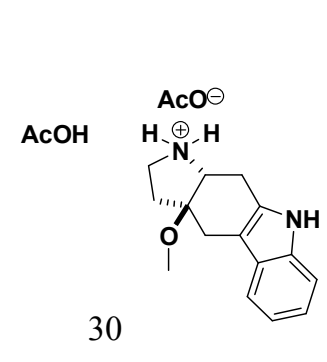

2.2

$\mathrm{CDCl}_{3}, 300 \mathrm{MHz}$

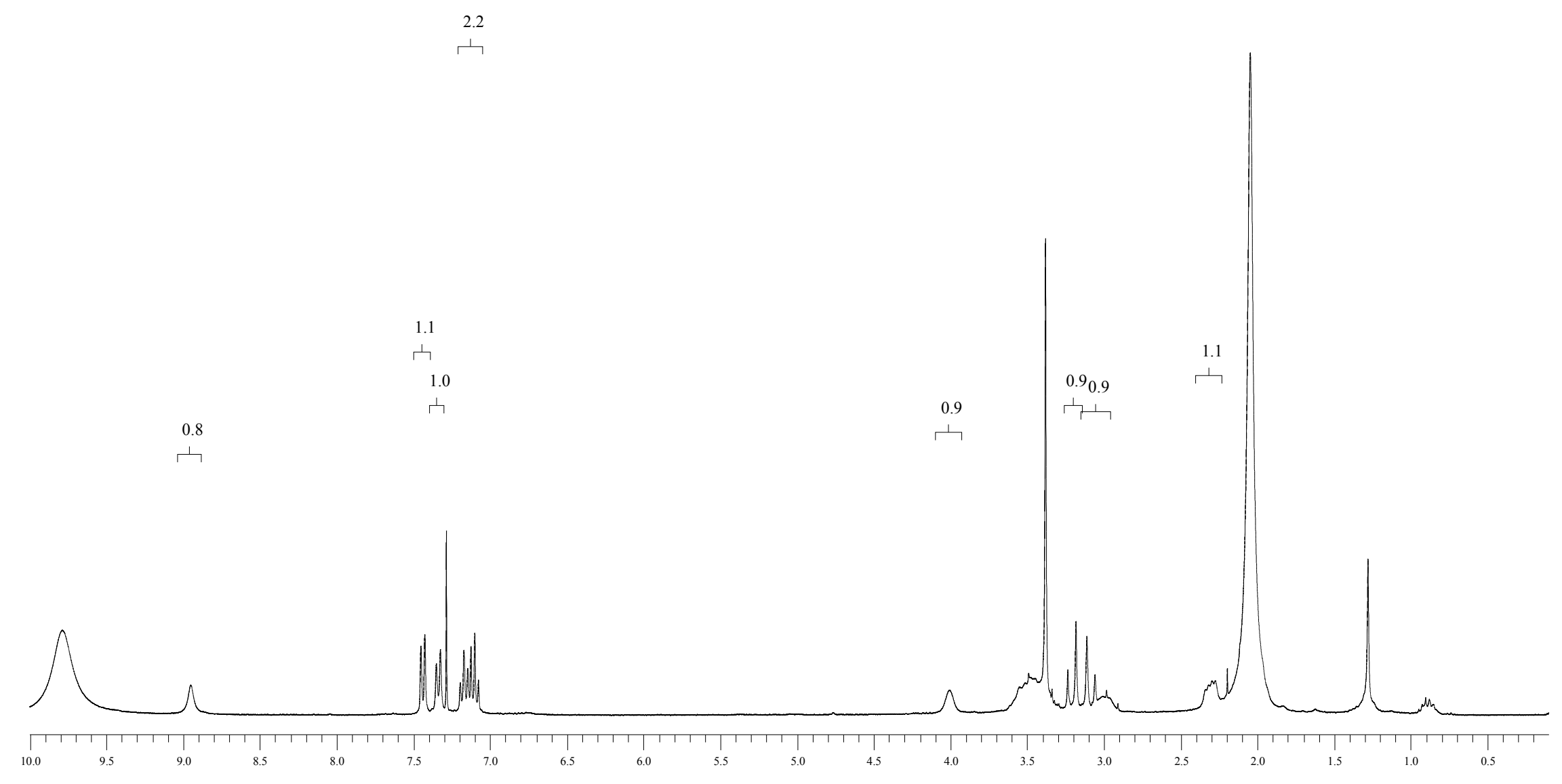




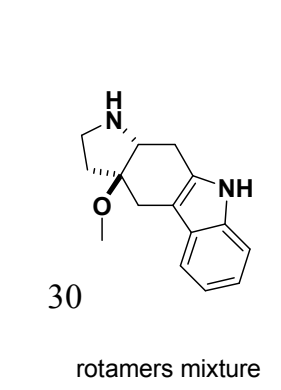

$\mathrm{CDCl}_{3}, 300 \mathrm{MHz}$

5.0

rotamers mixture

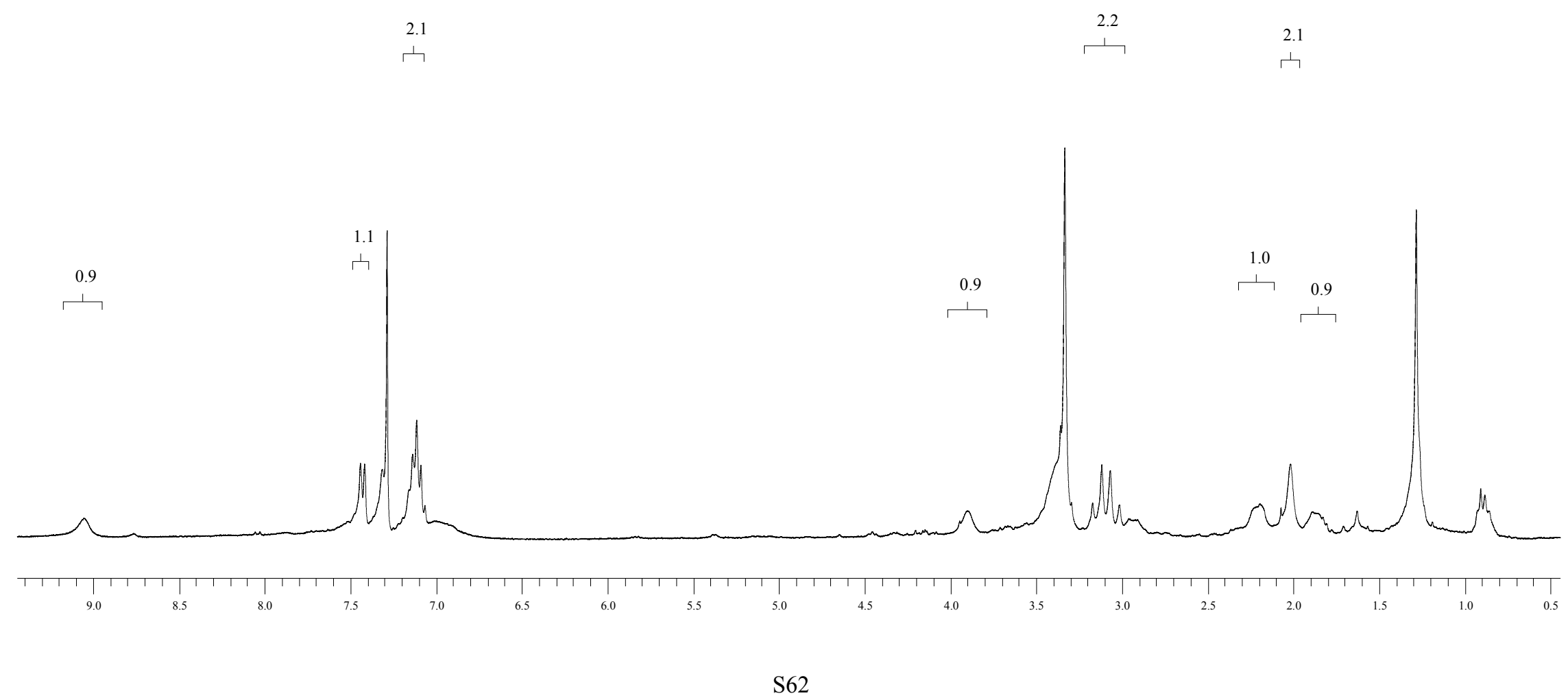


$\mathrm{CDCl}_{3}, 75 \mathrm{MHz}$
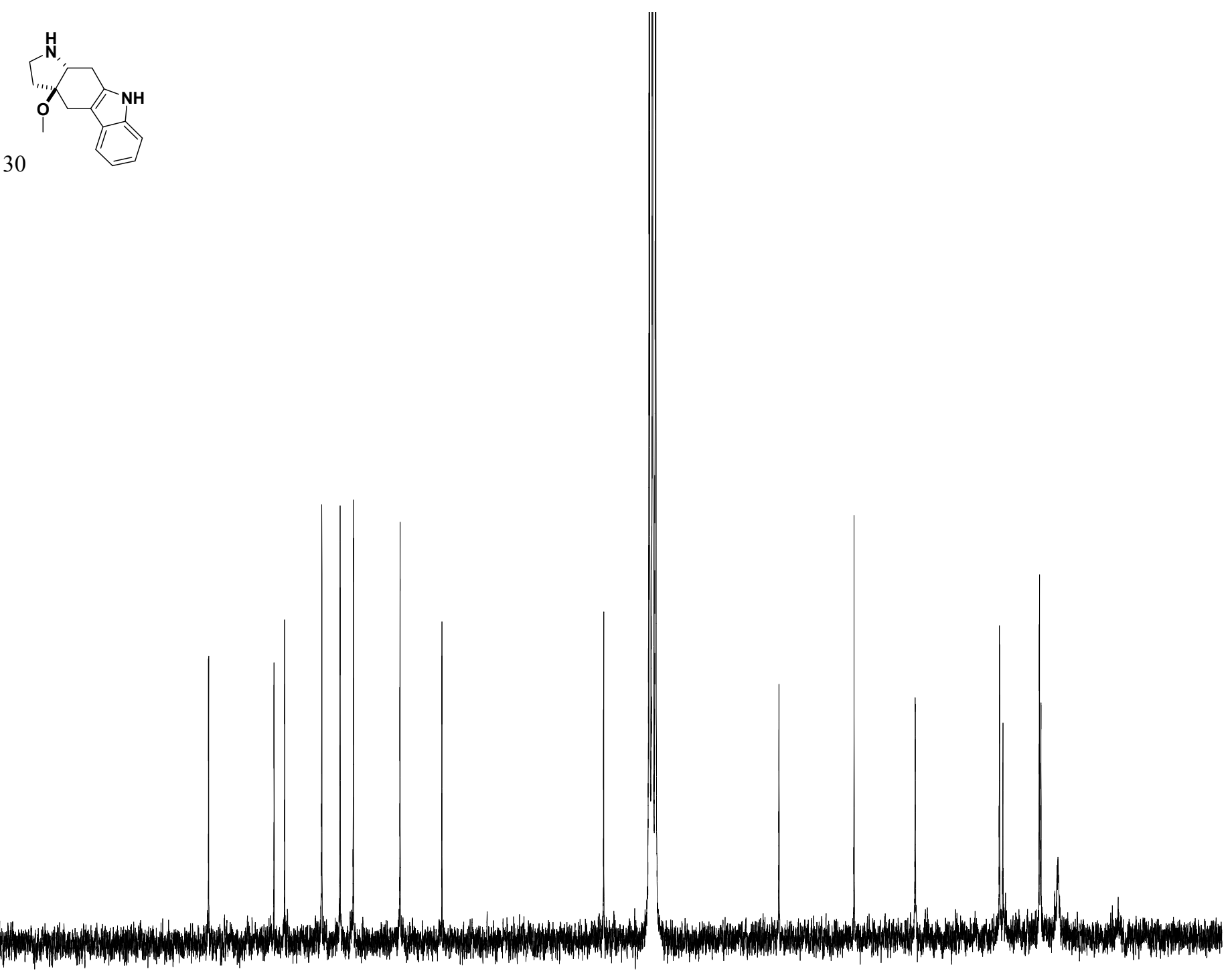

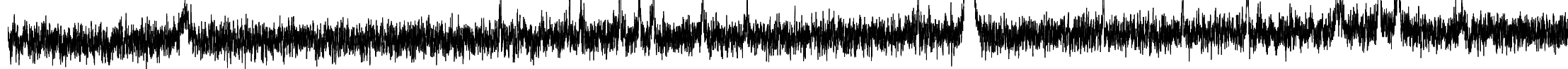

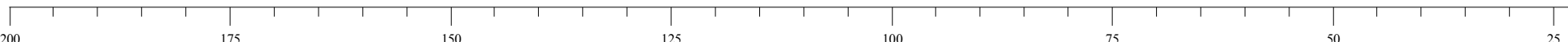




\section{$\mathrm{CDCl}_{3}, 300 \mathrm{MHz}$}

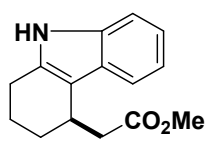

31

2.1

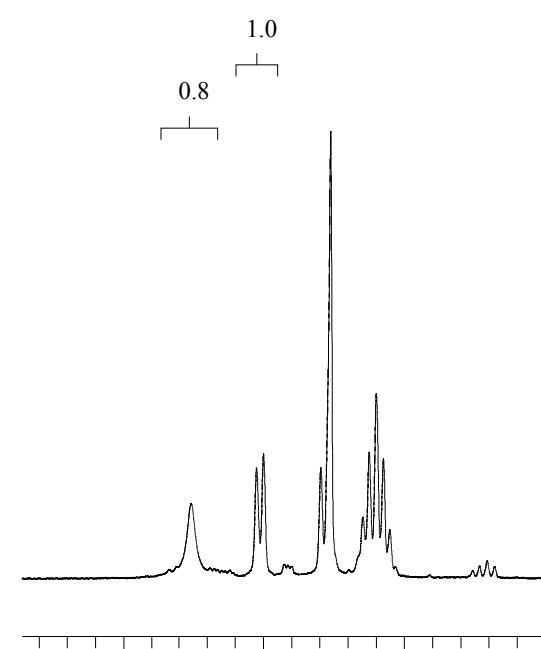


$\mathrm{CDCl}_{3}, 75 \mathrm{MHz}$

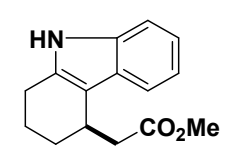

31

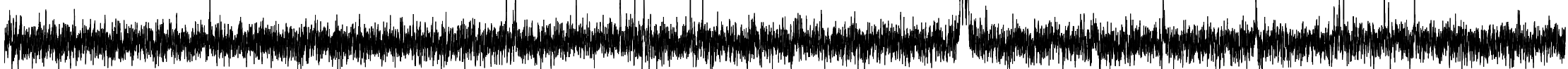


$\mathrm{CDCl}_{3}, 300 \mathrm{MHz}$

$\mathrm{CDCl}_{3}, 75 \mathrm{MHz}$

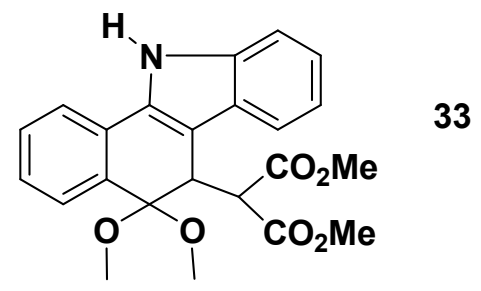

33

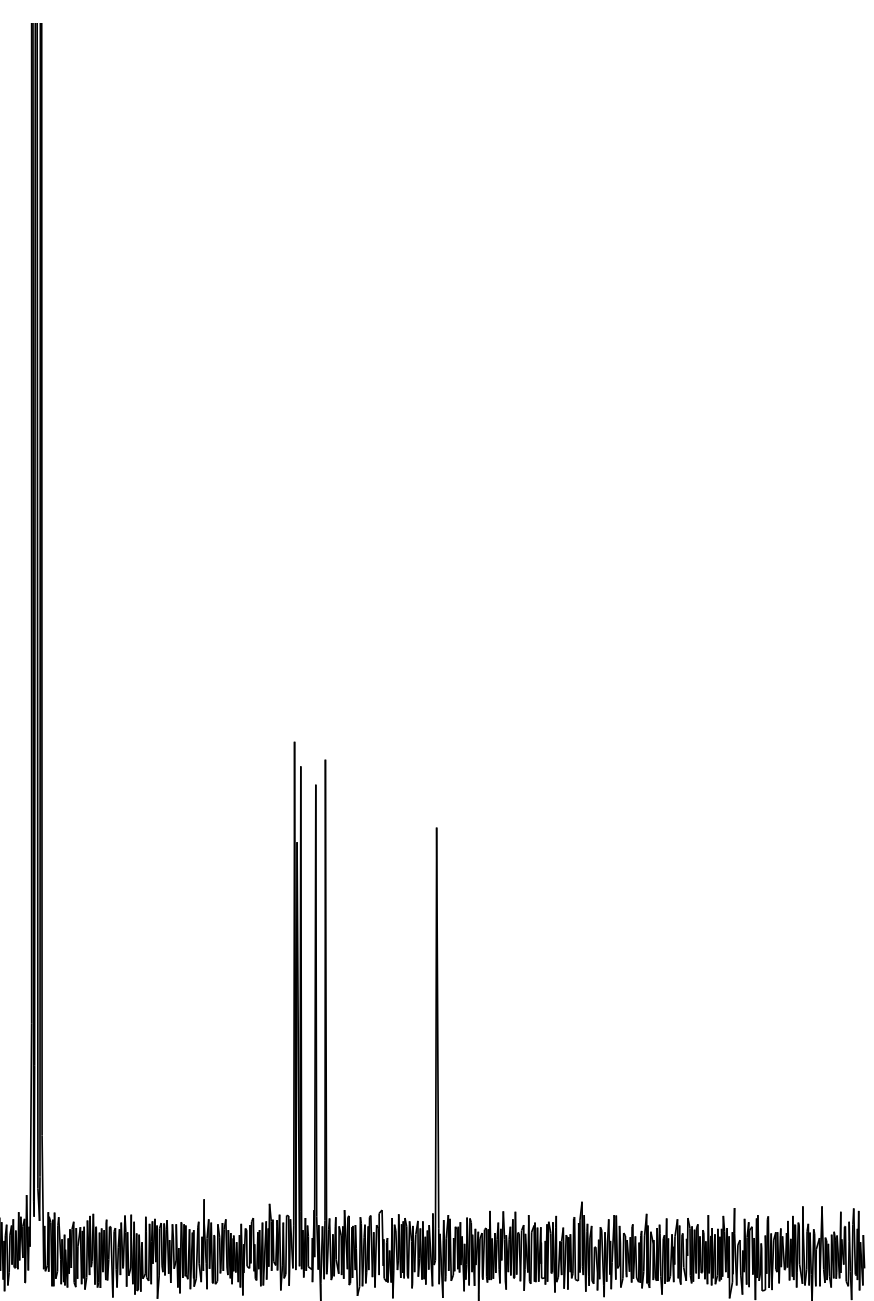

$190 \quad 180$

$150 \quad 140$

130

110

100

80

60

$50 \quad 40$

$\begin{array}{llll}30 & 20 & 10 & 0\end{array}$ 